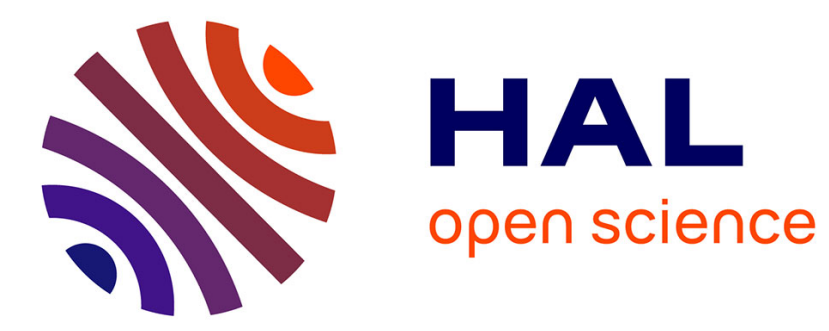

\title{
Large-Eddy Simulations of Turbulence
}

Olivier Métais

\section{To cite this version:}

Olivier Métais. Large-Eddy Simulations of Turbulence. New trends in Turbulence, Springer-Verlag, pp.112-186, 2001, 10.1007/3-540-45674-0_3 . hal-00265309

\section{HAL Id: hal-00265309 \\ https://hal.science/hal-00265309}

Submitted on 19 Feb 2020

HAL is a multi-disciplinary open access archive for the deposit and dissemination of scientific research documents, whether they are published or not. The documents may come from teaching and research institutions in France or abroad, or from public or private research centers.
L'archive ouverte pluridisciplinaire HAL, est destinée au dépôt et à la diffusion de documents scientifiques de niveau recherche, publiés ou non, émanant des établissements d'enseignement et de recherche français ou étrangers, des laboratoires publics ou privés. 


\title{
LARGE-EDDY SIMULATIONS OF TURBULENCE
}

\author{
O. Métais
}

\section{Introduction}

Direct-numerical simulations of turbulence (DNS) consist in solving explicitly all the scales of motion, from the largest $l_{\mathrm{I}}$ to the Kolmogorov dissipative scale $l_{\mathrm{D}}$. It is well known from the statistical theory of turbulence that $l_{\mathrm{I}} / l_{\mathrm{D}}$ scales like $R_{1}^{3 / 4}$, where $R_{\mathrm{l}}$ is the large-scale Reynolds number $u^{\prime} l_{\mathrm{I}} / \nu$ based upon the rms velocity fluctuation $u^{\prime}$. Therefore, the total number of degrees of freedom necessary to represent the whole span of scales of a three-dimensional turbulent flow is of the order of $R_{1}^{9 / 4}$ in three dimensions. In the presence of obstacles, around a wing or a fuselage for instance, and if one wants to simulate three-dimensionally all motions ranging from the viscous thickness $\delta_{\mathrm{v}}=\nu / v_{*} \approx 10^{-6} \mathrm{~m}$ up to $10 \mathrm{~m}$, it would be necessary to put $10^{21}$ modes on the computer. Right now, the calculations done to the expense of not excessive computing times on the biggest machines take about $2 \times 10^{7}$ grid points, which is very far from the above estimation. Even with the unprecedented improvement of scientific computers, it may take several tenths of years (if it becomes ever possible) before DNS permit to simulate situations at Reynolds numbers comparable to those encountered in natural conditions.

Statistical modelling based on Reynolds Averaged Navier-Stokes (RANS) equations are particularly designed to deal with statistically steady flows or with flows whose statistical properties vary "slowly" with time, that is to say of characteristic time scale much larger than a characteristic turbulent time scale. The application of phase averaging constitutes another alternative which allows for the modelling of time periodic flows. With the RANS approach all the turbulent scales are modelled. First order as well as second order RANS models involve many adjustable constants and it is therefore impossible to design models which are "universal" enough to be applicable to various flow configurations submitted to diverse external forces (rotation, thermal stratification, etc.). However, since RANS models compute statistical quantities, they do not require temporal or spatial discretizations as fine as the ones necessary for DNS or even LES. They are therefore applicable to flows in complex geometries. 
Large-Eddy Simulations (LES) techniques constitute intermediate tecniques between DNS and RANS in the sense that the large scales of the flow are deterministically simulated and only the small scales are modelled but statistically influence the large-scale motion. LES then explicitly resolve the large-scales inhomogeneity and anistropy as well as the large-scales unsteadiness. This is important from an engineering point of view since the large scales are responsible for the major part of turbulent transfers of momentum or heat for example. Most subgrid-scale models which parameterized the action of the small-scales are based upon "universal" properties of smallscales turbulence: those can therefore be applied to various flows submitted to various external effects without being modified. In this respect, they constitute "universal" models directly applicable to various flow configurations. However, they require much finer spatial and temporal discretizations than RANS and lie inbetween DNS and RANS as far as CPU time consumption is concerned. Once confined to very simple flow configurations such as isotropic turbulence or periodic flows, the field is evolving to include spatially growing shear flows, separated flows, pipe flows, riblet walls, and bluff bodies, among others. This is due to the tremendous progress in scientific computing and in particular of parallel computing. As will be seen in the few examples presented below, LES are extremely useful in particular towards the understanding of the dynamics of coherent vortices and structures in turbulence. We will show below that this is of special importance for flow control problems, for detached flows and their aeroacoustics predictions and for flows submitted to compressibility effects and density differences.

\subsection{LES and determinism: Unpredictability growth}

From a mathematical viewpoint, the LES problem is not very well posed. Indeed, let us consider the time evolution of the fluid as the motion of a point in a sort of phase space of extremely large dimension (e.g. $\sim 10^{21}$ around a wing, as seen above). At some initial instant, the flow computed with LES will differ from the actual flow, due to the uncertainty contained in the subgridscales. This initial difference between the actual and the computed flow will grow, due to nonlinear effects, as in a dynamical system having a chaotic behaviour. Therefore, the two points will separate in phase space, and, as time goes on, the LES will depart from reality. However, as will be seen below, LES permit to predict the statistical characteristics of turbulence, as well as the dynamics of coherent vortices and structures.

Note that chaos in dynamical systems with a low number of degrees of freedom is generally characterized by a positive Lyapounov exponent, with exponential growth of the distance between two points initially very close in phase space. In isotropic turbulence, one introduces for predictability studies the error spectrum $E_{\Delta}(k, t)$, characterizing the spatial-frequency 
distribution associated to the energy of the difference between two random fields $\vec{u}_{1}$ and $\vec{u}_{2}$ with same statistical properties:

$$
\frac{1}{4}\left\langle\left[\vec{u}_{1}^{2}(\vec{x}, t)-\vec{u}_{2}^{2}(\vec{x}, t)\right]\right\rangle=\int_{0}^{+\infty} E_{\Delta}(k, t) \mathrm{d} k,
$$

the energy spectrum $E(k, t)$ being such that

$$
\frac{1}{2}\left\langle\vec{u}_{1}^{2}\right\rangle=\frac{1}{2}\left\langle\vec{u}_{2}^{2}\right\rangle=\int_{0}^{+\infty} E(k, t) \mathrm{d} k .
$$

The error rate

$$
r(t)=\frac{\int_{0}^{+\infty} E_{\Delta}(k, t) \mathrm{d} k}{\int_{0}^{+\infty} E(k, t) \mathrm{d} k}
$$

is zero when the two fields are completely correlated, and one when they are totally uncorrelated. In predictability studies, one takes generally an initial state such that complete unpredictability $\left(E(k)=E_{\Delta}(k)\right)$ holds above $k_{\mathrm{E}}(0)$, while $E_{\Delta}(k)$ is 0 for $k<k_{\mathrm{E}}(0)$. Two-point closures of the EDQNM type (see [67] for details) show (in three or two dimensions) an inverse cascade of error, where the wavenumber $k_{\mathrm{E}}(t)$ characterizing the error front decreases (see [79]). Thus, the error rate can be approximated by

$$
r(t) \approx \frac{\int_{k_{\mathrm{E}}(t)}^{\infty} E(k, t) \mathrm{d} k}{\int_{0}^{+\infty} E(k, t) \mathrm{d} k} .
$$

We assume that the turbulence is forced by external forces, so that the kinetic energy arising at the denominator of equation (1.3) is fixed. In threedimensional turbulence, and if a $k^{-5 / 3}$ spectrum is assumed for $k>k_{\mathrm{E}}$, the error rate will be proportional to $k_{\mathrm{E}}^{-2 / 3}$. In fact, closures (see $[67,79]$ ) show that $k_{\mathrm{E}}^{-1}$ follows a Richardson's law $\left(k_{\mathrm{E}}^{-1} \propto t^{3 / 2}\right)$, so that the error rate grows linearly with time. This is in fact a slow increase compared with the exponential growth of chaotic dynamical systems, and quite encouraging concerning the potentialities of large-eddy simulations for three-dimensional turbulent flows.

\section{Vortex dynamics}

As will be seen, large-eddy simulations deal with energetic structures of the flow with a characteristic scale or wavelength larger than a given cutoff scale $\Delta x$. These so-called large scales may be spatially organized or not, and sometimes correspond to coherent vortices of recognizable shape. It is therefore important to be able to identify these coherent vortices. 


\subsection{Coherent vortices}

\subsubsection{Definition}

Coherent vortices in turbulence are defined by Lesieur [67] as regions of the flow satisfying three conditions:

(i) the vorticity concentration $\omega$, modulus of the vorticity vector, should be high enough so that a local roll up of the surrounding fluid is possible;

(ii) they should keep approximately their shape during a time $T_{\mathrm{c}}$ longer enough in front of the local turnover time $\omega^{-1}$;

(iii) they should be unpredictable.

In this context, high $\omega$ is a possible candidate for coherent-vortex identification.

\subsubsection{Pressure}

With such a definition, the core of the coherent vortices should be pressure lows. Indeed, a fluid parcel winding around the vortex will be (in a frame moving with the parcel) in approximate balance between centrifugal and pressure-gradient effects. We are talking here of the static pressure $p$. The reasoning may be made more quantitative by considering Euler equation (in a flow of uniform density $\rho_{0}$ ) in the form

$$
\frac{\partial \vec{u}}{\partial t}+\vec{\omega} \times \vec{u}=-\frac{1}{\rho_{0}} \vec{\nabla} P
$$

where $P=p+\rho_{0} \vec{u}^{2} / 2$ is now the dynamic pressure. In a frame moving with the coherent vortex and supposed locally Galilean, the ratio of the second to the first term in the l.h.s. of equation (2.1) is of the order of $T_{\mathrm{c}} \omega$. Then the equation reduces to the cyclostrophic balance

$$
\vec{\omega} \times \vec{u} \approx-\frac{1}{\rho_{0}} \vec{\nabla} P
$$

if condition (ii) above is fulfilled. If one supposes that the coherent vortex is a vortex tube tangent to the velocity vector, it follows that this tube is a low for the dynamic pressure.

\subsubsection{The $Q$-criterion}

We recall now the so-called $Q$-criterion. Let

$$
S_{i j}=\frac{1}{2}\left(\frac{\partial u_{i}}{\partial x_{j}}+\frac{\partial u_{j}}{\partial x_{i}}\right), \quad \Omega_{i j}=\frac{1}{2}\left(\frac{\partial u_{i}}{\partial x_{j}}-\frac{\partial u_{j}}{\partial x_{i}}\right)
$$


be respectively the symmetric and antisymmetric parts of the velocitygradient tensor $\partial u_{i} / \partial x_{j}$. It is well known that the second invariant of this tensor

$$
Q=\frac{1}{2}\left(\Omega_{i j} \Omega_{i j}-S_{i j} S_{i j}\right)=\frac{1}{4}\left(\vec{\omega}^{2}-2 S_{i j} S_{i j}\right),
$$

is equal to $\nabla^{2} p / 2$. Indeed, the Poisson equation for the pressure in a flow of uniform density writes

$$
\begin{aligned}
-\frac{1}{\rho_{0}} \nabla^{2} p & =\frac{\partial^{2}}{\partial x_{i} \partial x_{j}} u_{i} u_{j}=\frac{\partial}{\partial x_{i}}\left[u_{j} \frac{\partial u_{i}}{\partial x_{j}}\right]=\frac{\partial u_{i}}{\partial x_{j}} \frac{\partial u_{j}}{\partial x_{i}} \\
& =\left(S_{i j}+\frac{1}{2} \epsilon_{i j \lambda} \omega_{\lambda}\right)\left(S_{j i}+\frac{1}{2} \epsilon_{j i \mu} \omega_{\mu}\right)=S_{i j} S_{i j}-\frac{1}{2} \vec{\omega}^{2}=-2 Q .
\end{aligned}
$$

Let us consider a low-pressure tube of small section. Let $\Delta \Sigma$ be its lateral surface, assumed isobaric and convex. Let $\Sigma_{1}$ and $\Sigma_{2}$ be two cross sections of the tube, supposed normal to its axis, and $\Delta V$ the volume of the tube portion comprised between $\Sigma_{1}$ and $\Sigma_{2}$. The pressure gradient on $\Delta \Sigma$ is normal to it and directed towards the exterior. The pressure gradient on the two cross sections is tangent to them. Then, the flux of the pressure gradient getting out of the tube is equal to the flux through $\Delta \Sigma$, and is positive. Due to the divergence theorem, this is equal to the integral over $\Delta V$ of $\nabla^{2} p$, which is positive, such as the integral of $Q$. If we suppose that the size of $\Delta V$ is small enough so that $Q$ does not vary appreciably within it, this implies that $Q$ is positive in $\Delta V$. Since this reasoning may be repeated all along the length of the tube, the $Q$-criterion $(Q>0)$ is therefore a necessary condition for the existence of such thin convex lowpressure tubes. This is the reason which motivates Hunt et al. [46] to propose the $Q$-criterion as a way to characterize the vortices.

We have thus shown that the $Q$-criterion is valuable to help characterizing the convex low-pressure tubes, which are generally associated to coherent vortices. Notice however that the relation $Q=\nabla^{2} p / 2 \rho_{0}$ implies that vortex-identification criteria based upon $Q$ involve much more smallscale activity than thosed based on the pressure, as will be verified in the simulations.

\subsection{Vortex identification}

Let us present a comparison of some of these vortex-identification methods (low dynamic pressure, high $\omega$, positive $Q$ ) applied to incompressible DNS of isotropic turbulence and LES of a backward-facing step, done by Delcayre [28]. Other exemples will be provided in the rest of the chapter. More specifically, we consider isosurfaces at a given threshold of $\omega, p$ and $Q$. The 


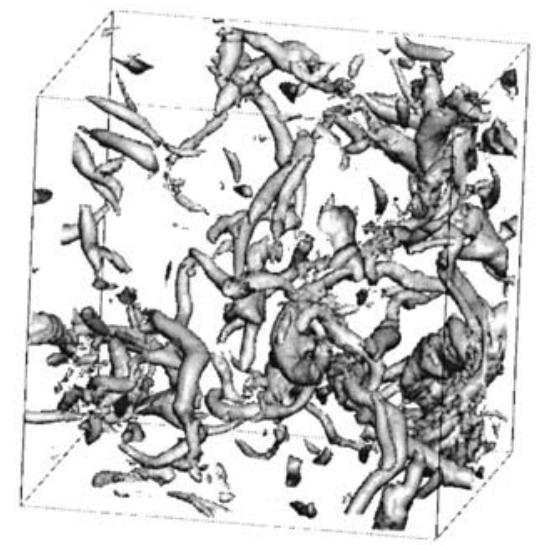

Fig. 1. Low-pressure isosurfaces in DNS of isotropic turbulence (from Delcayre $[28])$.
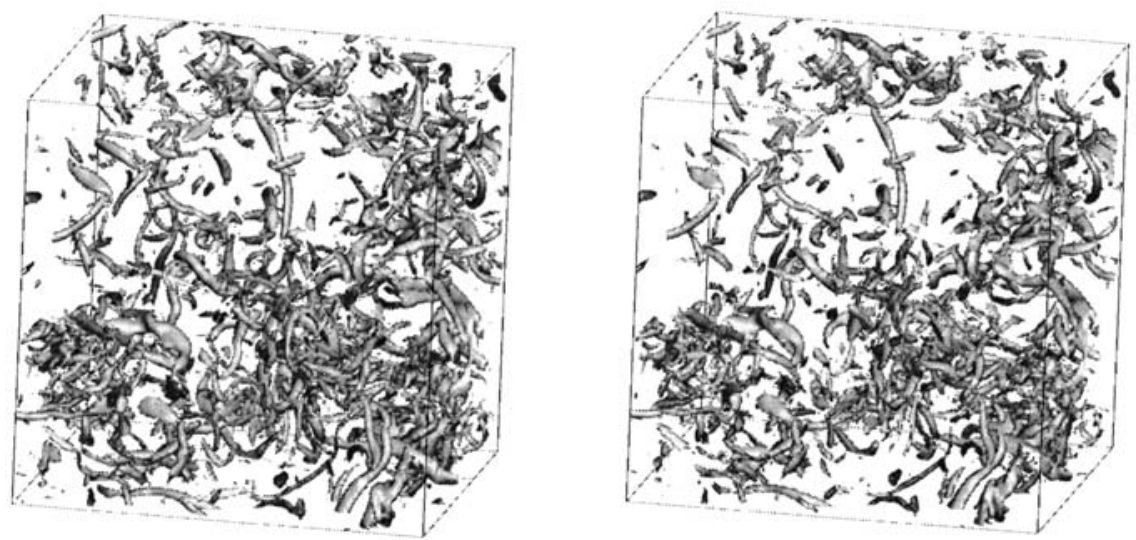

Fig. 2. High vorticity (left) and positive $Q$ (right) isosurfaces in DNS of isotropic turbulence (from Delcayre [28]).

choice of the threshold is justified by what gives visually the best vortices, or with respect to what we know of the flow dynamics from former simulations or laboratory experiments.

\subsubsection{Isotropic turbulence}

For isotropic turbulence, we consider a DNS at low Reynolds number (freelydecaying case). It is well known that coherent vortices exist in such a flow, in 
the form of thin tubes randomly orientated, of length the turbulence integral scale (Siggia [102], Vincent and Ménéguzzi [111], Métais and Lesieur [80], Jimenez and Wray [47]). Comparison of Figures 1 and 2 (left) show that the isobaric surfaces are more fat than the vorticity surfaces, but represent the same events, in good agreement with the observations of Brachet [13] for Taylor-Green vortices and Métais and Lesieur [80] for LES of isotropic turbulence. This is confirmed by the present DNS. Figure 2 (right), showing the iso- $Q$ maps, is close to the vorticity map, althought slightly less dense. A last point concerns the dimension of these tubes: everybody agrees on the average length, which is integral scale $l$. It is right now not decided yet whether the diameter scales on the dissipative scale or the Taylor microscale. If we interpret the vortices as resulting from the roll up of local vortex sheets, it is this last scale which should prevail. As a matter of fact, this strongly anisotropic vortex topology is very far from the quite naive circular eddies considered in the popular folklore of Richardson-Kolmogorov cascade.

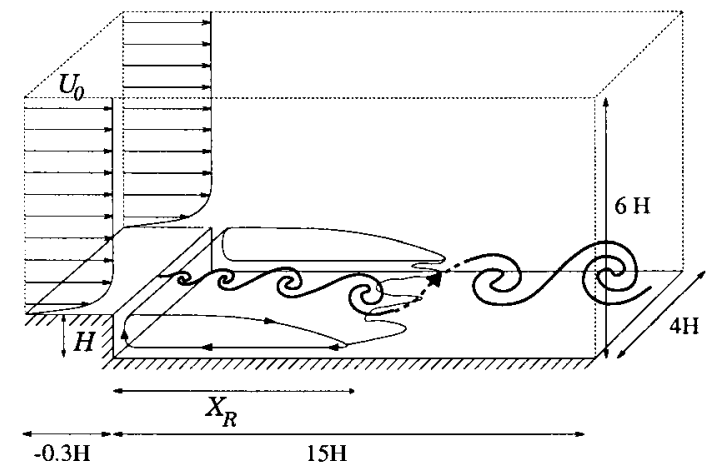

Fig. 3. Schematic view of the backward-facing step (from Delcayre [28]).

\subsubsection{Backward-facing step}

We present now LES results of a uniform-density flow above a straight backward-facing step. The model used is the selective structure-function model (SSF, see Sect. 5.2). Figure 3 shows a schematic view of the flow. The step-height is $H$, the expansion ratio 1.2, and the Reynolds number $U_{0} H / \nu=5100$, as in the configuration studied experimentally (Jovic and Driver [49]) and numerically (Le et al. [62], Akselvoll and Moin [2]) at Stanford. A free-slip boundary condition is used on the upper boundary, well justified with respect to the laboratory experiment consisting in a double-expansion channel. At the inlet, we impose Spalart's [106] mean turbulent boundary-layer velocity profile, to which a small three-dimensional 
white-noise perturbation regenerated at each time step is superposed. One assumes periodicity in the spanwise direction, and there is an outflow boundary condition of the Sommerfeld type, where the quantities are transported following a fictitious "tangential" wave phase velocity. We have checked that the latter is very good for letting the coherent vortices get out of the computational domain without any distorsion.
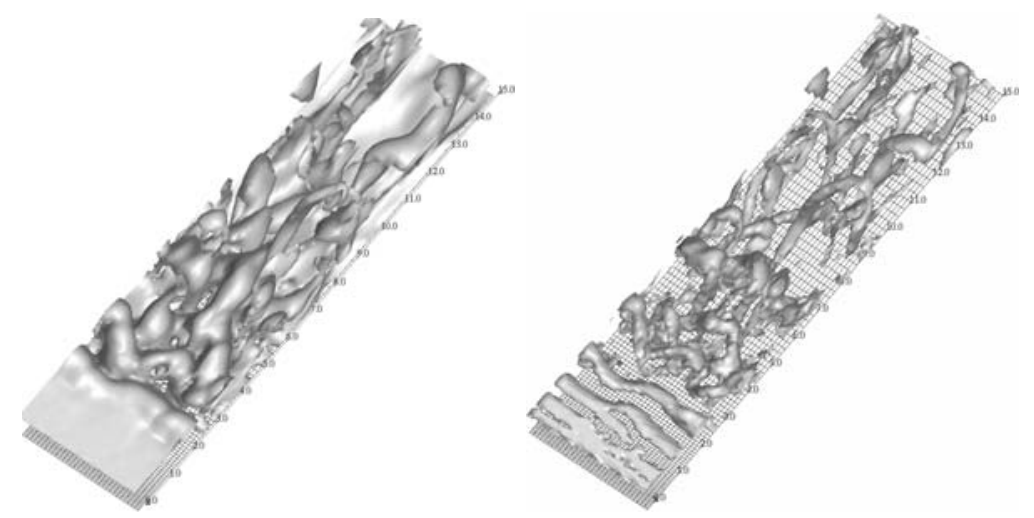

Fig. 4. Backward-facing step, visualization of coherent vortices using highvorticity modulus (left) and positive $Q$ (right) isosurfaces (from Delcayre [28]).

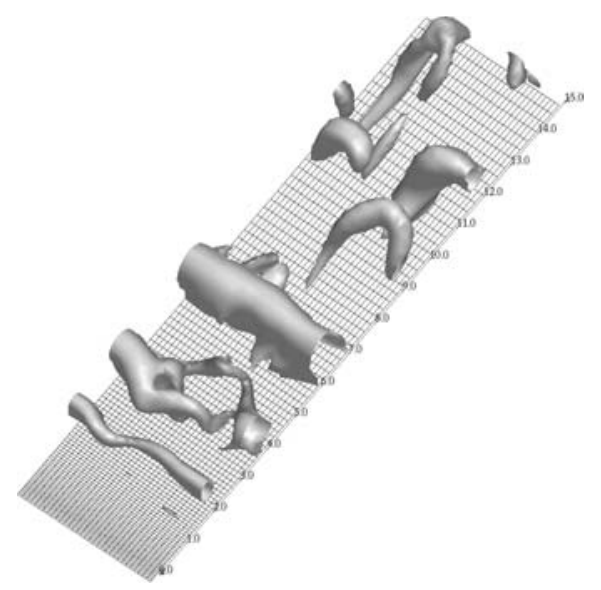

Fig. 5. Backward-facing step, low-pressure isosurfaces (from Delcayre [28]).

Flow animations show the following vortex dynamics: quasi twodimensional Kelvin-Helmholtz type vortices are shed behind the step, 
resulting from the instability of the upstream vortex sheet. Then they are subject to dislocations (helical pairings) and transform into a field of big staggered arch-like vortices which impact the lower wall and are carried away downstream. Figure 4 (left), presenting iso-vorticity maps, does show the breakdown of the vortex sheet into big staggered $\Lambda$-vortices. Figure 4 (right) presents iso- $Q$ maps, and indicates the same vortex events as for the vorticity, but the vortices are thinner, and the upstream vortex sheet has been erased. Finally, isobaric surfaces (Fig. 5) are misleading in this case, since they seem to indicate a large quasi two-dimensional vortex at the level of reattachment, whereas it is simply an erroneous reconnection of the tips of the big $\Lambda$ 's.

Such vortical structure has important implications in terms of pressure spectra and aeroacoustics. Figure 6 indeed shows the frequency pressure spectra at four positions in the flow: (1) just behind the step, (2) just before reattachment, (3) just behind reattachment and (4) much further downstream. Frequencies $f$ are expressed in units $U_{0} / H$ and correspond in fact to Strouhal numbers $S_{\mathrm{t}}=f H / U_{0}$. Position (1) is marked by a peak at $S_{\mathrm{t}}=0.23$, corresponding to the shedding of Kelvin-Helmholtz vortices. At position (2), a second peak of higher amplitude is present at the subharmonic Strouhal number 0.12, corresponding physically to helical pairing. At positions (3) and (4), the two previous Strouhal numbers are still there, but a third peak forms at a Strouhal $S_{\mathrm{t}}=0.07$, corresponding to the well-known flapping of the recirculation bubble. These different Strouhal numbers associated with the different unsteady phenomena are in good agreement with those previously found by other authors (see e.g. Le et al. [62], Arnal and Friedrich [6]). Such informations regarding the pressure spectra and how they relate to the vortex dynamics is very important for acoustical studies and noise control in particular.

\section{LES formalism in physical space}

This chapter deals with an incompressible flow, whose density is conserved with the fluid motion, which implies the continuity equation $\vec{\nabla} \vec{u}=0$. Then $\rho$ may be either uniform, or have a mean variation taken into account through Boussinesq's approximation.

\subsection{LES equations for a flow of constant density}

To begin with, let us consider a simulation of Navier-Stokes equations with constant density $\rho_{0}$ carried out in physical space, using finite-difference or finite-volume methods. Let $\Delta x$ be a scale characteristic of the grid mesh. In order to eliminate the subgridscales, we introduce a filter of width $\Delta x$. 

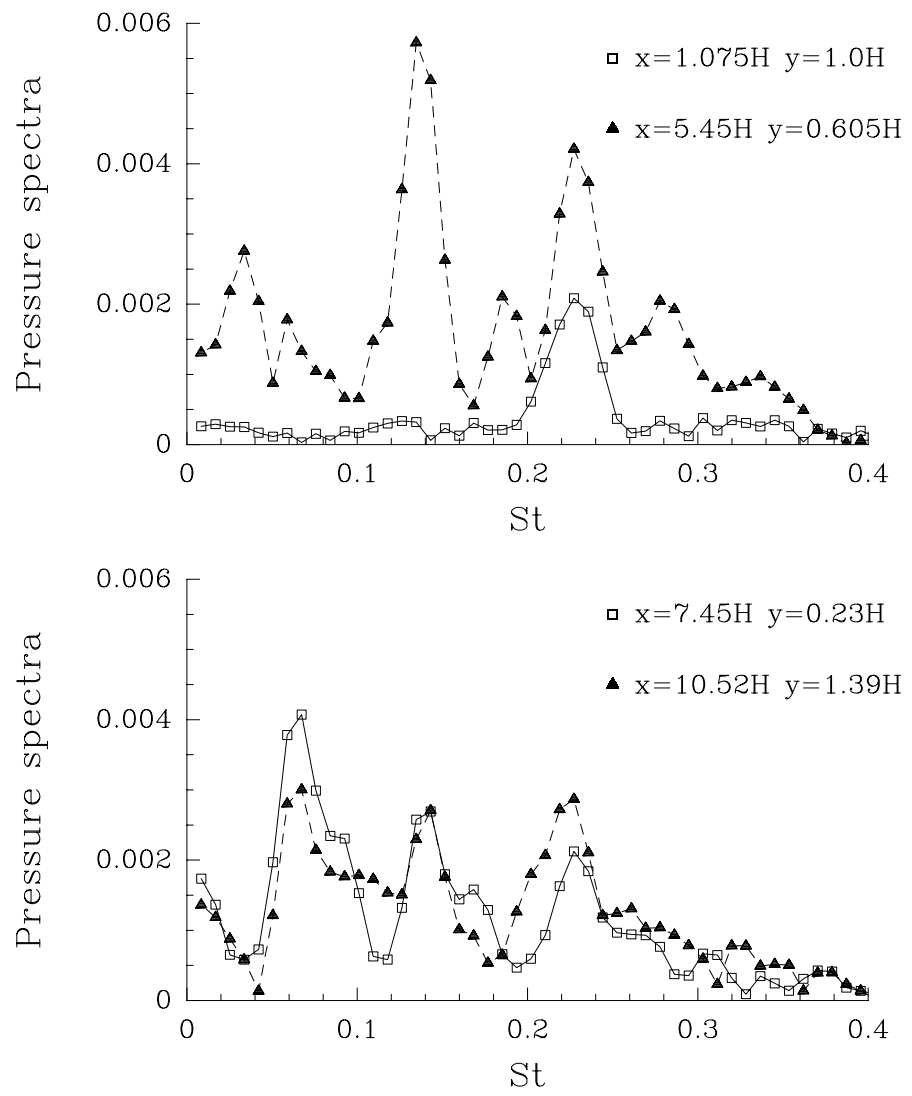

Fig. 6. Backward-facing step, spectra of spanwise-averaged pressure fluctuations at different positions (courtesy Delcayre).

Mathematically, the filtering operation corresponds to the convolution of any quantity $f(\vec{x}, t)$ of the flow by the filter function $G_{\Delta x}(\vec{x})$, in the form

$$
\bar{f}(\vec{x}, t)=\int f(\vec{y}, t) G_{\Delta x}(\vec{x}-\vec{y}) \mathrm{d} \vec{y}=\int f(\vec{x}-\vec{y}, t) G_{\Delta x}(\vec{y}) \mathrm{d} \vec{y},
$$

and the subgridscale field is the departure of the actual flow with respect to the filtered field:

$$
f=\bar{f}+f^{\prime} .
$$

Since $\Delta x$ is here assumed constant, it is easy to show that the space and time derivatives commute with the filtering operator. 
We assume that we use a Cartesian system or coordinates. Let us first write themomentum equations as

$$
\frac{\partial u_{i}}{\partial t}+\frac{\partial}{\partial x_{j}}\left(u_{i} u_{j}\right)=-\frac{1}{\rho_{0}} \frac{\partial p}{\partial x_{i}}+\frac{\partial}{\partial x_{j}}\left(2 \nu S_{i j}\right),
$$

where $S_{i j}$ is the deformation tensor already defined by equation (2.3). The filtered momentum equations write exactly

$$
\frac{\partial \bar{u}_{i}}{\partial t}+\frac{\partial}{\partial x_{j}}\left(\bar{u}_{i} \bar{u}_{j}\right)=-\frac{1}{\rho_{0}} \frac{\partial \bar{p}}{\partial x_{i}}+\frac{\partial}{\partial x_{j}}\left(2 \nu \bar{S}_{i j}+T_{i j}\right),
$$

where

$$
T_{i j}=\bar{u}_{i} \bar{u}_{j}-\overline{u_{i} u_{j}}
$$

is the subgrid-stresses tensor, responsible of momentum exchanges between the subgrid- and the filtered scales. The filtered continuity equation writes

$$
\frac{\partial \bar{u}_{j}}{\partial x_{j}}=0
$$

Let us consider now the mixing of a passive scalar (such as temperature or density) of molecular diffusivity $\kappa$ transported by the flow, and satisfying the equation

$$
\frac{\partial \rho}{\partial t}+\frac{\partial}{\partial x_{j}}\left(\rho u_{j}\right)=\frac{\partial}{\partial x_{j}}\left\{\kappa \frac{\partial \rho}{\partial x_{j}}\right\}
$$

The filtered scalar equation writes

$$
\frac{\partial \bar{\rho}}{\partial t}+\frac{\partial}{\partial x_{j}}\left(\bar{\rho} \bar{u}_{j}\right)=\frac{\partial}{\partial x_{j}}\left\{\kappa \frac{\partial \bar{\rho}}{\partial x_{j}}+T_{j}^{(\rho)}\right\},
$$

where

$$
T_{j}^{(\rho)}=\bar{\rho} \bar{u}_{j}-\overline{\rho u_{j}}
$$

is the subgrid scalar flux. $T_{i j}$ and $T_{j}^{(\rho)}$ can be written as:

$$
\begin{aligned}
T_{i j} & =-\left(\overline{u_{i}^{\prime} u_{j}^{\prime}}+\overline{\bar{u}_{i} u_{j}^{\prime}}+\overline{u_{i}^{\prime} \bar{u}_{j}}+\overline{\bar{u}_{i} \bar{u}_{j}}-\bar{u}_{i} \bar{u}_{j}\right), \\
T_{j}^{(\rho)} & =-\left(\overline{\rho^{\prime} u_{j}^{\prime}}+\overline{\bar{\rho} u_{j}^{\prime}}+\overline{\rho^{\prime} \bar{u}_{j}}+\bar{\rho} \overline{\bar{u}_{j}}-\bar{\rho} \bar{u}_{j}\right) .
\end{aligned}
$$

In equation $(3.10),-\overline{u_{i}^{\prime} u_{j}^{\prime}}$ is a Reynolds-stress like term, $-\left(\overline{\bar{u}_{i} u_{j}^{\prime}}+\overline{u_{i}^{\prime} \bar{u}_{j}}\right)$ is called the Clark term (Clark et al. [21]), and $\bar{u}_{i} \bar{u}_{j}-\overline{\bar{u}}_{i} \bar{u}_{j}$ is the Leonard's 
tensor (Leonard [65]). The latter is explicit in the sense that it is defined in terms of the filtered field, and has been used in scale-similarity models to provide information on the subgrid stresses (see Sect. 7.3). Leonard's stresses are also a major ingredient of the so-called Germano's identity for the dynamic approach in physical space (see Sect. 6).

These subgridscale tensors and fluxes need of course to be modelled.

\subsection{LES Boussinesq equations in a rotating frame}

We give now the LES equations corresponding to Navier-Stokes equations within the Boussinesq approximation in a Cartesian frame of reference rotating with a constant angular velocity $\Omega$ about the $x_{3}$ axis:

$$
\begin{aligned}
& \frac{\partial \bar{u}_{i}}{\partial t}+\frac{\partial}{\partial x_{j}}\left(\bar{u}_{i} \bar{u}_{j}\right)=-\frac{1}{\rho_{0}} \frac{\partial \bar{p}}{\partial x_{i}}+\frac{\partial}{\partial x_{j}}\left(2 \nu \bar{S}_{i j}+T_{i j}\right) \\
& +2 \epsilon_{i j 3} \Omega \bar{u}_{j}-g_{i} \delta_{i 3} \frac{\bar{\rho}}{\rho_{0}} \\
& \frac{\partial \bar{\rho}}{\partial t}+\frac{\partial}{\partial x_{j}}\left(\bar{\rho} \bar{u}_{j}\right)=\frac{\partial}{\partial x_{j}}\left\{\kappa \frac{\partial \bar{\rho}}{\partial x_{j}}+T_{j}^{(\rho)}\right\} .
\end{aligned}
$$

This comes from the filtering of a particular version of Boussinesq equations, valid both for a liquid and a perfect gas, where $\rho$ is the total density, $p$ the static pressure, and $\rho_{0}$ an average density on the height of the fluid layer. $T_{i j}$ and $T_{j}^{(\rho)}$ are defined as above. Here, the density is still a scalar transported by the flow (as in Eq. (3.7)). But it is not passive since it reacts through gravity in the momentum equation. In fact, this system of equations is very useful to study stably-stratified or thermally convective rotating flows.

\subsection{Eddy-viscosity and diffusivity assumption}

By analogy with what is done in the framework of Reynolds equations for the ensemble-averaged equations, the subgridscale tensors are in most of the cases expressed in terms of eddy-viscosity and diffusivity coefficients in the form

$$
T_{i j}=2 \nu_{\mathrm{t}}(\vec{x}, t) \bar{S}_{i j}+\frac{1}{3} T_{l l} \delta_{i j} ; \quad T_{j}^{(\rho)}=\kappa_{\mathrm{t}}(\vec{x}, t) \frac{\partial \bar{\rho}}{\partial x_{j}} .
$$

Then LES equations for a flow of uniform density write

$$
\begin{aligned}
\frac{\partial \bar{u}_{i}}{\partial t}+\frac{\partial}{\partial x_{j}}\left(\bar{u}_{i} \bar{u}_{j}\right) & =-\frac{1}{\rho_{0}} \frac{\partial \bar{P}}{\partial x_{i}}+\frac{\partial}{\partial x_{j}}\left\{\left(\nu+\nu_{\mathrm{t}}\right)\left(\frac{\partial \bar{u}_{i}}{\partial x_{j}}+\frac{\partial \bar{u}_{j}}{\partial x_{i}}\right)\right\} \\
\frac{\partial \bar{\rho}}{\partial t}+\frac{\partial}{\partial x_{j}}\left(\bar{\rho} \bar{u}_{j}\right) & =\frac{\partial}{\partial x_{j}}\left\{\left(\kappa+\kappa_{\mathrm{t}}\right) \frac{\partial \bar{\rho}}{\partial x_{j}}\right\}
\end{aligned}
$$


where

$$
\bar{P}=\bar{p}-\frac{1}{3} \rho_{0} T_{l l}
$$

is a modified pressure which can be determined with the aid of the filtered continuity equation.

Two questions are in fact posed: the first one is how to determine the eddy-viscosity $\nu_{\mathrm{t}}$ and the corresponding turbulent Prandtl number

$$
P r_{\mathrm{t}}=\frac{\nu_{\mathrm{t}}}{\kappa_{\mathrm{t}}}
$$

and the second one concerns the validity of the eddy-viscosity assumption itself. Indeed, it is based on an analogy with Newtonian fluids, which is certainly not fulfilled here. Let us discuss briefly this point: molecular viscosity $\nu$ characterizes for a "macroscopic" fluid parcel the momentum exchanges with the surrounding fluid due to molecular diffusion across its interface. Here, one assumes a wide separation between macroscopic and microscopic scales and it is this separation which allows to calculate these molecular exchange coefficients using kinetic theories of liquids or gases where molecules are assumed to follow some sort of Gaussian random walk. No such scale separation exists in the LES problem, where one observes in general a distribution of energy (kinetic-energy spectrum) continuously decreasing from the energetic to the smallest dissipative scales, even in inflexional shear flows with vigorous coherent vortices. Since the cutoff scale $\Delta x$ is in the middle of this spectrum, there is obviously no spectral gap at this level. On the other hand, trajectories of fluid parcels are very far from a random walk, since they may be either trapped around a vortex or strained in stagnation regions between vortices.

We believe therefore that the lack of spectral gap is the major drawback of the eddy-viscosity assumption, responsible for the fact that numerous numerical and even experimental a priori tests (see e.g. Clark et al. [21], Liu et al. [77]) invalidate relation (3.14): when a low-pass filter is for instance applied to DNS results, one can calculate explicitely the subgrid-stresses tensors, and correlate them to the filtered deformation. The correlation found is very poor, of the order of 0.1 instead of 1 . This justifies the development of models going beyond the classical eddy-viscosity concept: it is the case of the spectral eddy viscosity (see Sect. 4.1), and also of models presented in Section 5. However, LES results based on classical eddy-viscosities in physical space derived from Smagorinsky or structure-function models may give very good results, as will be seen below, from the point of view of vortex dynamics and statistical predictions. 


\subsection{Smagorinsky's model}

As already pointed out, the most widely used eddy-viscosity model was proposed by Smagorinsky [105]. He introduced an eddy-viscosity which was supposed to model subgridscale dissipation through a Kolmogorov $k^{-5 / 3}$ cascade. Smagorinsky's model is an adaptation of Prandtl's mixing-length theory to subgridscale modelling. Prandtl assumes that the eddy viscosity arising in Reynolds-averaged Navier-Stokes equations is proportional to a scale characteristic of turbulence (the mixing length) times a characteristic turbulent velocity. In the same way, Smagorinsky assumes that the LES eddy-viscosity is proportional to the subgrid-scale characteristic length scale $\Delta x$, and to a characteristic subgrid-scale velocity

$$
v_{\Delta x}=\Delta x|\bar{S}|,
$$

based on the second invariant of the filtered-field deformation tensor

$$
|\bar{S}|=\sqrt{2 \bar{S}_{i j} \bar{S}_{i j}} .
$$

Thus Smagorinsky's eddy viscosity writes

$$
\nu_{\mathrm{t}}=\left(C_{\mathrm{S}} \Delta x\right)^{2}|\bar{S}| .
$$

If one assumes that $k_{\mathrm{C}}=\pi / \Delta x$, the cutoff wavenumber in Fourier space, lies within a $k^{-5 / 3}$ Kolmogorov cascade $E(k)=C_{\mathrm{K}} \epsilon^{2 / 3} k^{-5 / 3}\left(C_{\mathrm{K}}\right.$ is the Kolmogorov constant), one can analytically determine the constant $C_{\mathrm{S}}$. It is then found (see Lilly [75]):

$$
C_{\mathrm{S}} \approx \frac{1}{\pi}\left(\frac{3 C_{\mathrm{K}}}{2}\right)^{-3 / 4} .
$$

It yields $C_{\mathrm{S}} \approx 0.18$ for a Kolmogorov constant of 1.4 . This value proves to give acceptable results for LES of isotropic turbulence. However, most researchers prefer $C_{\mathrm{S}}=0.1$ (which represents a reduction by nearly a factor of 4 of the eddy-viscosity), a value for which Smagorinsky's model behaves reasonably well for free-shear flows and for channel flow ${ }^{1}$ (Moin and Kim [85]). This clearly indicates that $C_{\mathrm{S}}$ is not a universal constant, and that assuming $k_{\mathrm{C}}$ within a $k^{-5 / 3}$ Kolmogorov cascade is too much a constraint. In fact, Smagorinsky's model is obviously too dissipative in the presence of a wall, and does not work in particular for transition in a boundary-layer developing upon a flat plate: it artificially relaminarizes the flow if the upstream perturbation is not high enough. This is due to

\footnotetext{
${ }^{1}$ These channel flow computations require wall laws.
} 
the heavy influence in the eddy-viscosity expression of the velocity gradient in the direction normal to the wall (see Meneveau and Katz [82]). Furthermore, the subgrid-scale dissipation $P_{\mathrm{sm}}=T_{i j} \bar{S}_{i j}$ is on the average positive corresponding to a global flow of kinetic energy from the large-scale towards the subgrid scale (forward scatter). However, backscatter, associated with a locally negative $P_{\mathrm{sm}}$ and with a reversed energy transfer, occurs in some flow regions (Piomelli et al. [95], Liu et al. [77]). Classical eddy-viscosity models like Smagorinsky's assume that $P_{\mathrm{sm}}$ is positive everywhere. This justifies Smagorinsky's dynamic approach which will be presented in Section 6 , and where the constant is dynamically adjusted to the flow conditions.

\section{LES in Fourier space}

\subsection{Spectral eddy viscosity and diffusivity}

We assume that Navier-Stokes is written in Fourier space. This requires statistical homogeneity in the three directions of space, but we will see below how to handle flows with only one direction of inhomogeneity. Let $\hat{u}_{i}(\vec{k}, t)$ and $\hat{\rho}(\vec{k}, t)$ be the spatial Fourier transform of the velocity and passive-scalar fields where $\vec{k}$ is the wavenumber. The filter consists in a sharp cut-off filter, simply clipping all the modes larger than $k_{\mathrm{C}}$, where $k_{\mathrm{C}}=\pi / \Delta x$ is still the cut-off wavenumber.

We write Navier-Stokes in Fourier space as

$$
\begin{aligned}
\frac{\partial}{\partial t} \hat{u}_{i}(\vec{k}, t) & +\left[\nu+\nu_{\mathrm{t}}\left(\vec{k} \mid k_{\mathrm{C}}\right)\right] k^{2} \hat{u}_{i}(\vec{k}, t) \\
= & -i k_{m} P_{i j}(\vec{k}) \int_{|\vec{p}|,|\vec{q}|<k_{\mathrm{C}}}^{\vec{p}+\vec{q}=\vec{k}} \hat{u}_{j}(\vec{p}, t) \hat{u}_{m}(\vec{q}, t) \mathrm{d} \vec{p}
\end{aligned}
$$

where $P_{i j}(\vec{k})=\delta_{i j}-\left(k_{i} k_{j} / k^{2}\right)$ is the projector on the plane perpendicular to $\vec{k}$, which allows in particular to eliminate the pressure. The spectral eddy viscosity $\nu_{\mathrm{t}}\left(\vec{k} \mid k_{\mathrm{C}}\right)$ is defined by

$$
\nu_{\mathrm{t}}\left(\vec{k} \mid k_{\mathrm{C}}\right) k^{2} \hat{u}_{i}(\vec{k}, t)=i k_{m} P_{i j}(\vec{k}) \int_{|\vec{p}|_{\text {or }|\vec{q}|>k_{\mathrm{C}}}}^{\vec{p}+\vec{q}=\vec{k}} \hat{u}_{j}(\vec{p}, t) \hat{u}_{m}(\vec{q}, t) \mathrm{d} \vec{p} .
$$

The r.h.s. of equation (4.1) corresponds to a resolved transfer.

A spectral eddy-diffusivity for the passive scalar may be defined in the same way, by writing the passive-scalar equation in Fourier space

$$
\frac{\partial}{\partial t} \hat{\rho}(\vec{k}, t)+\left[\kappa+\kappa_{\mathrm{t}}\left(\vec{k} \mid k_{\mathrm{C}}\right)\right] k^{2} \hat{\rho}(\vec{k}, t)=-i k_{j} \int_{|\vec{p}|,|\vec{q}|<k_{\mathrm{C}}}^{\vec{p}+\vec{q}=\vec{k}} \hat{u}_{j}(\vec{p}, t) \hat{\rho}(\vec{q}, t) \mathrm{d} \vec{p}
$$


with

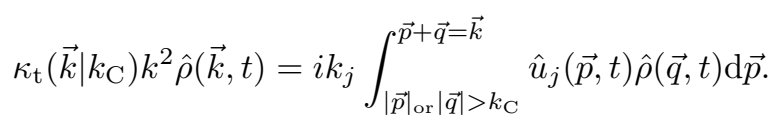

\subsection{EDQNM plateau-peak model}

Expressions (4.2) and (4.4) give exact expressions of the eddy coefficients. They are however useless since they involve subgrid quantities. In fact, they can be evaluated at the level of kinetic-energy and passive-scalar spectra evolution equations obtained with the aid of two-point closures of threedimensional isotropic turbulence. It is in this context that the concept of $k$-dependent eddy-viscosity was first introduced by Kraichnan [54]. The spectral eddy-diffusivity for a passive scalar was introduced by Chollet and Lesieur $[19,20]$.

Kraichnan used the so-called Test-Field Model. We work using a slightly different closure called the Eddy-Damped Quasi-Normal Markovian (EDQNM) theory introduced by Orszag [89,90] (see also André and Lesieur [3], and Lesieur [67], for details). In this theory, which is easily handable only in the case of isotropic turbulence, the fourth-order cumulants in the hierarchy of moments equations are supposed to relax linearly the thirdorder moments, in the same qualitative way as does the molecular viscosity. The EDQNM provides for isotropic turbulence a closed equation of evolution for the kinetic energy spectrum $E(k, t)$. In a LES approach, we split the transfers between interactions involving only modes smaller than $k_{\mathrm{C}}$, and the others. The equations for the supergrid-scale velocity, $\bar{E}(k, t)$, and scalar, $\bar{E}_{\rho}(k, t)$ spectra then write

$$
\begin{aligned}
\left(\frac{\partial}{\partial t}+2 \nu k^{2}\right) \bar{E}(k, t) & =T_{<k_{\mathrm{C}}}(k, t)+T_{>k_{\mathrm{C}}}(k, t) \\
\left(\frac{\partial}{\partial t}+2 \kappa k^{2}\right) \bar{E}_{\rho}(k, t) & =T_{<k_{\mathrm{C}}}^{\rho}(k, t)+T_{>k_{\mathrm{C}}}^{\rho}(k, t),
\end{aligned}
$$

where $T_{<k_{\mathrm{C}}}(k, t)$ and $T_{<k_{\mathrm{C}}}^{\rho}(k, t)$ are the spectral transfers corresponding to resolved triads such that $k, p, q \leq k_{\mathrm{C}}$, and $T_{>k_{\mathrm{C}}}\left(\right.$ resp. $T_{>k_{\mathrm{C}}}^{\rho}$ ) to modes such that $k<k_{\mathrm{C}}, p$ and (or) $q>k_{\mathrm{C}}$.

We first assume that $k_{\mathrm{C}}$ lies within a $k^{-5 / 3}$ inertial range. For $k \ll k_{\mathrm{C}}$, both modes being larger than $k_{i}$ the kinetic-energy peak, expansions in powers of the small parameter $k / k_{\mathrm{C}}$ yield to the lowest order

$$
T_{>k_{\mathrm{C}}}(k, t)=-2 \nu_{\mathrm{t}}^{\infty} k^{2} \bar{E}(k, t)
$$




$$
T_{>k_{\mathrm{C}}}^{\rho}(k, t)=-2 \kappa_{\mathrm{t}}^{\infty} k^{2} \bar{E}_{\rho}(k, t)
$$

with

$$
\begin{gathered}
\nu_{\mathrm{t}}^{\infty}=0.441 C_{\mathrm{K}}^{-3 / 2}\left[\frac{E\left(k_{\mathrm{C}}\right)}{k_{\mathrm{C}}}\right]^{1 / 2} \\
\kappa_{\mathrm{t}}^{\infty}=\frac{\nu_{\mathrm{t}}^{\infty}}{P_{\mathrm{r}}^{(t)}} ; \quad P_{\mathrm{r}}^{(t)}=0.6 .
\end{gathered}
$$

Here, $E\left(k_{\mathrm{C}}\right)$ is the kinetic-energy spectrum at the cutoff $k_{\mathrm{C}}$. The 0.6 value for the Prandtl number is in fact the highest one permitted by the choice of two further adjustable constants arising in the EDQNM passive-scalar equation (see Lesieur [67]). When $k$ is close to $k_{\mathrm{C}}$, the numerical evaluation of the EDQNM transfers yields

$$
\begin{aligned}
& T_{>k_{\mathrm{C}}}(k, t)=-2 \nu_{\mathrm{t}}\left(k \mid k_{\mathrm{C}}\right) k^{2} \bar{E}(k, t) \\
& T_{>k_{\mathrm{C}}}^{\rho}(k, t)=-2 \kappa_{\mathrm{t}}\left(k \mid k_{\mathrm{C}}\right) k^{2} \bar{E}_{\rho}(k, t),
\end{aligned}
$$

with

$$
\nu_{\mathrm{t}}\left(k \mid k_{\mathrm{C}}\right)=K\left(\frac{k}{k_{\mathrm{C}}}\right) \nu_{\mathrm{t}}^{\infty} ; \quad \kappa_{\mathrm{t}}\left(k \mid k_{\mathrm{C}}\right)=C\left(\frac{k}{k_{\mathrm{C}}}\right) \kappa_{\mathrm{t}}^{\infty}
$$

where $\nu_{\mathrm{t}}^{\infty}$ and $\kappa_{\mathrm{t}}^{\infty}$ are the asymptotic values given by equations (4.9) and (4.10), and $K(x)$ and $C(x)$ nondimensional functions equal to 1 for $x=0$. As shown also by Kraichnan [54], $K(x)$ has a plateauvalue at 1 up to $k / k_{\mathrm{C}} \approx 1 / 3$. Above, it displays a strong peak (cusp-behaviour). Let us mention that Kraichnan did not point out the scaling of the eddy viscosity against $\left[E\left(k_{\mathrm{C}}\right) / k_{\mathrm{C}}\right]^{1 / 2}$, which turns out to be essential for LES purposes. Indeed, when the energy spectrum decreases rapidly at infinity (for instance during the initial stage of decay in isotropic turbulence, see below), the eddy viscosity will be very low and inactive. On the other hand, we have $\left[E\left(k_{\mathrm{C}}\right) / k_{\mathrm{C}}\right]^{1 / 2}$ $\sim \epsilon^{1 / 3} k_{\mathrm{C}}^{-4 / 3}$ in an inertial-range expression, which may be important even before the establishment of the $k^{-5 / 3}$ range. Furthermore, we will show below that the plateau-peak model may be generalized to spectra different from Kolmogorov at the cutoff (spectral-dynamic model).

It was shown by Chollet and Lesieur $[19,20]$ that $C(x)$ behaves qualitatively as $K(x)$ (plateau at 1 and positive peak), and that the spectral turbulent Prandtl number $\nu_{\mathrm{t}}\left(k \mid k_{\mathrm{C}}\right) / \kappa_{t}\left(k \mid k_{\mathrm{C}}\right)$ is approximately constant, and thus equal to 0.6 as given by equation (4.10).

It is clear that the plateau part corresponds to the usual eddy-coefficients assumption when one goes back to physical space, so that the "peak" part 
goes beyond the scale-separation assumption inherent to the classical eddyviscosity and diffusivity concepts. The peak is mostly due to semi-local interactions across $k_{\mathrm{C}}$ : near the cutoff wavenumber, the main nonlinear interactions between the resolved and unresolved scales involve the smallest eddies of the former and the largest eddies of the latter (such that $p \ll k \sim$ $\left.q \sim k_{\mathrm{C}}\right)$.

At the level of kinetic-energy exchanges, this formulation of the spectral eddy-viscosity includes all backscatter effects in the following sense: when kinetic energy is injected around a particular wavenumber $k_{i}$, and for the decaying case, it may be shown with the aid of expansions in terms of the small parameter $k / k_{i} \ll 1$ that the transfer is proportional to $k^{4}$, and hence a spectrum proportional to $k^{4}$ is produced at low wavenumbers $k \ll k_{i}$ (see Lesieur and Schertzer [72]). Such a backscatter transfer is due to nonlinear resonance between two energetic modes in the neighborhood of $k_{i}$. This was checked in an LES in which $k_{i}$ was close to $k_{\mathrm{C}}$ by Lesieur and Rogallo [71]. Considering the eddy-viscosity (4.13), it may be shown that, for $k \ll k_{\mathrm{C}}$ (both modes being larger than $k_{i}$ and in the inertial range), the backscatter due to subgrid-scale modes is negligible. Indeed, its relative importance in terms of transfers is, according to EDQNM theory, $\left(k / k_{\mathrm{C}}\right)^{2}\left[E\left(k_{\mathrm{C}}\right) / E(k)\right]$, which is very small since $E\left(k_{\mathrm{C}}\right) \ll E(k)$ (see Lesieur [67]). The cusp results from the difference between a "drain", which sends energy to the subgrid scales, and a "backscatter" which injects energy back to the supergrid scales, so that the net effect is a positive eddy-viscosity.

As shown by Chollet [18], the plateau-peak behaviour of $K(x)$ can approximately be expresssed with the following analytical expression:

$$
K(x)=1+34.5 \mathrm{e}^{-3.03 / x} .
$$

We will see below another analytic expression of this spectral eddy viscosity in terms of hyper-viscosities (see Sect. 7.1).

The plateau-peak model consists in using these eddy-viscosities in the deterministic equations $((4.1),(4.3))$. One advantage of using such a subgridscale modelling is that they are correct from an energetic-transfer viewpoint.

\subsubsection{The spectral-dynamic model}

Another drawback of the plateau-peak model is that it is restricted to the case where $k_{\mathrm{C}}$ lies within a $k^{-5 / 3}$ Kolmogorov cascade. Fortunately, this can be cured with the introduction of the spectral-dynamic model. One assumes now that the kinetic-energy spectrum is $\propto k^{-m}$ for $k>k_{\mathrm{C}}$, whith $m$ not necessarily equal to $5 / 3$. We modify the spectral eddy viscosity as

$$
\nu_{\mathrm{t}}\left(k \mid k_{\mathrm{C}}\right)=0.31 C_{\mathrm{K}}^{-3 / 2} \sqrt{3-m} \frac{5-m}{m+1} K\left(\frac{k}{k_{\mathrm{C}}}\right)\left[\frac{E\left(k_{\mathrm{C}}\right)}{k_{\mathrm{C}}}\right]^{1 / 2},
$$


for $m \leq 3$. This expression is exact for $k \ll k_{\mathrm{C}}$ within the same nonlocal expansions of the EDQNM theory, as shown in Métais and Lesieur [80]. We retain the peak shape through $K\left(k / k_{\mathrm{C}}\right)$ in order to be consistent with the Kolmogorov spectrum expression of the eddy viscosity. For $m>3$, the scaling is no more valid, and the eddy viscosity will be set equal to zero. Indeed, we are very close to a DNS for such spectra. In the spectraldynamic model, the exponent $m$ is determined through the LES with the aid of least-squares fits of the kinetic-energy spectrum close to the cutoff. On may also check that the turbulent Prandtl number is given by:

$$
P_{\mathrm{r}}^{\mathrm{t}}=0.18(5-m)
$$

(see Métais and Lesieur [80]). This value does not depend on the Kolmogorov and model constants. It is a great advantage in LES of heated or variable-density flows to have the possibility of a variable turbulent Prandtl number. This possibility exists also for the dynamic models in physical space which will be presented in the following sections.

\subsubsection{Existence of the plateau-peak}

The spectral LES of decaying isotropic turbulence and associated scalar mixing performed by Lesieur and Rogallo [71], together with those of Métais and Lesieur [80], have been used to compute directly the spectral eddyviscosity and diffusivity. The method is the same as that employed by Domaradzki et al. [29] for a direct numerical simulation: one defines a fictitious cutoff wavenumber $k_{\mathrm{C}}^{\prime}=k_{\mathrm{C}} / 2$, across which the kinetic-energy transfer $T$ and scalar transfer $T^{\rho}$ are evaluated. Since we deal with a largeeddy simulation, the latter correspond to triadic interactions such that $k<$ $k_{\mathrm{C}}^{\prime}, p$ and (or) $q>k_{\mathrm{C}}^{\prime}$ and $p, q<k_{\mathrm{C}}$ : they are termed $T_{>k_{\mathrm{C}}^{\prime}}^{<k_{\mathrm{C}}}(k, t)$ and $T_{>k_{\mathrm{C}}^{\prime}}^{\rho<k_{\mathrm{C}}}(k, t)$. They correspond to resolved transfers, and satisfy energetic equalities of the type

$$
T_{>k_{\mathrm{C}}^{\prime}}^{<k_{\mathrm{C}}}(k, t)=T_{>k_{\mathrm{C}}^{\prime}}(k, t)-T_{>k_{\mathrm{C}}}(k, t)
$$

where $T_{>k_{\mathrm{C}}^{\prime}}$ and $T_{>k_{\mathrm{C}}}$ are the total kinetic energy transfers across $k_{\mathrm{C}}^{\prime}$ and $k_{\mathrm{C}}$. It is important to note that equation (4.17) is the exact energetic equivalent in spectral space of Germano's identity (Germano [41], see Sect. 6). A similar relation holds for $T_{k_{\mathrm{C}}^{\prime}}^{\rho<k_{\mathrm{C}}}$. Once divided by $-2 k^{2} E(k, t)$ and $-2 k^{2} E_{\rho}(k, t)$, they give the resolved spectral eddy-viscosity and diffusivity. Figure 7 shows these functions normalized by $\left[E\left(k_{\mathrm{C}}^{\prime}\right) / k_{\mathrm{C}}^{\prime}\right]^{1 / 2}$, taken from Métais and Lesieur [80]. Similar results had been found in Lesieur and Rogallo [71]. It demonstrates that the plateau-peak behaviour does exist for the eddy viscosity, but is questionable for the eddy diffusivity. This 
anomalous scalar range still exists in a DNS of decaying isotropic turbulence: in this case, the double filtering yields a plateau-peak eddy viscosity with a plateau value approximately 0, as was discovered by Domaradzki et al. [29]. The eddy-diffusivity on the contrary still behaves as in the LES. In fact, Métais and Lesieur [80] have checked that the anomaly disappears when the temperature is no more passive and coupled with the velocity within the frame of Boussinesq approximation (stable stratification). It is possible that the same holds for compressible turbulence, which would legitimate the use of the plateau-peak eddy diffusivity in this case. Note that the plateaupeak behaviour was recently confirmed by the experimental data from the turbulent wake experiment performed by Cerutti et al. [17]

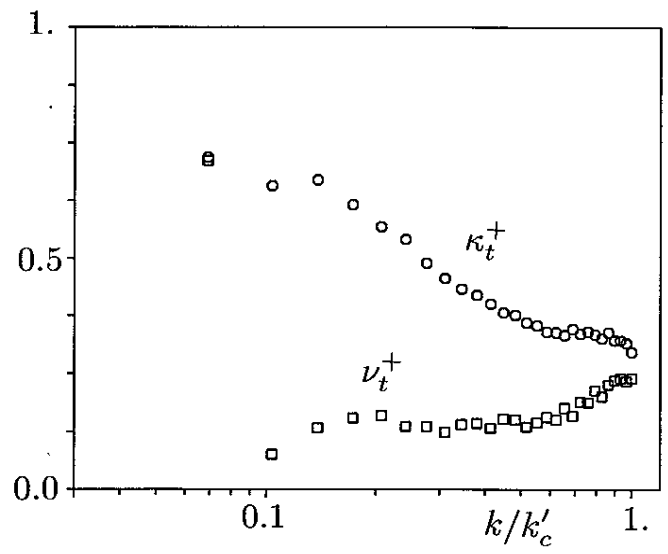

Fig. 7. Resolved eddy-viscosity and diffusivity evaluated through a double filtering in LES of isotropic decaying turbulence (from Métais and Lesieur [80]).

The plateau-peak spectral eddy-viscosity and diffusivity coefficients have been used successfully to study freely-decaying three-dimensional turbulence at high Reynolds number. LES at a resolution of $128^{3}$ Fourier modes have been carried out by Lesieur and Rogallo [71]. It was checked that, at first, Kolmogorov and Corrsin-Oboukhov $k^{-5 / 3}$ cascades establish. Afterwards, the kinetic-energy spectrum decays self-similarly, with a spectral slope comprised between $-5 / 3$ and -2 . The scalar spectrum exhibits a very short inertial-convective range close to the cutoff, and a very wide range shallower than $k^{-1}$ in the large scales. Here, the scalar decays much faster than the temperature. This anomalous range was explained by Métais and Lesieur [80] as due to the quasi two-dimensional character of the scalar diffusion in the large scales, leading to large-scale intermittency of the scalar. More precisely, the scalar diffusion seems to be dominated by the effect of 
coherent vortices already considered in Section 2.1, and tending to form scalar discontinuities in the stagnation regions between the vortex tubes.

The plateau-peak model been applied to various temporally growing freeshear flows like wakes, jets and mixing-layers for which the flow is assumed to be periodic in the streamwise direction. Silvestrini ([104], see also Lesieur and Métais [69]) showed that LES, even at moderate resolution are able to reproduce the complexity of the mixing-layer dynamics. With threedimensional initial noise, the helical pairing arrangement, previously found in the mixing-layer DNS of Comte et al. [24], was recovered: vortex filaments oscillate out-of-phase in the spanwise direction, and reconnect, yielding a vortex-lattice structure. On the other hand, and if the perturbation is quasi two-dimensional, the mixing layer was shown to evolve into a set of big quasi two-dimensional Kelvin-Helmholtz vortices which both undergo pairing and stretch intense longitudinal hairpin vortices in the stagnation regions between them. This stretching of longitudinal vortices had been observed experimentally for a long time (see e.g. Bernal and Roshko [11]). It is remarkable that LES, done at a quite low resolution, are indeed able to capture the longitudinal vortices, which are at quite small scales. The DNS of Comte et al. [24] at an equivalent resolution were unable to find organized intense longitudinal vortices, because their molecular Reynolds number is too low. It is only at a much higher resolution that DNS can capture these vortices (Rogers and Moser [97]). This is an example where large-eddy simulations are an excellent tool to capture not only large- but also small-scale vortices.

However, a spectral eddy-viscosity is difficult to employ when the geometry of the problem obliges one to work in physical space. This has motivated the development of new models directly inspired from the previous spectral formulation but which can be utilized in physical space (see Sect. 5.1).

\subsection{Incompressible plane channel}

We show now how the spectral dynamic model may be applied to an incompressible turbulent Poiseuille flow between two infinite parallel flat plates. A schematic view of the channel is presented in Figure 8. A rotation axis oriented in the spanwise direction is indicated for further applications, but rotation is inactive right now. The channel has a width $2 h$, and we define the macroscopic Reynolds number by $R e=2 h U_{\mathrm{m}} / \nu$, where $U_{\mathrm{m}}$ is the bulk velocity. We assume periodicity in the streamwise and spanwise directions. Calculations are carried out at constant $U_{\mathrm{m}}$. They are initiated by a parabolic laminar profile perturbed by a small three-dimensional random noise, and pursued up to complete statistical stationarity. 

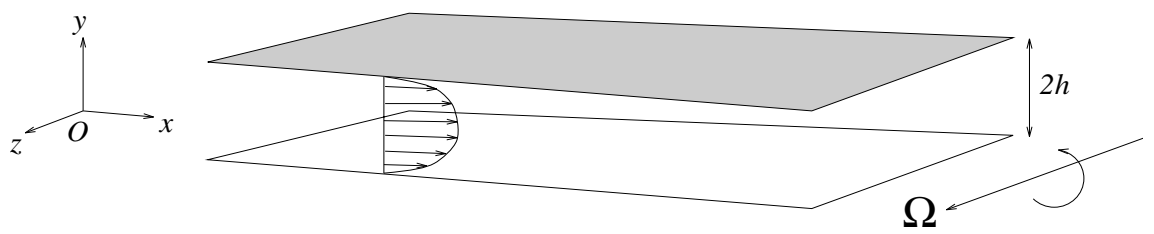

Fig. 8. Schematic view of a plane channel.

\subsubsection{Wall units}

Let us recall the so-called wall units, very useful when turbulence has developed. The friction velocity $v_{*}$ is defined by

$$
v_{*}^{2}=\left.\nu \frac{\mathrm{d}\langle u\rangle}{\mathrm{d} y}\right|_{y=0},
$$

with the aid of the mean-velocity derivative at the wall. The velocities will be normalized by $v_{*}$ and noted $u_{i}^{+}=u_{i} / v_{*}$. One defines also a viscous thickness

$$
l_{\mathrm{v}}=\frac{\nu}{v_{*}},
$$

characteristic of motions very close to the wall which are dominated by viscosity, so that the spatial scales will be normalized by $l_{\mathrm{v}}$ and noted $x_{i}^{+}=$ $x_{i} / l_{\mathrm{v}}$. Let $y$ be the distance perpendicular to the wall. Substituting Taylorseries expansions of the velocity components in powers of $y^{+}$close to the wall, together with the use of the continuity equation, leads to the result that $u^{+}$and $w^{+}$scale like $y^{+}$, while $v^{+}$scales likes $y^{+^{2}}$.

A consequence is that the mean longitudinal velocity profile scales also like $y^{+}$, behaviour which persists up to about $y^{+}=4 \approx 5$, which characterizes the width of the viscous region. DNS and LES show that this region is certainly not laminar, and strongly marked by the system of high and low longitudinal velocity streaks.

\subsubsection{Streaks and hairpins}

These coherent structures have been discovered experimentally in a turbulent boundary layer by Kline et al. [52], and had been observed previously by Klebanoff et al. [51] in a celebrated paper related to transition in a boundary layer forced upstream by a vibrating ribbon. Klebanoff associated the streaks (which he could detect with anemometers) to a system of longitudinal hairpins travelling downstream in phase and pumping between their 
legs fluid lower fluid slowed by the wall: this model explains the formation of low-speed streaks in the "peaks" of the hairpins, and high-speed streaks in the "valleys". The system of streaks in a turbulent channel was recovered numerically in the LES of Moin and Kim [85] already mentioned before and using Smagorinsky's model with wall laws.

\subsubsection{Spectral DNS and LES}

We will present turbulent channel DNS and LES, taken from the work of Lamballais [57] and Lamballais et al. [61] (see also [58-60]). It is interesting to see that $h^{+}=v_{*} h / \nu$ defines a microscopic Reynolds number based on the friction velocity. The numerical code used combines pseudo-spectral methods in the streamwise and spanwise directions, and compact finite-difference schemes of sixth order (see [64]) in the transverse direction with grid refinement close to the walls. The subgrid model is the spectral-dynamic eddy viscosity, computed thanks to two-dimensional kinetic-energy spectra calculated at each time step by spatial averages in planes parallel to the wall. Therefore, the exponent $m$ in the eddy viscosity depends of $y$ and $t$. This spectral eddy viscosity is implemented spectrally in the directions parallel to the wall, and in physical space in the transverse direction. This is a very precise code of accuracy comparable to a spectral method at equivalent resolution as shown by the comparison of DNS at $h^{+}=162$ with spectral DNS of Kuroda [56] at $h^{+}=150$. We reproduce this picture. We see in Figure $9 \mathrm{a}$ that the logarithmic range starts at $y^{+}=30$. Figure $9 \mathrm{~b}$ presents the rms velocity profiles as a function of $y^{+}$. It confirms the strong $u^{\prime}$ production close to the wall, with a peak at $y^{+}=12$, and which is obviously the signature of high- and low-speed streaks discussed before. Figures 9e (Reynolds stresses) and 9d (rms pressure fluctuations) have a peak higher $\left(y^{+} \approx 30\right)$. It might correspond to the tip of hairpin vortices ejected above the low-speed streaks. Figure $9 f$ corresponds to rms vorticity fluctuations. It indicates that the maximum vorticity produced is spanwise and at the wall $^{2}$. The vorticity perpendicular to the wall is about $40 \%$ higher than the longitudinal vorticity in the region $5<y^{+}<30$, which shows only a weak longitudinal vorticity stretching by the ambient shear.

We next present two LES using the spectral-dynamic model, at $R e=$ $6666\left(h^{+}=204\right.$, case A $)$ and $R e=14000\left(h^{+}=389\right.$, case B $)$. They are respectively subcritical and supercritical with respect to the linear-stability analysis of the Poiseuille profile. In the two simulations there is a grid refinement close to the wall, in order to simulate accurately the viscous

\footnotetext{
${ }^{2}$ It corresponds in fact to a steepening of $\mathrm{d} u / \mathrm{d} y$ at the wall under the high-speed streaks, resulting from a sort of squashing of the boundary layer upon the wall consecutive to the fact that the fluid is descending, and inducing an increase of the friction-coefficient.
} 

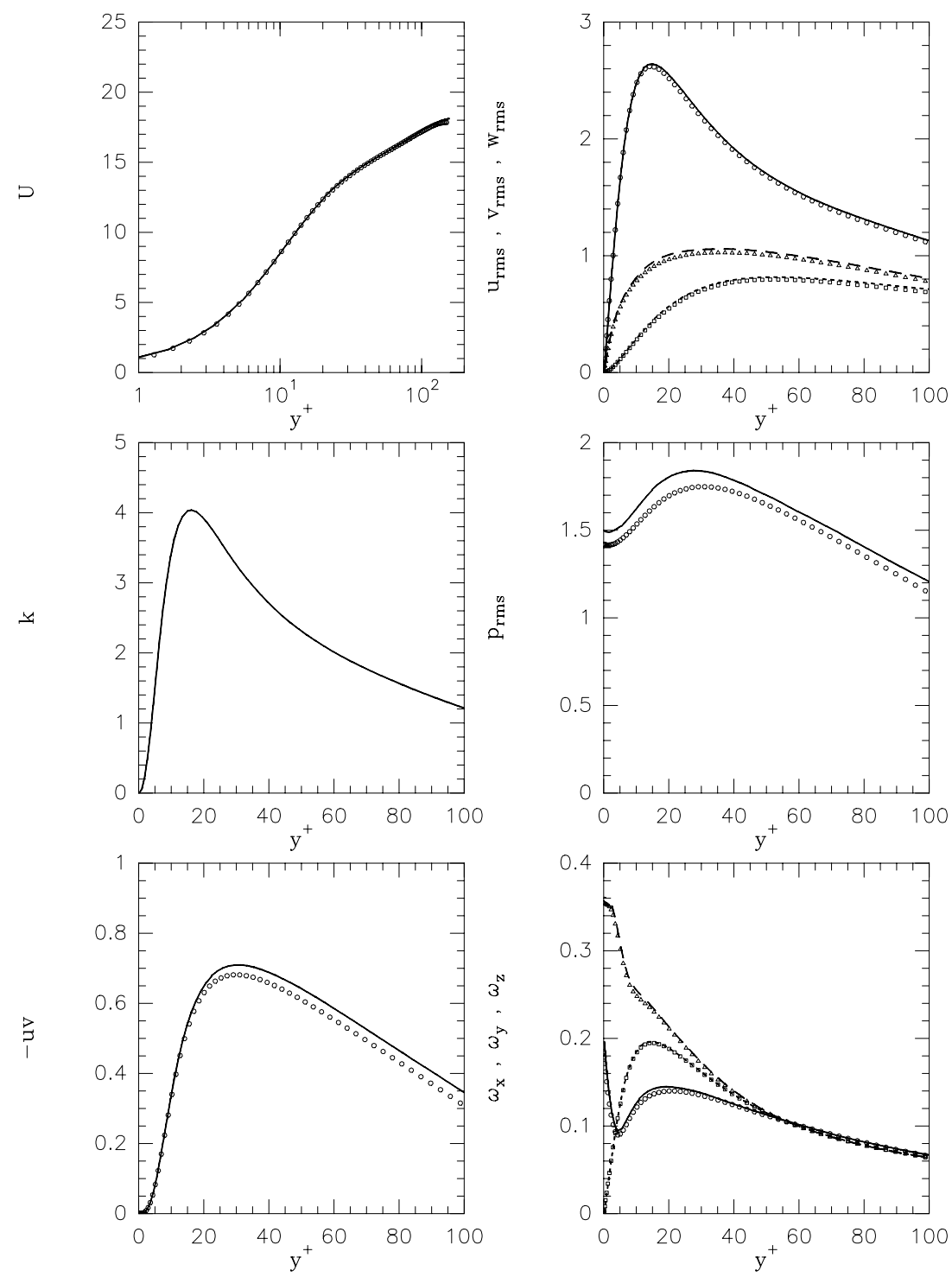

Fig. 9. Statistical data obtained in DNS of a turbulent channel flow by Lamballais (straight line) and Kuroda (symbols); from left to right and top to bottom, a) mean velocity, b) rms velocity fluctuations (respectively from top to bottom, longitudinal, spanwise, vertical), c) kinetic energy, d) rms pressure fluctuation, e) Reynolds stresses, f) rms vorticity (from top to bottom, spanwise, vertical, longitudinal). Courtesy Lamballais. 


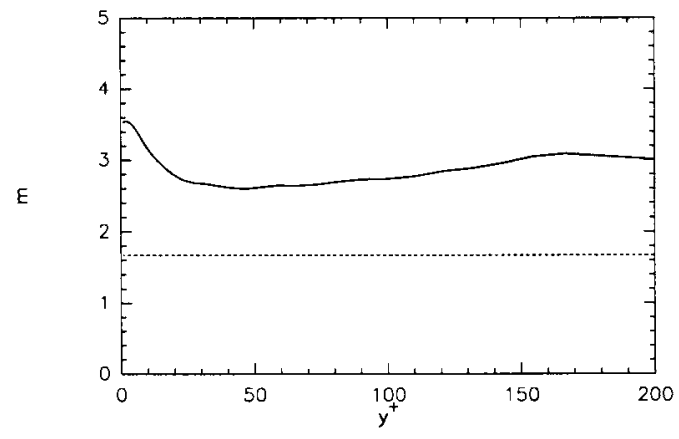

Fig. 10. Spectral-dynamic LES of the channel flow (case A), time-averaged exponent $m\left(y^{+}\right)$of the kinetic-energy spectrum at the cutoff.

sublayer. Figure 10 shows for case A the time-averaged exponent $m$ arising in the energy spectrum at the cutoff, as a function of the distance to the wall $y^{+}$. Regions where $m>3$ correspond to a zero eddy viscosity and hence a direct-numerical simulation. This is the case in particular close to the wall, up to $y^{+} \approx 12$ where we know that longitudinal velocity fluctuations are very intense, due to the low- and high-speed streaks. Therefore, and since the first point is very close to the wall $\left(y^{+}=1\right)$, such LES have the interesting property of becoming DNS in the vicinity of the wall, enabling to capture events which occur in this region.
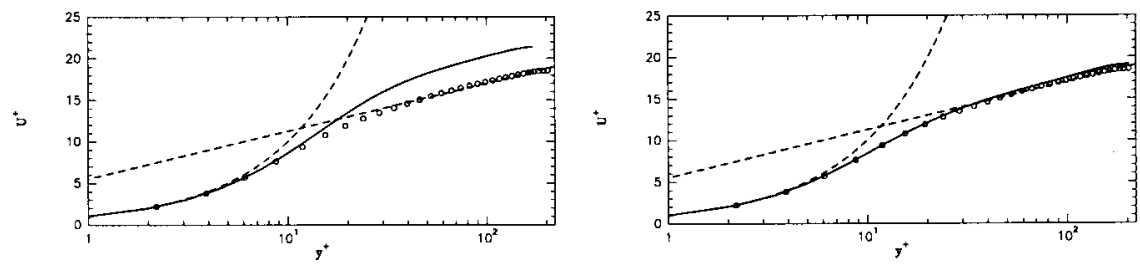

Fig. 11. Mean velocity profiles in wall units. Lines: spectral eddy-viscosity based simulations $(R e=6666)$; symbols: Piomelli $([94] ; R e=6500)$. Left: $m=5 / 3$ $\left(h^{+}=181\right)$; right: dynamic evaluation of $m(y, t)\left(h^{+}=204\right)$. The dashed straight line corresponds to the universal logarithmic mean velocity profile $\langle u\rangle=2.5 \ln y^{+}$ +5.5 .

Figure 11 shows (in semi-logarithmic coordinates) the mean velocity profile in case A, compared with the LES of Piomelli [94] using the dynamic 
model of Germano [41]. The latter is known to agree very well with experiments at these low Reynolds numbers. The simulation using the spectral dynamic model (right part of the figure) coincides with the DNS, yielding a correct value of the additive constant 5.5 in the logarithmic velocity profile. On the other hand, the LES using the classical spectral-cusp model with $m=5 / 3$ (left of figure) gives an error of $100 \%$ for this constant. The dashed parabola corresponds to the linear profile at the wall, which is exact up to 4 wall units. In case A, it was ckecked (see Lamballais et al. [61]) that the mean velocity and rms velocity fluctuations, compared very well with the dynamic-model predictions of Piomelli [94]. The agreement of rms velocities is still very good, with a correct prediction of the longitudinal velocity fluctuations peak.

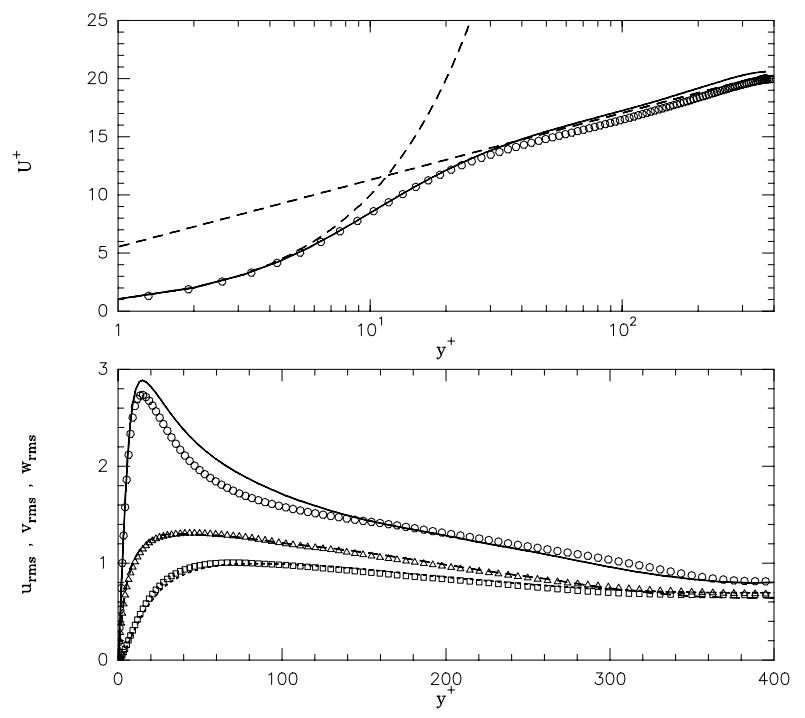

Fig. 12. Turbulent channel flow, comparisons of the spectral-dynamic model (solid lines, $h^{+}=389$ ) with the DNS of Antonia et al. ([4], symbols, $h^{+}=395$ ); top, mean velocity, bottom, rms velocity components.

Concerning the supercritical case, the LES of case B are in very good agreement with a DNS at $h^{+}=395$ carried out in Antonia et al. [4], both for the mean velocity and the rms velocity components. They are shown in Figure 12. Notice that the LES allows in this case to reduce the computational cost by a factor of the order of 100 , which is huge. Notice also that the extent of the linear-velocity profile range close to the wall has slightly increased (from 4 to 5) with the Reynolds number. We present finally in Figure 13 a map of the vorticity modulus at the same threshold for cases A 

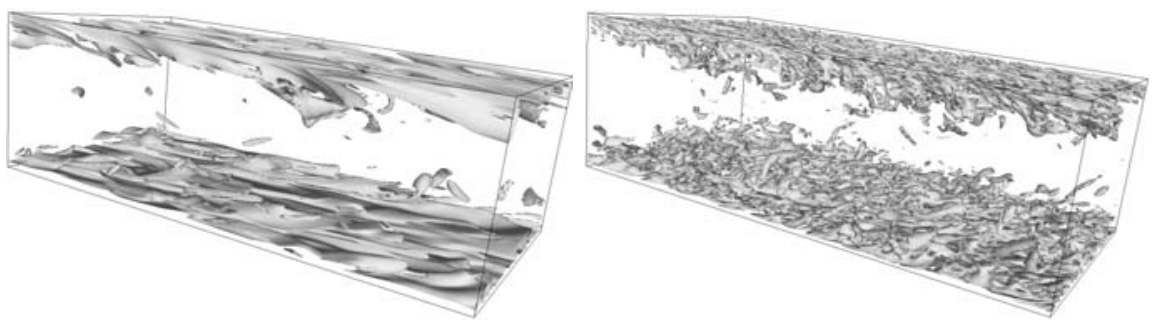

Fig. 13. Turbulent plane channel, vorticity modulus; a) DNS $\left(h^{+}=165\right)$, b) LES using the spectral-dynamic model $\left(h^{+}=389\right)$, from Lamballais [57].

and B. The flow goes from left to right. It is clear that the large-eddy simulation does reproduce features expected from turbulence at higher Reynolds number, and displays much more vortical activity in the small scales that the DNS.

\section{Improved models for LES}

We have clearly shown in the former chapter the advantages of the spectral eddy-viscosity models, with in particular the possibility of accounting for local or semi-local effects in the neighbourhood of the cutoff. However, we have already stressed that in most of industrial or environmental applications, the complexity of the computational domain forbids the use of spectral methods. One has thus to deal with numerical codes written in physical space and employing finite-volume or finite-differences methods, often with unstructured grids. This last point will not be considered here, although it is crucial for practical applications. We will present however simulations on orthogonal grids of mesh size varying in direction and location, sometimes in curvilinear geometry. The present chapter will be devoted to models of the structure-function family, with applications to isotropic turbulence, free-shear flows and boundary layers.

\subsection{Structure-function model}

\subsubsection{Formalism}

The Structure-Function model is an attempt to go beyond Smagorinsky, while keeping in physical space the same scalings as the spectral eddy viscosity. The original Structure-Function (SF) model is due to Métais and Lesieur [80] (see also Lesieur and Métais [69]). It consists in building in physical space an eddy viscosity normalized by $\left.\sqrt{E_{\vec{x}}\left(k_{\mathrm{C}}\right) / k_{\mathrm{C}}}\right)$, still with $k_{\mathrm{C}}=\pi / \Delta x$. The spectrum $\left.E_{\vec{x}}\left(k_{\mathrm{C}}, t\right)\right)$ is a local kinetic-energy spectrum 
at a given point $\vec{x}$, which has to be properly determined assuming that turbulence is locally isotropic. This allows to take into account the spatial intermittency of turbulence. One first discards the peak behaviour ${ }^{3}$ of $K(x)$ in equation (4.14) and adjust the constant as proposed by Leslie and Quarini [66], by balancing in a $k^{-5 / 3}$ inertial range extending from zero to $k_{\mathrm{C}}$ the subgridscale flux $2 \int_{0}^{k_{\mathrm{C}}} \nu_{\mathrm{t}} k^{2} \bar{E}(k) \mathrm{d} k$ with the kinetic energy flux $\epsilon$. This yields

$$
\nu_{\mathrm{t}}\left(k_{\mathrm{C}}\right)=\frac{2}{3} C_{\mathrm{K}}^{-3 / 2}\left[\frac{E\left(k_{\mathrm{C}}\right)}{k_{\mathrm{C}}}\right]^{1 / 2} .
$$

We keep in mind that $E\left(k_{\mathrm{C}}\right)$ is now a local kinetic-energy spectrum, which has to be evaluated in terms of physical-space quantities. The best candidate for that is the second-order velocity structure function

$$
F^{\mathrm{is}}(r)=\left\langle[\vec{u}(\vec{x}, t)-\vec{u}(\vec{x}+\vec{r}, t)]^{2}\right\rangle,
$$

where the label "is" stands for isotropic turbulence, and the brackets correspond to ensemble averaging. The equivalence of Kolmogorov's $\epsilon^{2 / 3} k^{-5 / 3}$ spectrum is the $\left\langle\delta v(r)^{2}\right\rangle \sim(\epsilon r)^{2 / 3}$ structure-function. This was the original formulation of Kolmogorov's law (Kolmogorov [53]). We recall also Batchelor's relation in isotropic turbulence

$$
F^{\mathrm{is}}(\Delta x)=4 \int_{0}^{\infty} E(k, t)\left(1-\frac{\sin (k \Delta x)}{k \Delta x}\right) \mathrm{d} k .
$$

For the subgrid-modelling problem, we consider the following local structure-function

$$
F_{2}(\vec{x}, \Delta x)=\left\langle[\overrightarrow{\vec{u}}(\vec{x}, t)-\overline{\vec{u}}(\vec{x}+\vec{r}, t)]^{2}\right\rangle_{\|\vec{r}\|=\Delta x} .
$$

The difference with relation (5.2) is that $F_{2}$ is calculated with a local statistical average of square (filtered) velocity differences between $\vec{x}$ and the six closest points surrounding $\vec{x}$ on the computational grid. In some cases, the average may be taken over four points parallel to a given plane ${ }^{4}$. The equivalent Batchelor's formula is

$$
F_{2}(\vec{x}, \Delta x)=4 \int_{0}^{k_{\mathrm{C}}} \bar{E}(k, t)\left(1-\frac{\sin (k \Delta x)}{k \Delta x}\right) \mathrm{d} k,
$$

\footnotetext{
${ }^{3}$ It will be shown later on how to reintroduce the peak in terms of hyperviscosity.

${ }^{4}$ In a channel, for instance, the plane is parallel to the boundaries.
} 
since the filtered field has no energy at modes larger than $k_{\mathrm{C}}$. Assuming again a $k^{-5 / 3}$ spectrum extending from zero to $k_{\mathrm{C}}$, one obtains

$$
\nu_{\mathrm{t}}^{\mathrm{SF}}(\vec{x}, \Delta x)=0.105 C_{\mathrm{K}}^{-3 / 2} \Delta x\left[F_{2}(\vec{x}, \Delta x)\right]^{1 / 2} .
$$

In fact, this derivation of the SF model equation is different and simpler than the one proposed in the original paper of Métais and Lesieur [80], and may be found in Ducros [31].

\subsubsection{Non-uniform grids}

Interpolations of equation (5.6) based on Kolmogorov's 2/3 law for the above structure function may be proposed if the computational grid is not regular (but still orthogonal). Let $\Delta c$ be a mean mesh in the three spatial directions ${ }^{5}$. We have (in the six-point formulation)

$$
F_{2}(\vec{x}, \Delta c)=\frac{1}{6} \sum_{i=1}^{3} F_{2}^{(i)}\left(\frac{\Delta c}{\Delta x_{i}}\right)^{2 / 3}
$$

with

$$
F_{2}^{(i)}=\left[\vec{u}(\vec{x})-\vec{u}\left(\vec{x}+\Delta x_{i} \vec{e}_{i}\right)\right]^{2}+\left[\vec{u}(\vec{x})-\vec{u}\left(\vec{x}-\Delta x_{i} \vec{e}_{i}\right)\right]^{2},
$$

where $\vec{e}_{i}$ is the unit vector in direction $\vec{x}_{i}$.

\subsubsection{Structure-function versus Smagorinsky models}

We have found a relation between Smagorinsky's and the structure-function models, by replacing the velocity increments in the latter by first-order spatial derivatives. One finds for the six-point formulation

$$
\nu_{\mathrm{t}}^{\mathrm{SF}} \approx 0.777\left(C_{\mathrm{S}} \Delta x\right)^{2} \sqrt{2 \bar{S}_{i j} \bar{S}_{i j}+\bar{\omega}_{i} \bar{\omega}_{i}},
$$

where $\overline{\vec{\omega}}$ is the vorticity of the filtered field, whereas $C_{\mathrm{S}}$ is Smagorinsky's constant defined by equation (3.22) in terms of Kolmogorov's constant $C_{\mathrm{K}}$. Then the SF model appears, within this crude first-order approximation, to be a combination of Smagorinsky model in a strain and vortical version. Suppose as an example that we are in the stagnation regions between two quasi two-dimensional vortices (in a mixing layer, or a wake, or in a round jet...) when there is a low residual vorticity which is going to be stretched longitudinally. At this initial stage, and since vorticity in the stagnation

\footnotetext{
${ }^{5}$ It may be geometric, or of another type.
} 
region is low, the vortical term will be small in front of the strain term, so that the SF model will be about $80 \%$ less dissipative than Smagorinsky's, which will favour the eventual stretching of longitudinal vortices. On the other hand, SF should be more dissipative than Smagorinsky within the core of the large vortices.

\subsubsection{Isotropic turbulence}

It has been shown in Métais and Lesieur [80] that, for $C_{\mathrm{K}}=1.4$, the SF model gives a quite good $k^{-5 / 3}$ energy spectrum ${ }^{6}$, whereas Smagorinsky ${ }^{7}$ has more like a $k^{-2}$ inertial range.

\subsubsection{SF model, transition and wall flows}

It was at first quite a disapointment to realize that the SF model was, like Smagorinsky's, too dissipative for transition in a boundary layer (yielding again relaminarization) or in a channel. One might have thought that at least the four-point formulation in planes parallel to the wall would have eliminated the effect of the mean shear at the wall on the eddy viscosity. In fact, it turns out that the isotropic relation (5.5) introduces spurius inhomogeneous effects in the eddy-viscosity, which increase the latter, and the SF model is too dissipative for quasi two-dimensional or transitional situations. This is of course a real concern, specially for turbulent boundary layers or channel flows, and has motivated the development of two improved versions of the SF model: the selective structure-function model (SSF), and the filtered structure-function model (FSF), for which turbulence gets rid of large-scale inhomogeneities before the SF model is applied.

\subsection{Selective structure-function model}

In the selective structure-function (SSF) model (David [27]), the eddyviscosity is switched off when the flow is not three-dimensional enough. We need for that a criterion of three-dimensionalization, defined as follows: one considers at a given time the angle between the vorticity vector at a given grid point and the arithmetic mean of vorticity vectors at the six closest neighbouring points (or the four closest points in the four-point formulation). If one carries out LES of isotropic turbulence at a resolution of $32^{3} \sim 64^{3}$, one finds that the p.d.f. peaks for an angle of $20^{0}$, which is thus the most probable value. Then, the eddy viscosity will be cancelled at

\footnotetext{
${ }^{6}$ With a nearly flat compensated $k^{5 / 3} E(k)$ spectrum.

${ }^{7}$ With $C_{\mathrm{S}}$ still given by equation (3.22).
} 
points where this angle is smaller than $20^{\circ}$. We will give various applications of this model to various heated or compressible flows throughout the book.

\subsection{Filtered structure-function model}

\subsubsection{Formalism}

The filtered structure-function model was developed by Ducros ([31], see also Ducros et al. [33]). The filtered field $\bar{u}_{i}$ is now submitted to a high-pass filter $\widetilde{(.)}$ consisting in a Laplacian operator discretized by second-order centered finite differences and iterated three times. We first apply relation (5.5) to the high-pass filtered field

$$
\tilde{F}_{2}(\vec{x}, \Delta x)=4 \int_{0}^{k_{\mathrm{C}}} \tilde{E}(k)\left(1-\frac{\sin (k \Delta x)}{k \Delta x}\right) \mathrm{d} k,
$$

where $\tilde{F}_{2}(\vec{x}, \Delta x)$ is the second-order structure function of the high-pass filtered field $\widetilde{\widetilde{u}}_{i}$, and $\tilde{E}(k)$ its spectrum. This allows (for isotropic turbulence) to relate $\tilde{F}_{2}$ to $\tilde{E}\left(k_{\mathrm{C}}\right)$, and hence to $E\left(k_{\mathrm{C}}\right)$ thanks to the transfer function of the "tilde" operator, determined with the aid of isotropic test fields. Using equation (5.1) yields for the eddy viscosity

$$
\nu_{\mathrm{t}}^{\mathrm{FSF}}(\vec{x}, \Delta x)=0.0014 C_{\mathrm{K}}^{-3 / 2} \Delta x\left[\tilde{F}_{2}(\vec{x}, \Delta x)\right]^{1 / 2} .
$$

A further advantage of the FSF model is that it does not contain adjustable constants. We will show below very satisfactory applications of this model to mixing layers and boundary layer on a flat plate.

\subsection{A test case for the models: The temporal mixing layer}

We present now in Figure 14 a comparison between Smagorinsky's model, the plain spectral plateau-peak model (not dynamic)) and the various structure function models (original, selective and filtered versions). The comparison is carried out in the case of a temporally-growing mixing layer. Pseudospectral numerical methods are here used. We take a three-dimensional initial isotropic perturbation, but the domain contains now only two fundamental longitudinal most-unstable wavelengths, so that no helical pairing develops. Instead, we see two big rollers oscillating in phase ${ }^{8}$, and stretching longitudinal haipins exactly as in the model of Bernal and Roshko [11], with

\footnotetext{
${ }^{8}$ Such a configuration corresponds to "translative instability", from the work of Pierrehumbert and Widnall [93] on secondary instabilities (Floquet-type analysis) of Stuart vortices.
} 


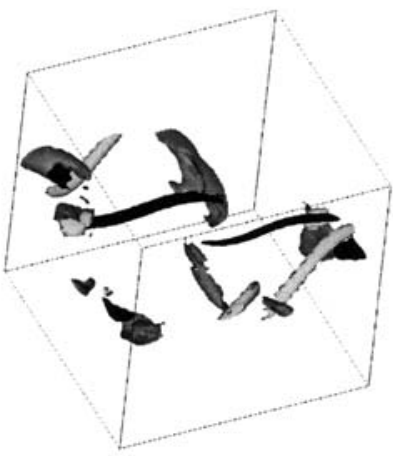

SMAG: $\max \left|\omega_{x}\right|=2.92 \omega_{i}$

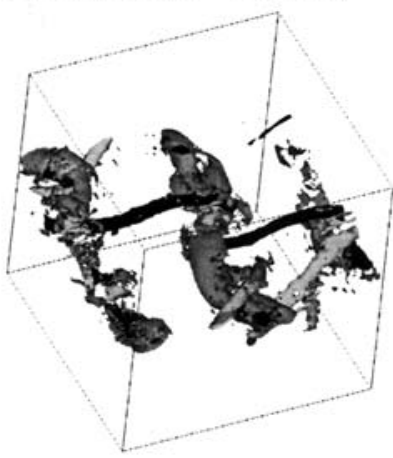

SPEC: $\max \left|\omega_{x}\right|=4.75 \omega_{i}$

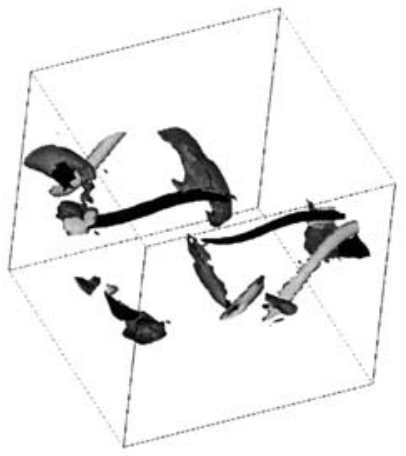

SF: $\max \left|\omega_{x}\right|=2.86 \omega_{i}$

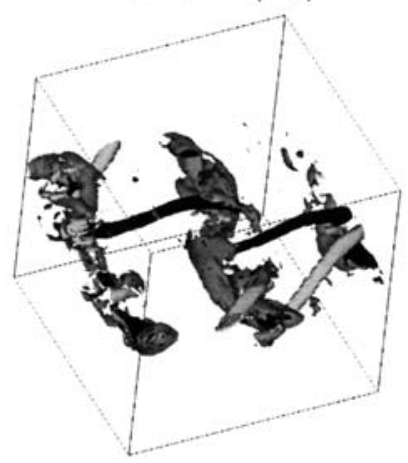

FSF: $\max \left|\omega_{x}\right|=4.83 \omega_{i}$

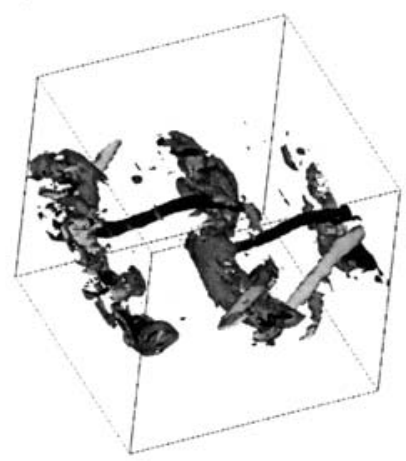

SSF: $\max \left|\omega_{x}\right|=5.42 \omega_{i}$

Fig. 14. Comparison of various SGS models (Smagorinsky, Structure-Function, Plateau-Peak, FSF, SSF) applied to a temporal mixing layer, visualized by isosurfaces $\omega_{x}=\omega_{i}$ (black), $\omega_{x}=-\omega_{i}$ (light grey) and $\omega_{z}=\omega_{i}=-2 U / \delta_{i}$ (dark grey). 
very neat alternate longitudinal vortices. Notice the strong resemblance between the results obtained with the plateau-peak, filtered structure-function and selective structure-function models. They give bigger spanwise and longitudinal vortices than Smagorinsky and the SF models, and much more small-scale variability. This confirms that both modifications of the original structure-function model go in the right direction, since the primary and secondary instabilities are less damped with the new models. Note also that the SF model seems to be here only slightly less dissipative that Smagorinsky's model.

\subsection{Spatially growing mixing layer}

The temporal approximation is only a crude approximation of a mixing layer spatially developing, where one works in a frame traveling with the average velocity between the two layers. We consider now an incompressible mixing layer spatially-developing between two streams of velocity $U_{1}$ and $U_{2}\left(U_{1}>U_{2}\right)$. Further details can be found in Comte et al. [25]. The inflow consists of an hyperbolic-tangent velocity profile

$$
\bar{u}(y)=\frac{U_{1}+U_{2}}{2}+\frac{U_{1}-U_{2}}{2} \tanh \frac{2 y}{\delta_{i}},
$$

where $\delta_{i}$ is the upstream vorticity thickness. The Reynolds number is here built on $\delta_{i}$ and half the velocity difference $U=\left(U_{1}-U_{2}\right) / 2$. A weak random perturbations is superimposed onto the mean profile. The same mixed spectral-compact code already discussed for the channel and the wake is used here. Periodicity is assumed in the spanwise direction $z$. Sine/cosine expansions are used in the transverse direction $y$, enforcing free-slip boundary conditions. Non-reflective outflow boundary conditions are approximated by a multi-dimensional extension of Orlansky's discretization scheme, with limiters on the phase velocity (see Gonze [43] for a detailed description of the numerical code). We first compare a DNS at low Reynolds number $(R e=100)$ with a LES (without molecular viscosity) using the FSF model. The upstream forcing consists here in a quasi-twodimensional random perturbation.

Figure 15 (top) shows an isosurface of the vorticity modulus obtained in the DNS. The vortex sheet undergoes oscillations leading to a first rollup further downstream. Subsequently, various pairings of Kelvin-Helmholtz vortices are observed. Again, thin intense longitudinal vortices are stretched as in Bernal and Roshko's [11] experiment. For the DNS, the vorticity magnitude during the run peaks at $2 \omega_{i}$, where $\omega_{i}=2 U / \delta_{i}$ is the maximal vorticity magnitude introduced at the inlet. Although less computational points are used in the LES, the LES (Fig. 15, bottom) is obviously much more turbulent than the DNS, and has also a lot of oblique waves propagating 

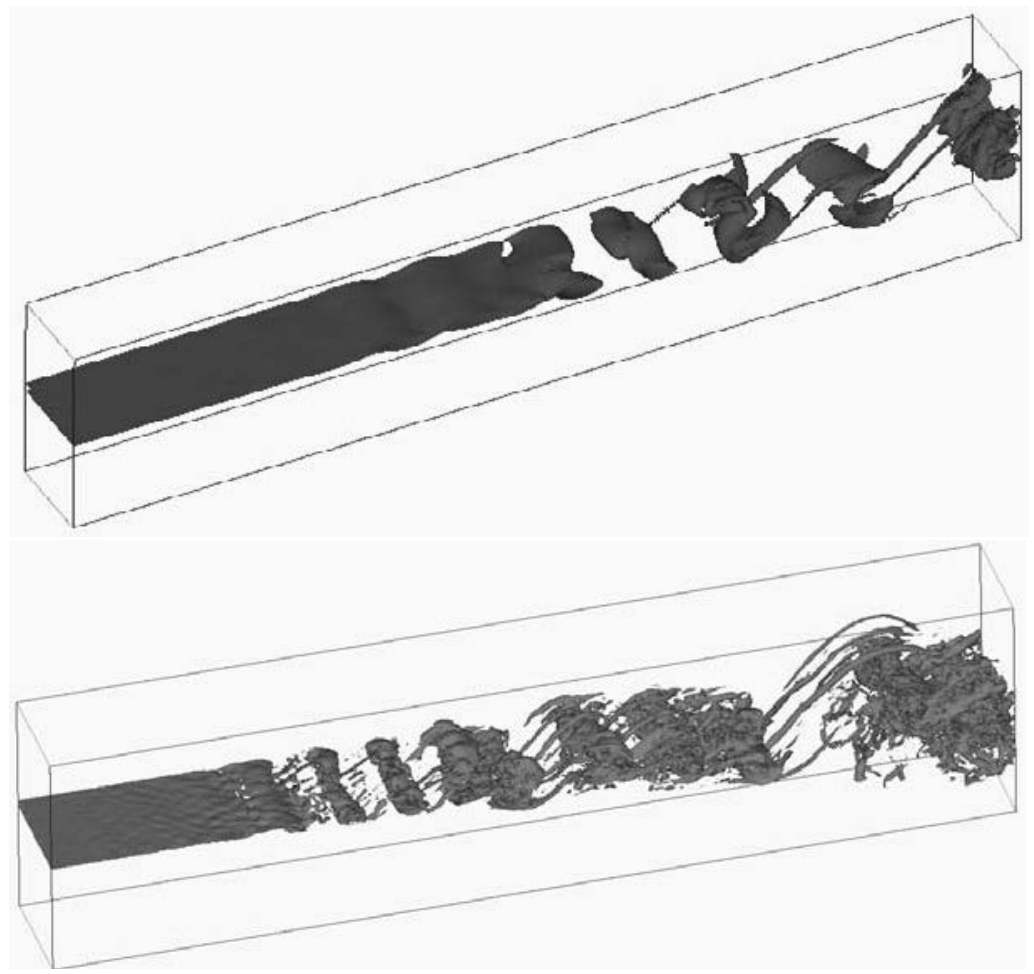

Fig. 15. Perspective views of isovorticity surface: top, DNS, $\|\vec{\omega}\|=\omega_{i} / 3$; bottom, run LES, $\|\omega\|=(2 / 3) \omega_{i}$.

along the upstream vortex sheet. The latter breaks down much faster, and the longitudinal vortices are stretched much more efficiently. Indeed, the maximal vorticity magnitude is now $\approx 4 \omega_{i}$ for the whole run. Roll-up and pairing events occur much faster than in the DNS. Notice the complexity of the dynamics with a cluster of three fundamental Kelvin-Helmholtz vortices undergoing a first pairing and, at its downstream end, a billow made of 4 fundamental $\mathrm{KH}$ vortices whose second pairing is in progress.

Similar simulations but with a domain of spanwise size doubled (still with a quasi-twodimensional random forcing) show that the vortical structure changes quite radically when the spanwise direction is increased. Figure 16, taken from Comte et al. [25], indeed shows respectively the low-pressure and vorticity fields in that case. It is clear at least on the pressure that helical pairing develops, as in the experiments of Browand and Troutt [15]. 

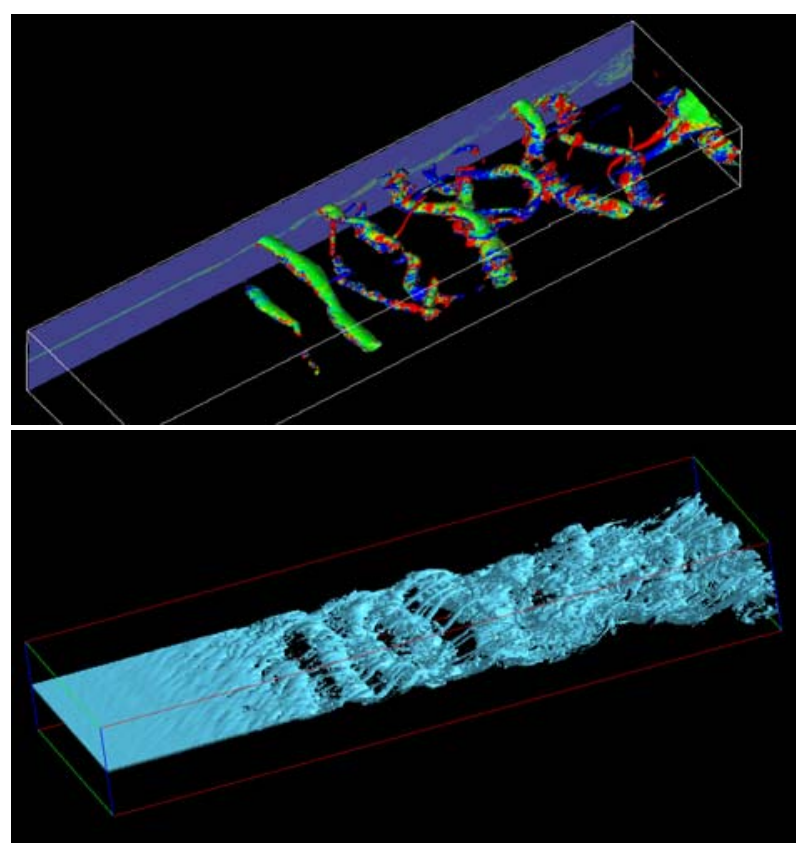

Fig. 16. Wide domain, LES. Top, low pressure; bottom, high vorticity.

\subsection{Vortex control in a round jet}

Our goal here is to demonstrate the ability of the LES to properly reproduce the coherent-vortex dynamics in the transitional region of the jet. We also show the possibility of controlling the jet behaviour by manipulating the inflow conditions. The control of the turbulent jets find numerous industrial applications in thermohydraulics, aeronautics, industrial processes or even the dispersion of pollutants. For these applications, it is particularly interesting to control certain flow characteristics such as the mixing efficiency, the acoustic generation, etc. We will show below that an efficient control requires a precise knowledge of the spatial and temporal flow organization to manipulate the three-dimensional coherent vortices. In the last ten years, the progress in the experimental methods of detection and identification has made possible a detailed investigation of the complex three-dimensional coherent vortices imbedded within this flow. For instance, the influence of the entrainment of the secondary streamwise vortices has been studied by Liepmann and Gharib [74]. On the numerical side, several simulations of two-dimensional or temporally evolving jets have been performed. Very few have however investigated the three-dimensionnal spatial development of the 
round jet. We here show how LES can be used to perform a precise study of the spatial growth of the round jet from the nozzle up to several diameters downstream. Round jets and there control have been numerically studied in details by Urbin [108], Urbin and Métais [109] and Urbin et al. [110]. We here briefly present recent results obtained by Silva [103] with the mixed compact/spectral numerical code previously described. The use of LES techniques allow us to reach high values of the Reynolds number. We here use the Selective Structure Function Model which is well adapted for transitional flows. We consider a computational domain starting at the nozzle and extending up to several jet diameters downstream. We successively consider two jets configurations: the "natural" jet $(R e=25000)$ which is forced upstream by the top-hat profile to which is superposed a weak $3 \mathrm{D}$ white noise; the "excited" jet development $(R e=10000)$ is controlled with the aid of a given deterministic inflow forcing (plus a white noise) designed to trigger a specific type of three-dimensional coherent structures.

Experimental studies by Michalke and Hermann [83] have clearly pointed out the capital effect of the inflow momentum boundary layer thickness $\theta$ and of the ratio $R / \theta$ ( $R$ : jet radius) on the jet downstream development. It was shown that the detailed shape of the mean velocity profile strongly influences the nature of the coherent vortices appearing near the nozzle: either axisymmetric structures (vortex rings) or helical structure can indeed develop. Here, the flow inside the nozzle was not simulated, but a mean axial velocity profile of top-hat shape in accordance with the experimental measurements was imposed:

$$
W(r)=\frac{1}{2} W_{\mathrm{o}}\left[1-\tanh \left(\frac{1}{4} \frac{R}{\theta}\left(\frac{r}{R}-\frac{R}{r}\right)\right)\right]
$$

where $W_{\mathrm{o}}$ is the velocity on the axis. Here, $R / \theta=20$. For such an inlet profile, linear stability analysis predicts a slightly higher amplification rate for the axisymmetric (varicose) mode than for the helical mode (see Michalke and Hermann [83]).

We first consider the "natural" jet which is forced upstream by the top-hat profile given by equation (5.13), to which is superposed a weak three-dimensional white noise. The frequency spectra revealed the emergence of a predominant vortex-shedding Strouhal number (normalized by $D$ and $\left.W_{0}\right), S t r_{\mathrm{D}}=0.375$, in good agreement with the experimental value. The LES shown in Figure 17 shows that the Kelvin-Helmholtz instability along the jet edge yields further downstream vortices having mainly an axisymmetric toroidal shape. One sees in Figure $17 \mathrm{~b}$ that an original vortex arrangement can be observed subsequent to the varicose mode growth: the "alternate pairing". Such a vortex interaction was previously observed by Fouillet [36] and Comte et al. [23] in the DNS of a temporally evolving 
round jet at low Reynolds number $(R e=2000)$. The direction normal to the toroidal vortices symmetry plane tends, during their advection downstream, to differ from the jet axis. The inclination angle of two consecutive vortices appears to be of opposite sign, eventually leading to local vortex reconnections with an alternate arrangement. Note that vortex loop's inclination at the end of the potential core was experimentally observed by Petersen [92]. Experimental evidence of "alternate pairing" was given by Broze and Hussain [16]. This alternate-pairing mode corresponds to the growth of a subharmonic perturbation (of wavelength double of the one corresponding to the rings) developing after the formation of the primary rings. It therefore presents strong analogies with the helical-pairing mode observed in plane mixing layers (see above).

We here now show how a deterministic inflow perturbation can trigger one particular flow organization. We apply a periodic fluctuation associated with a frequency corresponding to $S t r_{\mathrm{D}}=0.375$ for which the jet response is known to be maximal. The inflow excitation is here chosen such that alternate-pairing mode previously described is preferentially amplified. The resulting structures are analogous to Figure 17 except that the alternatively inclined vortex rings now appear from the nozzle (see Fig. 18). One of the striking features is the very different spreading rates in different directions. Note that the present jet exhibits strong similarities with the "bifurcating" jet of Lee and Reynolds [63]. One of the important technological application of this peculiar excitation resides in the ability to polarize the jet in a preferential direction.

\subsection{LES of spatially developing boundary layers}

The standard Structure-Function model permits to go beyond transition in a temporal (periodic in the flow direction) compressible boundary layer upon an adiabatic wall at Mach 4.5 (see Ducros et al. [32]). But it does not work for transition in a boundary layer at low Mach (or incompressible) where, like Smagorinsky, it is too dissipative and prevents small perturbations to degenerate into turbulence. Conversely, it has been used with success in its filtered version (FSF model) for the simulation of a quasi-incompressible $\left(M_{\infty}=0.5\right)$ boundary layer of an ideal gas developing spatially over an adiabiatic flat plate with a low level of upstream forcing (Ducros et al. [33]). Although it gives interesting qualitative information on the structure of turbulent boundary layers, the above LES did not have a sufficient resolution close to the wall (first point at $y^{+}=5 \approx 6$ ) for good predictions of average quantities such as the friction coefficient at the wall or the shape factor. Here, we present new results with a finer resolution at the wall $\left(y^{+}=1\right.$ or 2$)$, at a lower Mach number (0.3). The computations are performed with the COMPRESS numerical code briefly described in Section 10. 


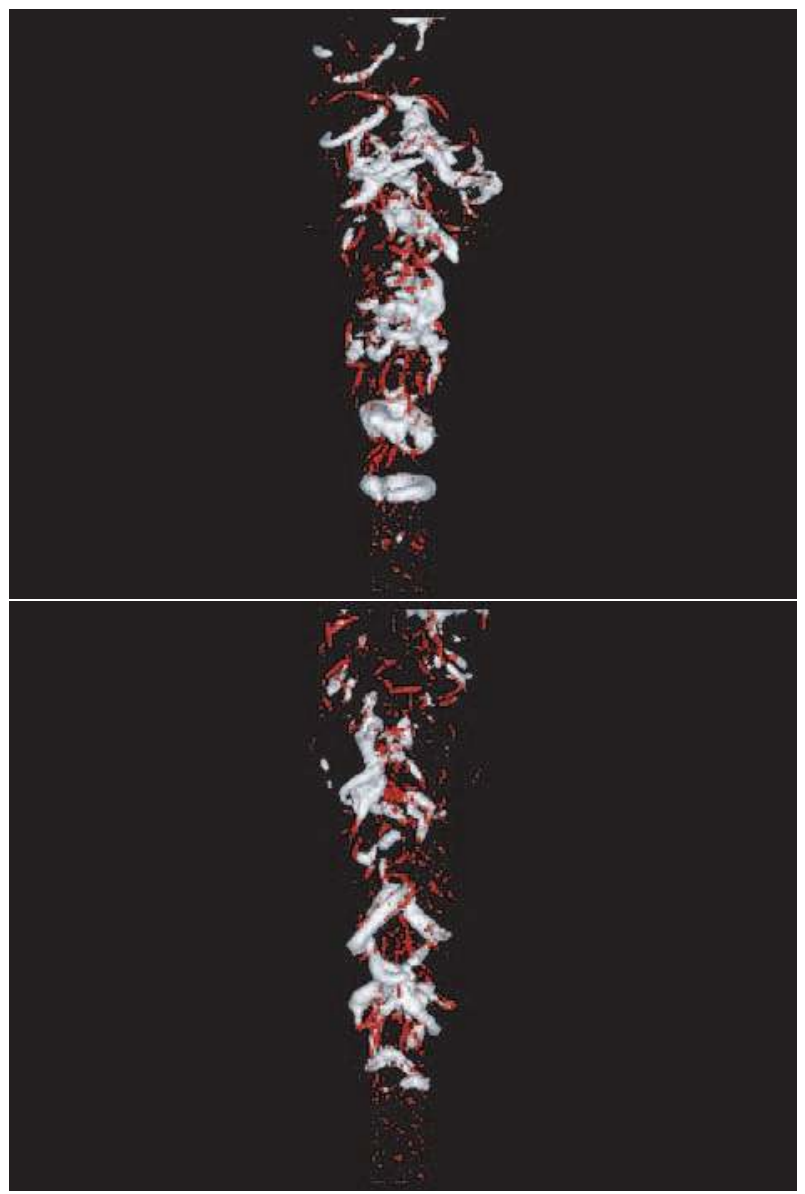

Fig. 17. LES of the natural jet at $R e=25000$ : instantaneous visualizations at two different times. White: low pressure isosurface; dark: regions of $Q>0$ (courtesy Silva).

The details of this computations are presented in Briand [14]. It is known that transition in the boundary layer on a flat plate depends upon the type of perturbations exerted upstream on the flow (see Lesieur [67]). In Klebanoff et al. [51], the boundary layer was forced upstream with a thin metal ribbon parallel to the wall and stretched in the spanwise direction, which vibrates two-dimensionally close to the wall. In this experiment, the $3 \mathrm{D}$ forcing was harmonic. This corresponds to what is referred to as the $\mathrm{K}$-mode, where the crests of the TS waves oscillate in phase in the spanwise 

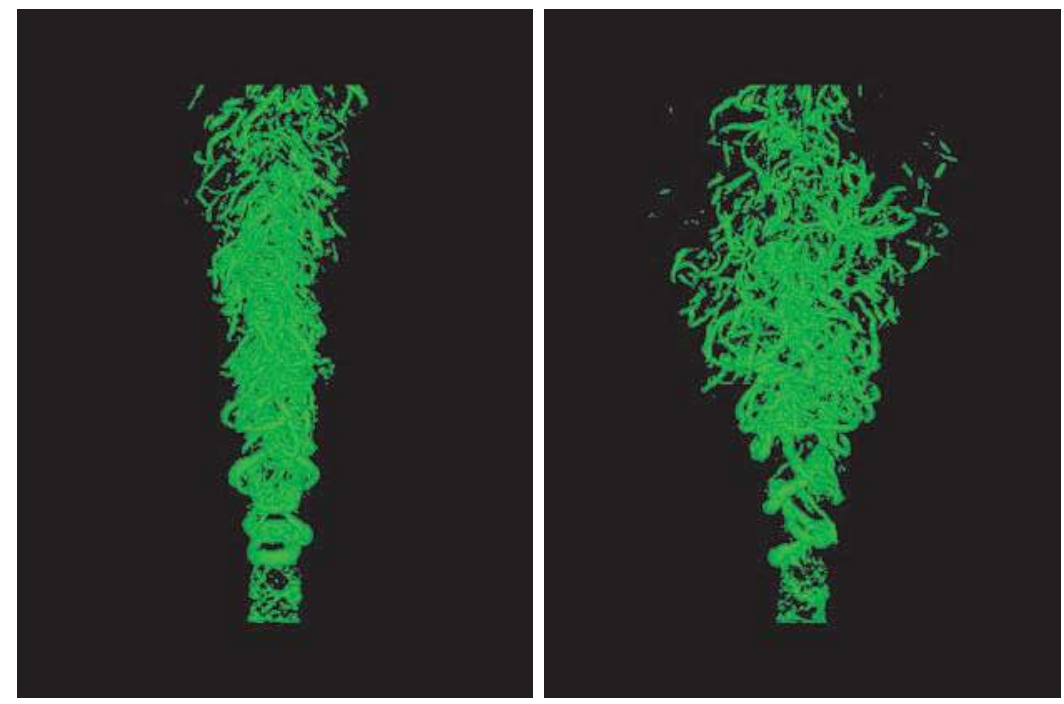

Fig. 18. Bifurcation of the jet with alternate-pairing excitation: forced jet at $R e=10000$. Instantaneous vizualisations of regions of $Q>0$ at the same instant but seen from two different perpendicular directions (courtesy Silva).

direction. The resulting vortex filaments are therefore aligned in the flow direction. On the other hand, if the perturbation is subharmonic, the crests oscillate out of phase. This is called H-mode, from Herbert [45], and corresponds to a staggered organization of vortex filaments. Herbert could show for the temporal problem $^{9}$, that the staggered mode was more amplified than the aligned mode. This should favour the emergence of H-mode during transition in natural situations, and explain why the transition above seems to be of subharmonic type.

We now return to the LES of the spatially-developing boundary layer over a flat plate. It is started with a set of upstream conditions (harmonic $\mathrm{K}$-mode or subharmonic H-mode) obtained with the aid of nonlinear parabolized stability expansion (PSE) calculations (Bertolotti and Herbert [12], Airiau [5]). To the upstream state corresponding with a Reynolds number $R_{\delta_{i}}=1000$ ( $\delta_{i}$ being the upstream displacement thickness), one superposes a $3 \mathrm{D}$ white-noise of amplitude 0.2 the amplitude of the PSE perturbation. In the $\mathrm{K}$-case, one sees in the transitional region formation of big longitudinal $\Lambda$-shaped vortices lying on the wall, and in phase in the spanwise

\footnotetext{
${ }^{9}$ Using a secondary-instability analysis where a perturbation is superposed on a TS wave of finite amplitude.
} 

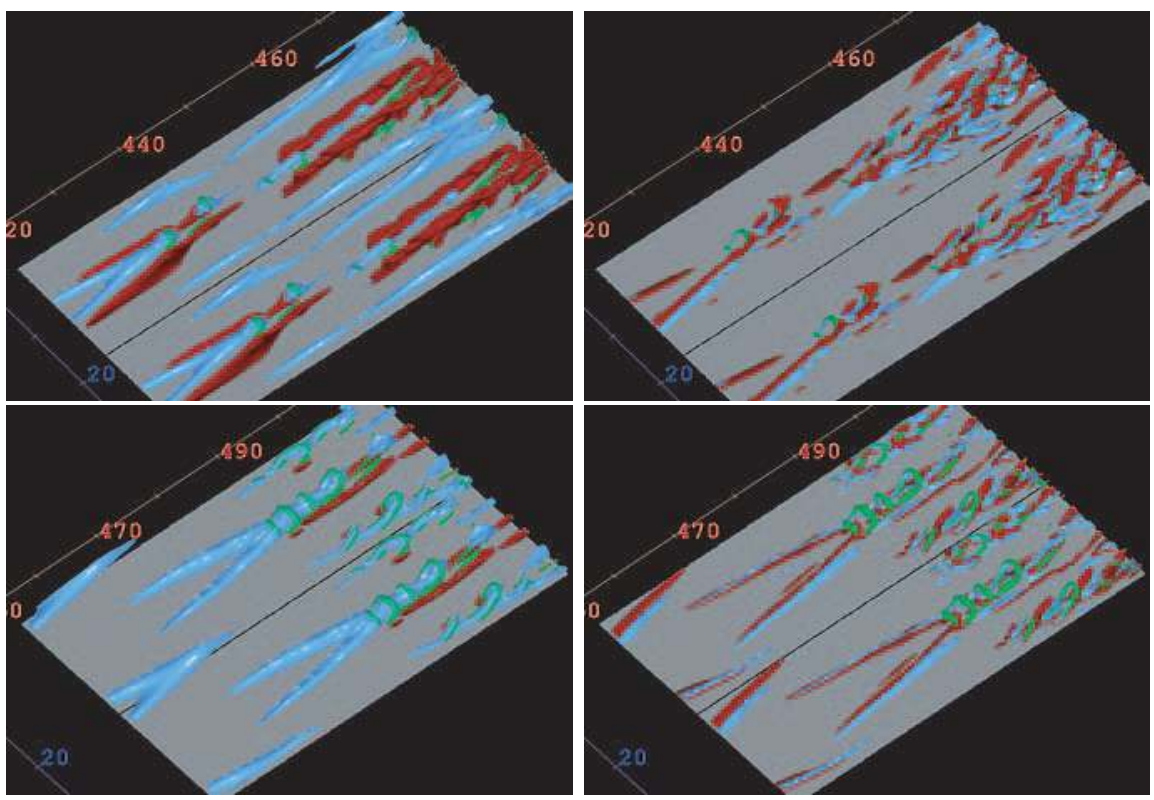

Fig. 19. LES of a spatial boundary layer at Mach 0.3 ; top and bottom, K- and H-transition respectively; the l.h.s. and r.h.s. correspond respectively to velocity and vorticity fluctuation components (dark, positive, light grey, negative); dark grey marks isosurfaces of positive $Q$ (courtesy Briand).

direction (see Fig. 19, top). In the H-case, the vortices are staggered (see Fig. 19, bottom). The figures show at the end of transition the longitudinal components of velocity and vorticity, and also positive $Q$. One sees that the $\Lambda$ vortices are very well correlated with a system of induced high and lowspeed streaks ${ }^{10}$. Remark also on the vorticity plots that the big $\Lambda$ 's induce "antivorticity" close to the wall, due to the zero velocity condition at the wall. Downstream of $\approx 440 \delta_{i}$, the streaks become purely longitudinal. This is accompanied by the fast shedding of small arch vortices ejected from the tip of the $\Lambda$ 's, as indicated by $Q$-isosurfaces.

Figure 20 shows for the K-transition the downstream evolution of the friction coefficient at the wall, with comparison against the theoretical predictions of Van Driest ${ }^{11}$ and Barenblatt and Prostokishin [7]. One sees a good agreement of the LES with these predictions, a resolution of $y^{+}=1$ improving the result. It is even better in the H-case. The peak in the

\footnotetext{
${ }^{10}$ This is not apparent on the figure for the H-case, due to an ill-chosen threshold.

${ }^{11}$ Discussed in Cousteix [26].
} 


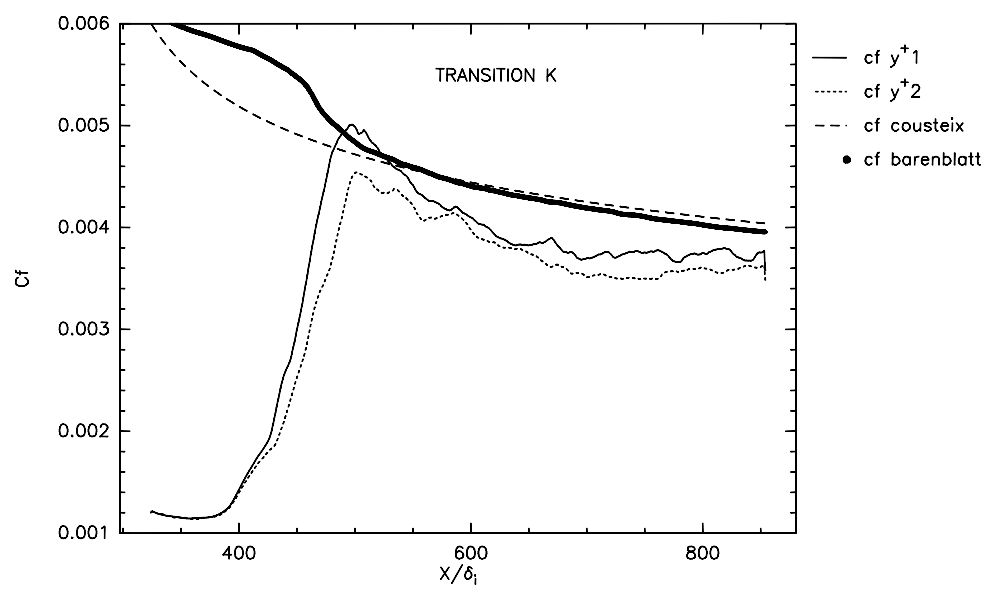

Fig. 20. LES of a spatial boundary layer at Mach 0.3: friction coefficients against downstream distance, compared with theoretical predictions of Cousteix and Barenblatt (courtesy Briand).

friction coefficient is at $490 \delta_{i}$, much further than the change of regime of the velocity streaks, and might be associated to an event such as the localized creation of a big hairpin vortex observed in the simulations of Ducros et al. [33]. Figure 21 shows for the K-case (but results are very close in the H-case) the rms longitudinal velocity component $u^{\prime}$ at a downstream distance such that $R_{\delta_{1}}=1670$ ( $\delta_{1}$ local displacement thickness), compared with Spalart's [106] DNS at Reynolds numbers of 1000 and 2000. Again, the agreement is good, since the results are inbetween Spalart's predictions. If one looks at developed turbulence further downstream, various plots of vorticity components and pressure, as well as $Q$, accompanied with animations, show the very long longitudinal velocity streaks (about 1000 wall units for the low speeds). Above these streaks are ejected hairpins through what resembles a secondary Kelvin-Helmholtz instability occuring at a height of about $30 \approx 40$ wall units. The hairpins first creep at the wall, then rise, due to self-induction effects. Their length is about 300 wall units, so that there are several hairpins (about 3 ) above a single low-speed streak. In this sense, we have no more the perfect correlation hairpins-streaks which we observed during the transitional stage. It is therefore difficult to associate in the developed region the streaks to a system of purely longitudinal alternate vortices at the wall. 


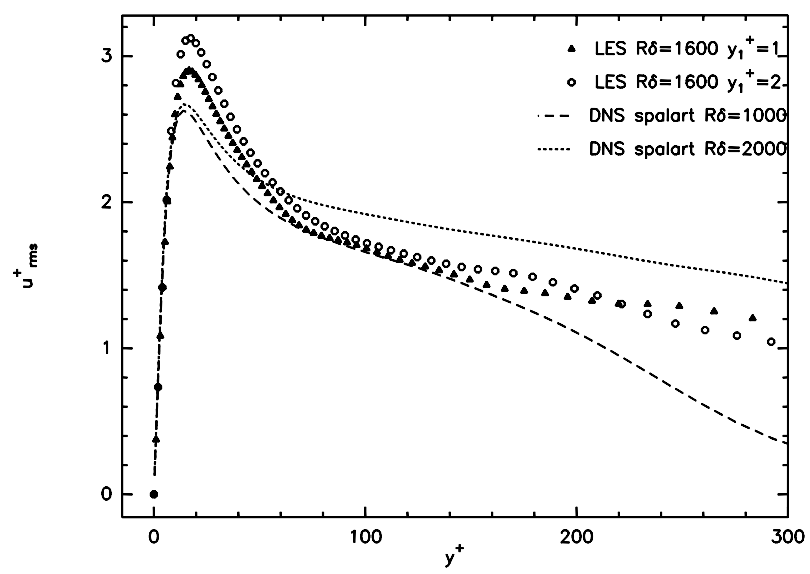

Fig. 21. Spatial boundary layer at Mach 0.3 ; rms velocity fluctuations compared with Spalart's DNS (courtesy Briand).

\section{Dynamic approach in physical space}

\subsection{Dynamic models}

We have shown already in Section 4.1 how a valuable information concerning a given eddy viscosity (here the spectral plateau-peak eddy viscosity) could be obtained thanks to a double filtering through the cutoff $k_{\mathrm{C}}$ and a fictitious cutoff $k_{\mathrm{C}}^{\prime}=k_{\mathrm{C}} / 2$, and the calculation of explicit resolved transfers across $k_{\mathrm{C}}^{\prime}$. This is the underlying philosophy of the dynamic model in physical space (Germano [41]). The basic method applies to a LES using an eddy-viscosity model. Most of the historical developments have been done with Smagorinsky's model, but the procedure may be applied to any eddyviscosity models. One starts with a regular LES corresponding to a filter bar of width $\Delta x$, operator associating to a function ${ }^{12} f(\vec{x}, t)$ the function $\bar{f}(\vec{x}, t)$. One then defines a second "test filter" tilde of larger width $\alpha \Delta x$ (for instance $\alpha=2$ ), associating $\tilde{f}(\vec{x}, t)$ to $f(\vec{x}, t)$. We then have two filter operators bar and tilde which apply on the functions, the product of them ${ }^{13}$ being tilde o bar. This product, applied to $f(\vec{x}, t)$, means that we apply first to $f$ the bar filter (to yield $\bar{f}$ ) then the tilde filter to obtain $\widetilde{\bar{f}}$. Let us first apply this filter product to the Navier-Stokes equation (with constant density): the subgrid-scale tensor of the field $\widetilde{\bar{u}_{i}}$ is readily obtained from

\footnotetext{
${ }^{12}$ Scalar, or vector, or tensor.

${ }^{13}$ In the sense of product of operators.
} 
equation (3.5) with the replacement of the filter bar by the double filter, that is:

$$
\mathcal{T}_{i j}=\widetilde{\tilde{u}}_{i} \tilde{\bar{u}}_{j}-\widetilde{\overline{u_{i} u_{j}}} .
$$

We consider now the field $\bar{u}_{i}$ per se ${ }^{14}$, and evaluate the resolved turbulent stresses obtained by application of the tilde filter. They write:

$$
\mathcal{L}_{i j}=\widetilde{\bar{u}}_{i} \widetilde{\bar{u}}_{j}-\widetilde{\bar{u}_{i} \bar{u}_{j}} .
$$

We now apply the filter "tilde" to equation (3.5), which leads to

$$
\widetilde{T_{i j}}=\widetilde{\overline{u_{i} \bar{u}_{j}}}-\widetilde{\widetilde{u_{i} u_{j}}}
$$

Adding equations (6.2) and (6.3), yields, with the aid of (6.1)

$$
\mathcal{L}_{i j}=\mathcal{T}_{i j}-\widetilde{T_{i j}}
$$

This expression is called Germano's identity. In the r.h.s., $\mathcal{T}_{i j}$ and $\widetilde{T_{i j}}$ have to be modelled, while the l.h.s. $\mathcal{L}_{i j}$ (the resolved stresses) can be explicitly calculated by applying the tilde filter to $\bar{u}_{i}$.

We use Smagorinsky's model expression defined by equation (3.21) and "tilde-filter" it, to get

$$
\widetilde{T_{i j}}-\frac{1}{3} \widetilde{T_{l l}} \delta_{i j}=2 \widetilde{\mathcal{A}_{i j} C},
$$

whith $C=C_{\mathrm{S}}^{2}$ and

$$
\mathcal{A}_{i j}=(\Delta x)^{2}|\bar{S}| \bar{S}_{i j} .
$$

We have now to determine $\mathcal{T}_{i j}$, stress resulting from the filter product. This is again obtained using Smagorinsky for the largest filter, which yields

$$
\mathcal{T}_{i j}-\frac{1}{3} \mathcal{T}_{l l} \delta_{i j}=2 \mathcal{B}_{i j}
$$

whith

$$
\mathcal{B}_{i j}=\alpha^{2}(\Delta x)^{2} \mid \widetilde{\bar{S}}_{\mid} \widetilde{\bar{S}}_{i j}
$$

Substracting equation (6.5) from equation (6.6) yields with the aid of Germano's identity

$$
\mathcal{L}_{i j}-\frac{1}{3} \mathcal{L}_{l l} \delta_{i j}=2 \mathcal{B}_{i j} C-2 \widetilde{\mathcal{A}_{i j} C} .
$$

\footnotetext{
${ }^{14} \mathrm{As}$ if it were the instantaneous field.
} 
This is a nice result relating the model coefficient (unknown) to the resolved stresses. However there are some difficulties. First, one removes $C$ from the filtering as if it were constant ${ }^{15}$, leading to

$$
\mathcal{L}_{i j}-\frac{1}{3} \mathcal{L}_{l l} \delta_{i j}=2 C M_{i j},
$$

with

$$
M_{i j}=\mathcal{B}_{i j}-\widetilde{\mathcal{A}_{i j}} .
$$

All the terms of equation (6.7) may now be determined with the aid of $\bar{u}$. But there are five independent equations for only one variable $C$, so that the problem is overdetermined. A first solution proposed by Germano [41] is to multiply tensorially equation (6.7) by $\bar{S}_{i j}$, to get

$$
C=\frac{1}{2} \frac{\mathcal{L}_{i j} \bar{S}_{i j}}{M_{i j} \bar{S}_{i j}}
$$

(indeed, and due to incompressibility, $\bar{S}_{i i}=0$ ). This provides finally a dynamical evaluation of $C(\vec{x}, t)$, which can be used in the LES of the bar field $\bar{u}$. But problems still arise: in tests using channel flow data obtained from DNS, Germano [41] could show that the denominator in equation (6.8) could locally vanish or become sufficiently small to yield computational instabilities. To get rid of this problem, Lilly [76] chose to determine the value of $C$ in equation (6.7) by minimizing the error using a least squares approach, which gives

$$
C=\frac{1}{2} \frac{\mathcal{L}_{i j} M_{i j}}{M_{i j}^{2}}
$$

and removes the undeterminacy of equation (6.7). However, the analysis of DNS data reveals that the $C$ field predicted by the models (6.8) or (6.9) varies strongly in space and contains a significant fraction of negative values, with a variance which may be ten times higher than the square mean. So, the removal of $C$ from the filtering operation is not really justified and the model exhibits some mathematical inconsistencies. The possibility of negative $C$ is an advantage of the model since it allows a sort of backscatter in physical space, but very large negative values of the eddy viscosity is a destabilizing process in a numerical simulation, yielding a non-physical growth of the resolved scale energy. The cure which is often adopted to avoid excessively large values of $C$ consists in averaging the numerators and denominators of (6.8) and (6.9) over space and/or time, thereby losing some of the

\footnotetext{
${ }^{15}$ This is in some way contradictory with the original aim of having a dynamic evaluation of $C$ depending on space and time.
} 
conceptual advantages of the "dynamic" local formulation. Averaging over direction of flow homogeneity has been a popular choice, and good results have been obtained by Germano [42] and Piomelli [94], who took averages in planes parallel to the walls in their channel-flow simulation. Remark that the same thing has been done, with success, when averaging the dynamic spectral eddy viscosity in the channel-flow LES presented above. It can be shown that the dynamic model gives a zero subgrid-scale stress at the wall, where $L_{i j}$ vanishes, which is a great advantage with respect to the original Smagorinsky model; it gives also the proper asymptotic behavior near the wall.

As already stressed, the use of Smagorinsky's model for the dynamic procedure is not compulsory, and any of the models described in the present paper can be a candidate. As an example, El Hady and Zang [34] have applied the dynamic structure-function model applied to a compressible boundary layer above a long cylinder.

\section{Alternative models}

\subsection{Generalized hyperviscosities}

One of the drawback of the structure-function model given by equation (5.6) is the absence of a cusp near $k_{\mathrm{C}}$. However, EDQNM data show that the exponential form given in equation (4.14) can be correctly approximated by a power law of the type:

$$
\nu_{\mathrm{t}}^{*}\left(\frac{k}{k_{\mathrm{C}}}\right)=\left(1 .+\nu_{\mathrm{t} n}^{*}\left(\frac{k}{k_{\mathrm{C}}}\right)^{2 n}\right),
$$

with $2 n \approx 3.7$. Lesieur and Métais [69] have shown that $\nu_{\mathrm{t} n}^{*}$ can be determined by considering the energy balance between explicit and subgrid-scale transfers. This yields:

$$
\nu_{\mathrm{t} n}^{*}=0.512\left(\frac{3 n}{2}+1\right) .
$$

In fact, the EDQNM value of $2 n=3.7$ is not so far from the exponent $2 n=4$ which would be obtained with a Laplacian operator iterated twice. Therefore, Lesieur and Métais [69] proposed a physical-space turbulent dissipative operator based upon the structure-function model and taking into account the "cusp" behaviour:

$$
2 \frac{\partial}{\partial x_{j}}\left[\nu_{\mathrm{t}}^{\mathrm{SF}} \bar{S}_{i j}\right]+\nu_{\mathrm{t}}^{(2)}\left(\frac{\Delta x}{\pi}\right)^{4}\left(\frac{\partial^{2}}{\partial x_{j}^{2}}\right)^{3} \bar{u}_{i},
$$


where $\bar{S}_{i j}$ is the deformation tensor of the field $\bar{u}_{i} \cdot\left(\partial^{2} / \partial x_{j}^{2}\right)^{3}$ designates the Laplacian operator iterated three-times. $\nu_{\mathrm{t}}^{\mathrm{SF}}$ is given by the r.h.s. of equation (5.6) multiplied by $0.441 /(2 / 3), \nu_{\mathrm{t}}^{(2)}=\nu_{\mathrm{t}}^{\mathrm{SF}} \times \nu_{\mathrm{t} 2}^{*}$, and $\nu_{\mathrm{t} 2}^{*}$ is given by equation (7.2) with $n=2$. The expression (7.3) is interesting in the sense that it provides an eddy dissipation combining the structure function model with a hyperviscosity $\left(\nabla^{2}\right)^{3} \bar{u}_{i}$. The latter represents in physical space the action of the cusp in Kraichnan's spectral eddy viscosity.

\subsection{Hyperviscosity}

The model given by equation (7.3) bears some resemblance to hyperviscosity models which are widely used in the study of geophysical flows because of their simplicity. Indeed, the hyperviscosity consists in replacing the molecular dissipative operator $\nu \nabla^{2}$ by $(-1)^{\alpha-1} \nu_{\alpha}\left(\nabla^{2}\right)^{\alpha}$, where $\alpha$ is a positive integer. As opposed to equation (7.3), $\nu_{\alpha}$ is here a constant (positive) coefficient which has to be adjusted. This has been widely used in two-dimensional isotropic turbulence (see [10]), with $\alpha=2$ or $\alpha=8$, as a way to shift the dissipation to the neighbourhood of $k_{\mathrm{C}}$. This allows for a reduction of the number of scales strongly affected by viscous effects, and has rendered possible in the case of two-dimensional turbulence to demonstrate the existence of coherent vortices.

In three-dimensional turbulence, it was used by Bartello et al. [9] to study the influence of a solid-body rotation, with surprisingly good results.

\subsection{Scale-similarity and mixed models}

The lack of correlation between the subgrid-scale stress and the large-scale strain rate tensors has led Bardina et al. [8] to propose an alternative subgrid-scale model called the scale similarity model. This is based upon a double filtering approach and on the idea that the important interactions between the resolved and unresolved scales involve the smallest eddies of the former and the largest eddies of the latter. They suggest that the real subgrid tensor is similar to the stress tensor constructed from the resolved velocity field. One then writes:

$$
T_{i j}=\overline{\bar{u}}_{i} \overline{\bar{u}}_{j}-\overline{\bar{u}_{i} \bar{u}_{j}} .
$$

The analysis of DNS and experimental data $[8,77]$ have shown that the modelled subgrid-scale stress deduced from (7.4) exhibits a good correlation with the real (measured) stress. However, when implemented in LES calculations, the model hardly dissipates any energy. It is therefore necessary to combine it with an eddy-viscosity type model such as Smagorinsky's model to produce the "mixed" model (see e.g. Meneveau and Katz [82]). 
In the line of Bardina et al. model, new formulations have been proposed to correct this lack of dissipation. Liu et al. [77] have proposed the following model:

$$
T_{i j}=C_{\mathrm{L}}\left(\tilde{\bar{u}}_{i} \tilde{\bar{u}}_{j}-\widetilde{\bar{u}_{i} \bar{u}_{j}}\right),
$$

where $C_{\mathrm{L}}$ is a dimensionless coefficient. The operator $\sim$ consists in a second filter of different width [77].

\subsection{Anisotropic subgrid-scale models}

As stressed above, the subgrid-scale tensor and buoyancy flux given by (3.14) are assumed to be strictly proportional to the grid-scale strain rate tensor and buoyancy flux, respectively. Abbà et al. [1] have proposed an anisotropic formulation of (3.14) using eddy-viscosity and eddy-diffusivity tensors instead of scalar ones:

$$
T_{i j}-\frac{1}{3} T_{l l} \delta_{i j}=2 \sum_{r, s} \nu_{i j r s}^{\mathrm{t}} \bar{S}_{r s}-\frac{2}{3} \delta_{i j} \sum_{l, r, s,} \nu_{l l r s}^{\mathrm{t}} \bar{S}_{r s} .
$$

This formulation allows for a better description of the small-scale anisotropy. This model in conjunction with the dynamic procedure previously described has been used successfully in Large-Eddy Simulations of turbulent natural convection [1]. The reader is referred to the book by Sagaut [98] for a presentation of other anisotropic models.

\section{LES of rotating flows}

Our purpose here is to show the ability of LES and DNS to accurately reproduce the detailed vorticity dynamics and flow statistics even in the presence of external forces like solid-body rotation. Rotating flows are extremely important in engineering for studies related to turbo-machinery of turbines, pumps, or air-intakes of jet engines. They are also crucial in internal geophysics ${ }^{16}$, oceanography, meteorology, planetary or stellar physics. We will first consider rotating shear flows (free or wall bounded), where the effects of rotation are extremely spectacular in terms of modification of the vortical structure and of the statistics. Then we will review studies concerning homogeneous turbulence submitted to rotation.

\footnotetext{
${ }^{16}$ To understand the Earth magnetic-field generation.
} 


\subsection{Rotating shear flows}

We assume a purely incompressible flow submitted to solid-body rotation. We consider a parallel basic (or mean ${ }^{17}$ ) velocity $\langle u\rangle(y)$, and assume that the axis of rotation is parallel to the spanwise direction, such as for the channel of Figure 8 We work in a relative rotating frame of angular-rotation vector $\vec{\Omega}$. Coriolis acceleration $-2 \vec{\Omega} \times \vec{u}$ is added to Navier-Stokes equations, while centrifugal effects are incorporated in the pressure gradient. Let

$$
R_{\mathrm{o}}(y, t)=-\frac{1}{f} \frac{\mathrm{d}\langle u\rangle}{\mathrm{d} y}
$$

(with $f=2 \Omega$ ) be the local Rossby number. It characterizes the ratio of the local relative basic vorticity upon the entrainment vorticity $f$ associated to the solid-body rotation. Regions with a positive (resp. negative) local Rossby will be called cyclonic (resp. anticyclonic). We recall also that the absolute vorticity vector is $\vec{\omega}_{\mathrm{a}}=\vec{\omega}+f \vec{z}$, and satisfies Helmholtz theorem in its conditions of applicability, within which absolute-vortex elements are material.

\subsubsection{Free-shear flows}

We synthesize first results concerning free-shear layers coming both from 3D linear-stability studies (Yanase et al. [112]), and DNS or LES (Lesieur et al. [73], Métais et al. [78]). As in instability studies, we start with a basic parallel velocity profile, weakly perturbed. There is a critical local Rossby number of -1 such that:

- in regions where initially $R_{\mathrm{o}}(y) \geq-1$, the shear layer is twodimensionalized. In a mixing layer for instance, 3D perturbations are damped, and straight Kelvin-Helmholtz billows form ${ }^{18}$. This result agrees in particular with Proudman-Taylor's theorem when the Rossby number modulus is small;

- for $R_{\mathrm{o}}^{\min }<R_{\mathrm{o}}(y)<-1$ ("weak" anticyclonic rotation), where $R_{\mathrm{o}}^{\min } \approx-10 \sim-20$ decreases as the Reynolds increases, the flow is highly three-dimensionalized, with production of intense Görtlerlike alternate longitudinal rolls. This flow three-diemnsionalization results from the development of the so-called "Shear-Coriolis instability". Examination of the vorticity fields shows that they correspond in fact to the condensation of absolute-vortex lines into very long

\footnotetext{
${ }^{17}$ In the sense of an ensemble average.

${ }^{18}$ Without stretching of longitudinal vortices nor helical pairing.
} 
hairpins which are oriented in a purely longitudinal direction. As a result, their spanwise vorticity component is zero, which implies that the mean velocity gradient becomes constant and equal to $f$, so that the local Rossby number uniformizes to the value -1 . This has been clearly shown in the mixing layer DNS by Métais et al. [78] as well as in the anticyclonic region of a wake. There is in fact universality of this result for all shear flows (free or wall bounded), since this law is also found for the channel as shown below.

\subsubsection{Wall flows}

As already stressed, and due to their numerous applications in turbomachinery and also in oceanography, the turbulent flow in a rotating channel of spanwise rotation axis has been subject to extensive studies. Experimentally, it is not easy to cover a wide range of rotation regimes. Conversely, the introduction of the Coriolis acceleration is rather straightforward in numerical codes simulating the three-dimensional Navier-Stokes equations. This may explain why more numerous numerical studies based either on DNS (see e.g. Kristoffersen and Andersson [55]) or LES (see e.g. Kim [50], Miyake and Kajishima [84], Tafti and Vanka [107], and Piomelli and Liu [96]) have been devoted to this topic than experimental ones (see e.g. Johnston et al. [48], Nakabayashi and Kitoh [86]). These previous works have mainly focussed on weak-rotation regimes, and the analysis restricted to statistics directly issued from the velocity field, or studies of the large-scale flow organization. We here recall the main results obatined by Lamballais et al. [57,61]. We precise again the notations. $\vec{\Omega}$ is oriented along the spanwise direction $z$, and may be positive or negative. For the channel flow, the vorticity vector associated with the mean velocity profile $\langle\vec{\omega}\rangle=(0,0,-\mathrm{d}\langle u\rangle / \mathrm{d} y))$ is parallel to $\vec{\Omega}$ near one wall and antiparallel near the opposite wall: we refer to the two particular walls as cyclonic and anticyclonic. Various other terms are currently used. The names suction and pressure sides originate from the pressure gradient due to the Coriolis force, and the terms trailing and leading sides are borrowed from turbo-machinery. The initial Rossby number (already defined above for the channel) turns out to be equal to $R o_{\mathrm{g}}=\frac{3 U_{\mathrm{m}}}{2 \Omega h}$ where $U_{\mathrm{m}}$ is the bulk velocity.

The previous studies have clearly shown that, due to the action of moderate rotation, the flow becomes very asymmetric with respect to the channel center, with a turbulent activity much reduced on the cyclonic side as compared with the anticyclonic side.

We first show a LES of a rotating channel flow based upon the spectraldynamic model with the same characteristic parameters than Piomelli and Liu [96]) in their LES using a localized version of the dynamic model: 


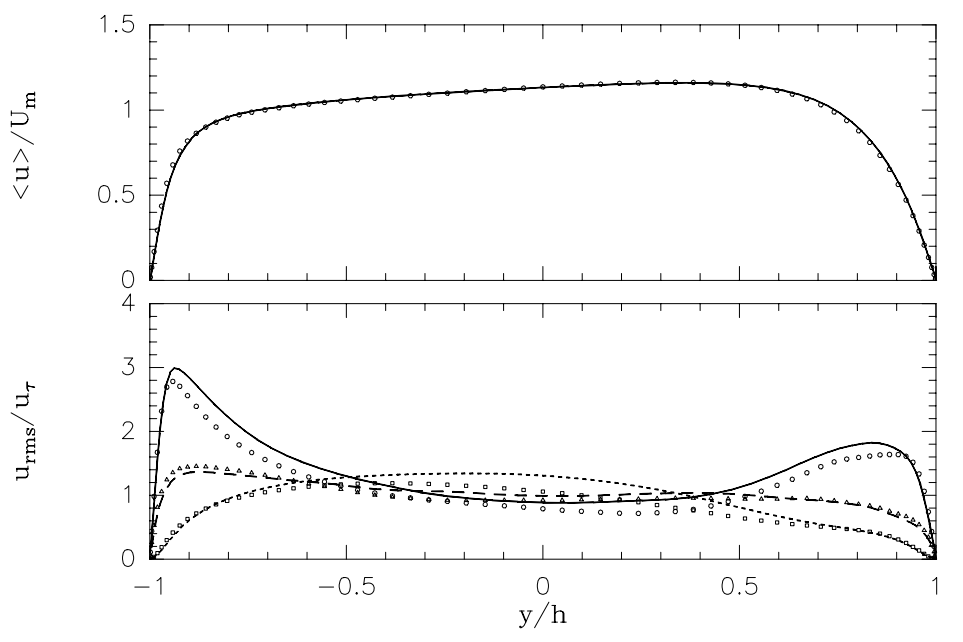

Fig. 22. Mean velocity and turbulence intensities $\left(R e=5700, R o_{\mathrm{g}}=21\right)$. 一, 。, $\sqrt{\left\langle\bar{u}^{\prime 2}\right\rangle} / u_{\tau} ; \ldots, \sqcup, \sqrt{\left\langle\bar{v}^{\prime 2}\right\rangle} / u_{\tau} ;-\cdot-, \triangle, \sqrt{\left\langle\bar{w}^{\prime 2}\right\rangle} / u_{\tau}$. Lines: spectral-dynamic LES; symbols: LES of Piomelli and Liu [96]; courtesy Lamballais.

$R o_{\mathrm{g}}=21$ and $R e=5700$. Figure 22 shows that the mean and rms velocity profiles predicted by the two models are in excellent agreement.

We next investigate rotation regimes for which anticyclonic destabilization is achieved: this corresponds to rotation rates such that $R o_{\mathrm{g}}>1$. For these rotation rates, the shear-Coriolis instability should be at hand, as in the free-shear case, at least if the Rossby number is not too high. The computations are here performed at $R o_{\mathrm{g}}=\infty, 18,6,2$. We also study Reynolds number effects by comparing spectral-dynamic model based LES at $R e=14000$ with DNS at $R e=5000$.

We here examine the three-dimensional flow structure. Figure 23 clearly shows that rotation strongly modifies the vortex organization. We observe the following trends:

- the turbulent activity is gradually reduced near the cyclonic wall as the rotation rate is increased. For $R o_{\mathrm{g}}=2$, the very flat isosurfaces indicate an almost complete flow relaminarization. This will be confirmed by the statistics;

- on the anticyclonic region, the flow presents a strong turbulence activity. We have checked in the DNS (see Lamballais et al. [61]) the existence of large-scale longitudinal roll cells similar to those already observed in the laboratory experiments of Johnston et al. [48] and 
in the numerical simulations of Kristoffersen and Andersson [55] and Piomelli and Liu [96]. The roll cells are no longer present for $R o_{\mathrm{g}}=2$;

- the vortical structures are more and more organized as rotation is increased, and their inclination with respect to the wall is reduced. This is clearly demonstrated by considering the statistics of the inclination angle of the vorticity vector (see Lamballais et al. [61]).

It is important to note that the LES are capable to reproduce all the characteristic features of the flow organization. The subgridscale model is indeed able to capture cyclonic relaminarization with inactive turbulent motions as well as detailed turbulent flow organization on the anticyclonic side (Fig. 23).
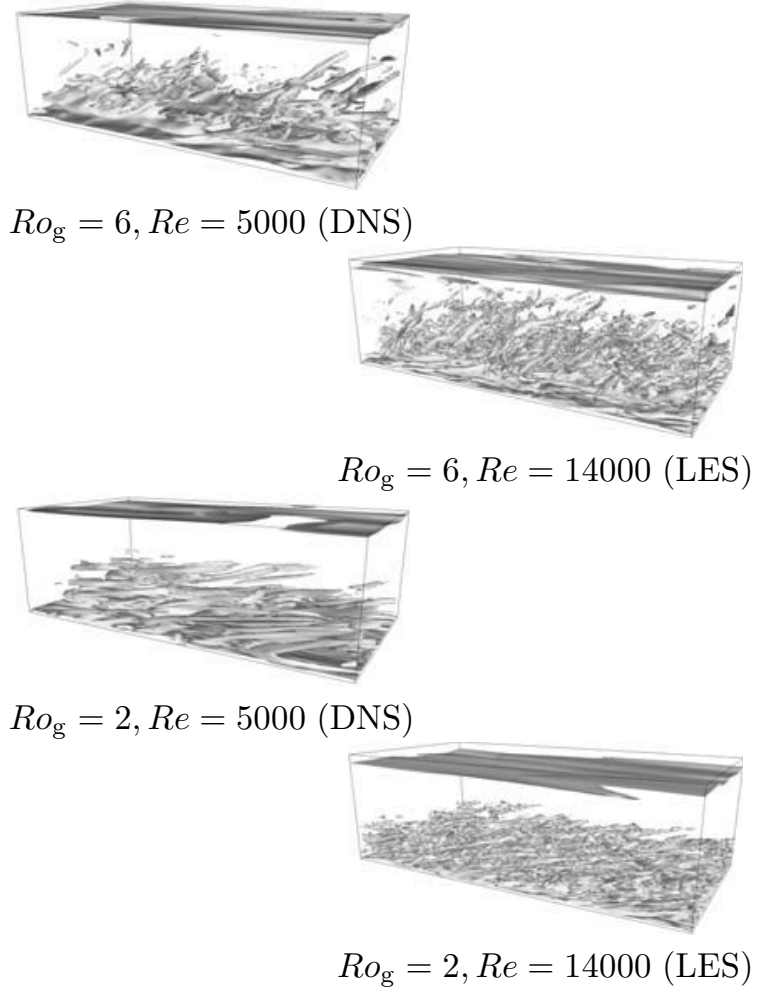

Fig. 23. Isosurfaces of vorticity modulus $\omega=3 U_{\mathrm{m}} / h$ for $R o_{\mathrm{g}}=6$ or $\omega=$ $2.25 U_{\mathrm{m}} / h$ for $R o_{\mathrm{g}}=2$ (for the DNS results, only a quarter of the computational domain is presented). 
As far as the statistical quantities are concerned, Figure 24, taken from Lamballais et al. [61] clearly shows that the important physical result corresponding to the establishment of the $R_{\mathrm{o}}(y)=-1$ plateau, predicted by the DNS, is well reproduced by the LES. Furthermore, Lamballais et al. [61] have thoroughfully checked various statistical quantities based upon the mean velocity field, the fluctuating velocity field or the fluctuating vorticity field. An excellent agreement with the DNS is obtained and the LES are able to correctly reproduce all the anisotropy characteristics of the flow. It is important to note that the rotating channel is quite a challenging test case for the one-point closure models based of a Reynolds Averaged Navier-Stokes approach and quite sophisticated models have to be designed to obtain satisfactory results. Subgrid-scale models turn out to be much more universal, since identical models can be used for different flows submitted to various external forces.
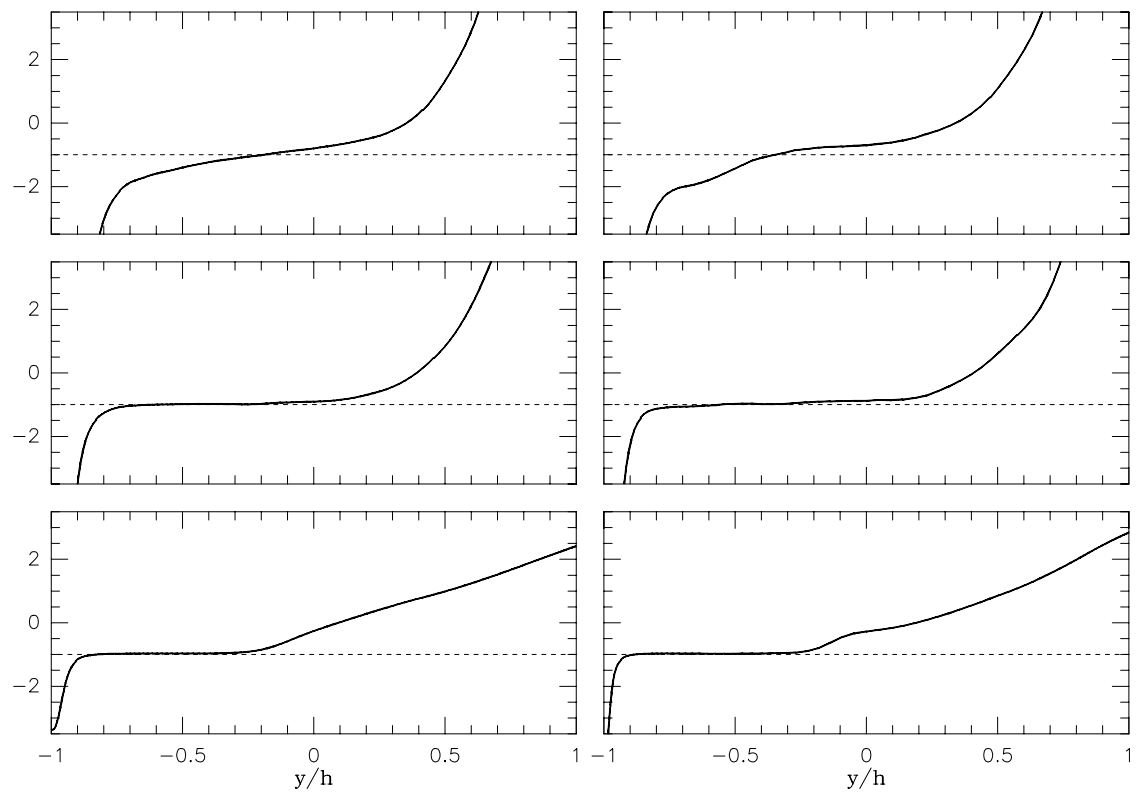

Fig. 24. Final local Rossby in the DNS (left, global Reynolds number 5000) and LES (right, Reynolds 14000) of a rotating channel. From top to bottom, initial Rossby at the wall: 18,6 and 2 . 


\subsubsection{Homogeneous turbulence}

Bartello et al. [9] have performed numerical simulations investigating the formation and stability of quasi-twodimensional coherent vortices in rotating homogeneous three-dimensional flow. Isolated coherent two-dimensional vortices obtained from a purely two-dimensional decay simulation, were superposed with a low-amplitude three-dimensional perurbation, and used to initialize a first set of simulations. In the non-rotating case, a threedimensionalization of all vortices was observed. Conversely, when $2 \Omega \approx$ $[\vec{\omega} \cdot \vec{\Omega}]_{\mathrm{rms}}$, a rapid destablization of the anticyclones was observed to occur, whereas the initial two-dimensional cyclonic vortices persisted throughout the simulation. At larger $\Omega$, both cyclones and anticyclones remained twodimensional, consistent with the Taylor-Proudman theorem. A second set of simulations starting from isotropic three-dimensional fields was initialized by allowing a random field to evolve with $\Omega=0$ to a fully-developed state. When the simulation were continued with $2 \Omega \approx[\vec{\omega} \cdot \vec{\Omega}]_{\mathrm{rms}}$, the threedimensional flow was observed to organize into two-dimensional cyclonic vortices. At large $\Omega$, two-dimensional anticyclones also emerged from the initially-isotropic flow.

\section{LES of flows of geophysical interest}

Most of the flows encountered in the atmosphere or the ocean are composed of interacting waves and turbulence. Geophysical eddies and turbulence often originate from the development of instabilities resulting from the combined effects of density gradients and rotation, and these strongly affect the dynamics over a large range of scales. We summarize here the results of DNS and LES aimed at investigating the effects of stable or unstable density stratification and/or solid-body rotation on turbulence and coherent vortices, and we particularly focus our study on three-dimensional processes.

\subsection{Baroclinic eddies}

The baroclinic instability results from the combined effects of horizontal temperature gradients and fast rotation on a stably-stratified fluid. It corresponds to a very efficient mechanism of conversion of potential energy into horizontal kinetic energy. When one considers horizontal scales of the order of the internal Rossby radius of deformation $(\approx 1000 \mathrm{~km}$ in the atmosphere and $\approx 50 \mathrm{~km}$ in the ocean, at mid-latitude), this instability becomes very active and gives rise to "baroclinic" eddies. Garnier [37], Garnier et al. [38,39] have performed direct and large-eddy simulations of baroclinic jet flows instabilities with the goal to study the 
nature of the coherent vortices and in particular the asymmetry between cyclonic and anticyclonic eddies. Garnier et al. $[38,39]$ have considered a stably-stratified medium associated with a constant vertical mean density gradient characterized by a constant Brunt-Vaissala frequency $N$. The initial basic state consits in an horizontal density front oriented in the meridional direction $\vec{y}$. The rotation vector $\vec{\Omega}$ is oriented in the $z$ direction. Let $f=2 \Omega$ be the Coriolis parameter. In the limit of fast rotation and strong stratification, it can be shown that the density front has to be associated with a basic velocity profile. Indeed, the geostrophic equilibrium corresponding to a balance between the Coriolis force and the pressure gradient and the hydrostatic balance imply that this basic state has to satisfy the thermal wind equation:

$$
\frac{\partial \vec{u}_{\mathrm{H}}}{\partial z}=-\frac{g}{\rho_{0} f} \vec{z} \times \vec{\nabla}_{\mathrm{H}} \rho,
$$

where $\vec{a}_{\mathrm{H}}$ stands for the horizontal projection of the vector $\vec{a}$ on the horizontal plane. The meridional density gradient then give rise to a mean velocity corresponding to a jet, sheared along the vertical direction and directed to the east at the top of the domain and to the west at the bottom. When a small random perturbation is superposed to this basic state the flow becomes unstable. The nature of the instability is however very different depending upon the characteristic parameters. The two non-dimensionalized parameters are the Rossby $(R o)$ and Froude $(F r)$ numbers (see Garnier et al. $[38,39])$.

First, Garnier et al. [38] have used direct numerical simulations to carry out linear stability studies. They have shown the existence of a critical value of the ratio $R o / F r=1.5$ constituting the threshold between to distinct regimes:

1) $\mathrm{Ro} / \mathrm{Fr}>1.5$ : the instability is weak and mainly barotropic: it is of Kelvin-Helmholtz type and is associated with the inflexional nature of the mean velocity profile;

2) $R o / F r \leq 1.5$ : the baroclinic instability corresponding to a conversion of potential energy associated with the horizontal density gradient into horizontal kinetic energy can develop. The amplification of the perturbations is much stronger than in the barotropic case.

We now concentrate on the second regime $R o / F r \leq 1.5$ : here $R o / F r=0.5$. The Rossby number is fixed to 0.1 . The numerical code is similar to the channel flow study previously described except that compact differences schemes are here used into two spatial directions. The Reynolds number is low in the DNS $(R e=400)$ and much higher in the LES $(R e=10000)$. 


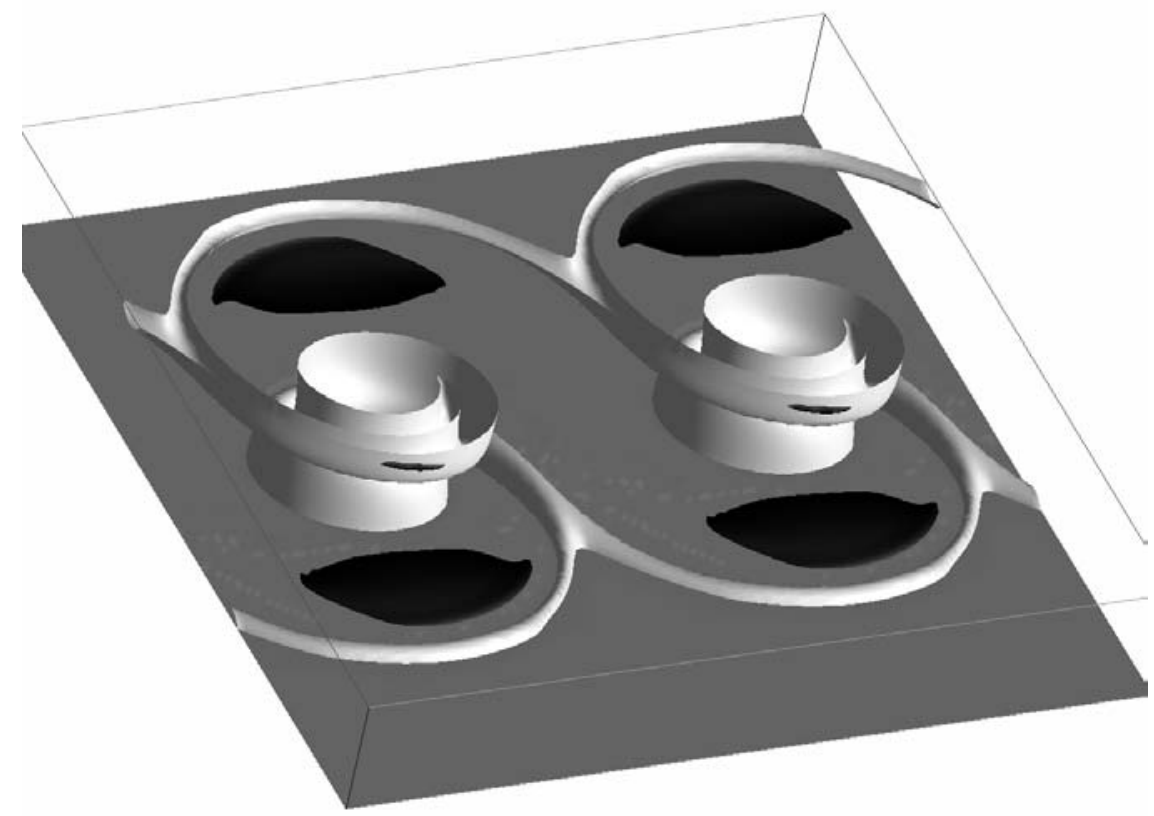

Fig. 25. Iso-surfaces of vertical vorticity; $n$ light-gray: cyclonic vorticity, black: anticyclonic vorticity (courtesy Garnier).

\subsubsection{Synoptic-scale instability}

Figure 25 shows the vorticity structure obtained by DNS once the instability has fully developed. We observe the formation of cyclonic eddies of strong intensity, composed of nearly two-dimensional cores between which braids of very high cyclonic vorticity are formed. The vorticity maxima are observed within these braids and correspond here to $\approx 8$ times the vorticity maximum of the initial mean velocity profile. The vorticity intensification in the anticyclonic eddies is weaker (3 times the initial vorticity): we have checked that those are far more three-dimensional than the cyclonic eddies and strongy stretched by them. The asymmetry cyclones/anticyclones is clear and the vorticity maxima are cyclonic and are localized in very concentrated regions of the space. Contrarily, the anticyclonic vorticity is much more spread. 


\subsubsection{Secondary cyclogenesis}

In the previous DNS, the late stage of the cyclogenesis was dominated by dissipative and diffusive effects. We have thus performed high Reynolds number LES at $R e=10000$ to study the flow development subsequent to the occlusion process. The subgrid-scale structure function model with a cusp given by equation (7.3) have been used. Here the grid is nonisotropic $\Delta x \neq \Delta y \neq \Delta z$ and the formulation for non-uniform gird, given by equations (5.7) and (5.8), is used.

Figure 26 shows a time evolution of the vorticity contours of a cyclonic eddies. As compared with the DNS presented in the preceeding section, one may notice that the spiralling of the vorticity contours inside the core of the cyclonic eddies is much more pronounced. Due to viscous effects, the vorticity was indeed homogenized in the DNS. We have checked that the frontal region are much steeper in the LES indicating more energy near the wavenumber cut-off. The steepening of the fronts is associated with the appearance of a secondary instability resulting in a local intensification of the vertical vorticity. This instability seems to take place in regions where the local values of the Rossby and Froude number $\operatorname{Ro}(\vec{x})$ and $\operatorname{Fr}(\vec{x})$ verify the criterion $\operatorname{Ro}(\vec{x}) / \operatorname{Fr}(\vec{x}) \leq 1.5$. The potential energy associated with local horizontal fronts is then converted into horizontal kinetic energy and gives rise to vertical vorticity intensification. It is important to notice that if the structure model without cusp is used excessive accumulation of energy is observed at the smallest scales eventually leading to numerical divergence. This demonstrates the importance of the cusp-like behaviour and the feasibility of the subgrid-scales previously described for the LES of geophysical flows with quasi-twodimensional regions and sharp frontal regions.

Note that the present results have been compared by Lesieur et al. [70] with satellite observations corresponding to the severe storm of 26 Dec. 1999. This storm, together with its companion on 28 December, caused casualties and immense damage in France and neighbouring countries. Lesieur et al. [70] discuss possible analogies and differences, as well as some consequences in terms of numerical weather forecasting.

Note that the same subgrid-scale models and the same numerical code has also been successfully used to study the combined effects of unstable density stratification and rotation. Padilla-Barbosa and Métais [91] have indeed performed LES of rotating turbulent convection with an application to oceanic deep-water formation. 

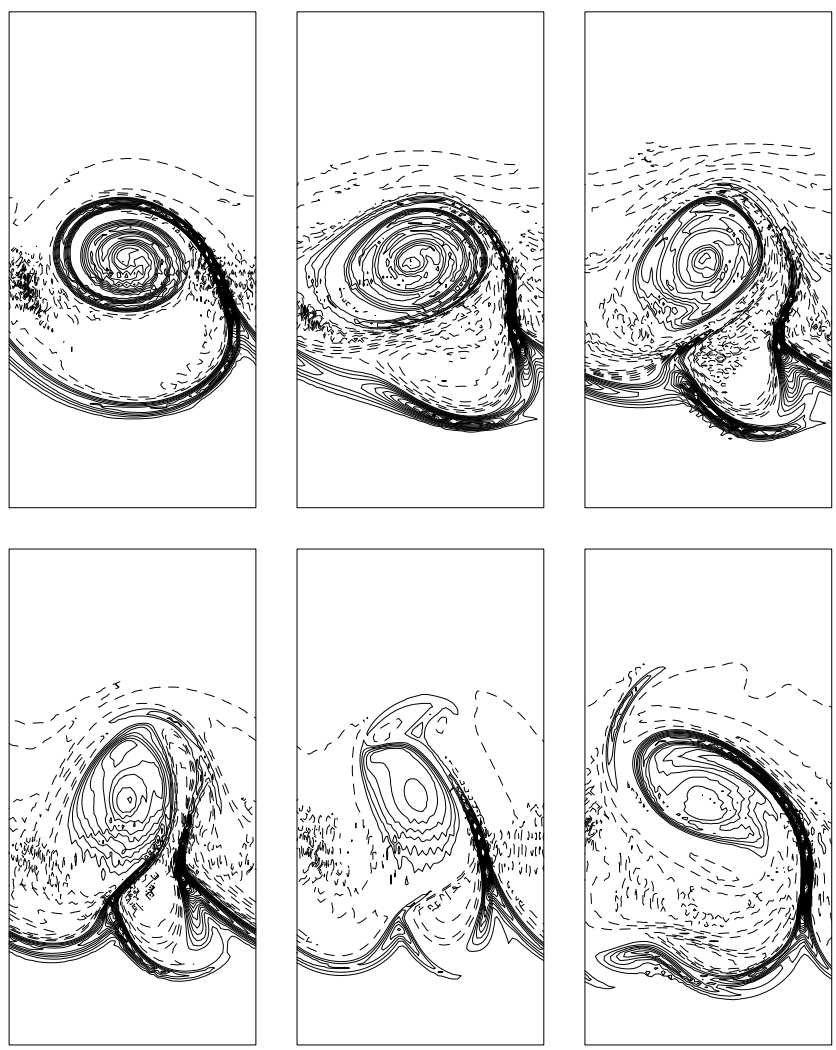

Fig. 26. LES simulation at $R e=10000$ : time evolution of the vertical vorticity isocontours at the top of the computational domain. Solid-lines cyclonic vorticity; dashed lines anticyclonic vorticity.

\section{LES of compressible turbulence}

Compressible turbulence has extremely important applications in subsonic, supersonic and hypersonic aerodynamics. More generally, and even at low Mach numbers, strong density differences due to an intense heating (in combustion for instance) may have profound consequences on the flow structure and the associated mixing. Heating a wall may, for instance, completely destabilize a boundary layer, due to the generalized inflexion-point instability. Examples and details are given below. 


\subsection{Compressible LES equations}

We here briefly recall the specific difficulties attached to the filtered compressible Navier-Stokes equations. Details may be found in Lesieur and Comte [68] and Métais et al. [81]. The momentum equation with variable density, $\rho(\vec{x}, t)$, and for a newtonian fluid can be written in the so-called conservative form as:

$$
\frac{\partial}{\partial t}\left(\rho u_{i}\right)+\frac{\partial}{\partial x_{j}}\left(\rho u_{j} u_{i}\right)=-\frac{\partial}{\partial x_{j}}\left[p \delta_{i j}+\frac{2 \mu}{3}(\vec{\nabla} \cdot \vec{u}) \delta_{i j}-2 \mu D_{i j}\right] .
$$

Similarly, the continuity equation can be expressed as:

$$
\frac{\partial \rho}{\partial t}=-\frac{\partial}{\partial x_{j}}\left(\rho u_{j}\right)
$$

These equation have to be completed by an equation representing the evolution of the total energy defined by, for an ideal gas,

$$
\rho e=\rho C_{\mathrm{v}} T+\frac{1}{2} \rho\left(u_{1}^{2}+u_{2}^{2}+u_{3}^{2}\right)
$$

as well as the equation of state.

The application of the filter operator - to these equations yields quantities of the following form:

$$
\overline{\rho \Phi}
$$

where $\Phi(\vec{x}, t)=u_{i}(\vec{x}, t), e(\vec{x}, t), T(\vec{x}, t)$, etc. These are not easily expressable as a simple function of $\bar{\rho}$ and $\bar{\Phi}$. To overcome this difficulty it is customary (see Favre [35]) to introduce the density-weighted Favre average (here density-weighted filter) denoted as ${ }^{\sim}$ :

$$
\widetilde{\Phi}=\frac{\overline{\rho \Phi}}{\bar{\rho}} .
$$

One then tries to write a closed system of equations for the variables $\widetilde{\Phi}$ and for $\bar{\rho}$. For instance, the equation for $\bar{\rho}$ is obtained through the filtered continuity equation:

$$
\begin{aligned}
& \frac{\partial \bar{\rho}}{\partial t}=-\frac{\partial}{\partial x_{j}}\left(\overline{\rho u_{j}}\right) \\
& \frac{\partial \bar{\rho}}{\partial t}=-\frac{\partial}{\partial x_{j}}\left(\bar{\rho} \tilde{u}_{j}\right) .
\end{aligned}
$$

The application of the filter - to the momentum equation gives rise to the subgrid-scale stress tensor, whose form, in the compressible case, is:

$$
T_{i j}=\bar{\rho} \tilde{u}_{i} \tilde{u}_{j}-\overline{\rho u_{i} u_{j}} .
$$


The filtered momentum equation may be closed through a classical turbulent viscosity assumption. The closure of the energy equation may be resolved by introducing an eddy-conductivity $k_{\mathrm{t}}(\vec{x}, t)$ :

$$
k_{\mathrm{t}}(\vec{x}, t)=C_{\mathrm{p}} \frac{\nu_{\mathrm{t}}(\vec{x}, t)}{P r_{\mathrm{t}}}
$$

where $\nu_{\mathrm{t}}(\vec{x}, t)$ is the turbulent viscosity. Note that the difficulties associated with the trace of the subgrid-scale tensor $T_{l l}$ may be nicely overcome by the introduction of a "macro-pressure"

$$
\varpi=\bar{p}-\frac{1}{3} T_{l l}
$$

and of a "macro-temperature"

$$
\vartheta=\widetilde{T}-\frac{1}{2 C_{\mathrm{v}} \bar{\rho}} T_{l l}
$$

as proposed by Lesieur and Comte [68]. Except for these differences, the formulation of the subgrid-scale model is identical to the incompressible case but $\nu_{\mathrm{t}}(\vec{x}, t)$ is now determined from the density-weighted filtered velocity field $\widetilde{u}_{i}$. Another important difference with the incompressible case is that the dynamic viscosity varies with temperature through the classical Sutherland empirical law.

\subsection{Heated flows}

The compressible results presented here are based upon the COMPRESS code developed in Grenoble. The details on the numerical procedure can be found in [22]. The numerical code uses curvilinear co-ordinates. The system is solved in the transformed grid by means of a extension of the fully-explicit McCormack scheme, second order in time and fourth in space, devised by Gottlieb and Turkel [44]. High-Mach number boundary layer simulations have been performed by Normand et al. $[87,88]$ which constitute a validation of the numerics and SGS model. It showed in particular the ability of the code to reproduce the effect of strong heating on Reynolds stresses. It thus can be considered as a suitable tool for prediction of heat fluxes in situations for which experimental data are absent or sparse. Indeed, the understanding of the dynamics of turbulent flows submitted to strong temperature gradients is still an open challenge for numerical and experimental research. It is of vital importance due to the numerous industrial applications such as the heat exchangers, the cooling of turbine blades, the cooling of rocket engines, etc. 


\subsubsection{The heated duct}

We want here to show the ability for LES to adequately reproduce the effects of an asymetric heat flux in a square duct flow. The details of the computations are reported in [99] and [100]. We solve the three-dimensional compressible Navier-Stokes equations with the COMPRESS code previously mentioned. The subgrid-scale model is the selective structure function model. We have successively considered the isothermal duct, at a Reynolds number $R e_{\mathrm{b}}=6000$ (based on the bulk velocity), with the four wall at the same temperature and the heated duct for which the temperature of one of the walls is imposed to be higher than the temperature of the three other walls $\left(R e_{\mathrm{b}}=6000\right)$. It is important to note that moderate resolutions are used: the grid consists of $32 \times 50 \times 50$ nodes in the isothermal case and of $64 \times 50 \times 50$ nodes in the heated case along $x$ (streamwise), $y$ and $z$ (transverse) directions. This moderate resolution renders the computation very economical compared with a DNS. One crucial issue in LES is to have a fine description of the boundary layers. In order to correctly simulate the near-wall regions, a nonuniform (orthogonal) grid with a hyperbolic-tangent stretching is used in the $y$ and $z$ directions: the minimal spacing near the walls is here 1.8 wall units. The Mach number is $M=0.5$ based upon the bulk velocity and the wall temperature.

We have first validated our numerical procedure by comparing our results, for the isothermal duct, with previous incompressible DNS results [40]: a very good agreement was obtained at a drastically reduced computer cost. The flow inside a duct of square cross section is characterized by the existence of secondary flows (Prandtl's flow of second kind) which are driven by the turbulent motion. The secondary flow is a mean flow perpendicular to the main flow direction. It is relatively weak $(2-3 \%$ of the mean streamwise velocity), but its effect on the transport of heat and momentum is quite significant. If a statistical modelling approach is employed, elaborate second-order models have to been utilized to be able to accurately reproduce this weak secondary flow. Figure 27 a shows the contours of the streamwise vorticity in a quarter of a cross section. The secondary flow vectors reveal the existence of two streamwise counter-rotating vortices in each corner of the duct. The velocity maximum associated with this flow is $1.169 \%$ of the bulk velocity: this agrees very well with experimental measurements. It shows the ability for LES to accuratly reproduced statistical quantities. Figure 27b shows the instantaneous flow field for the entire duct cross-section. As compared Figure 27a, it clearly indicates a very pronounced flow variability with an instantaneous field very distinct from the mean field. The maximum for the transverse fluctuating velocity field is of the order of ten times the maximum for the corresponding mean velocity field. As far as the vorticity is concerned, the transverse motions are 

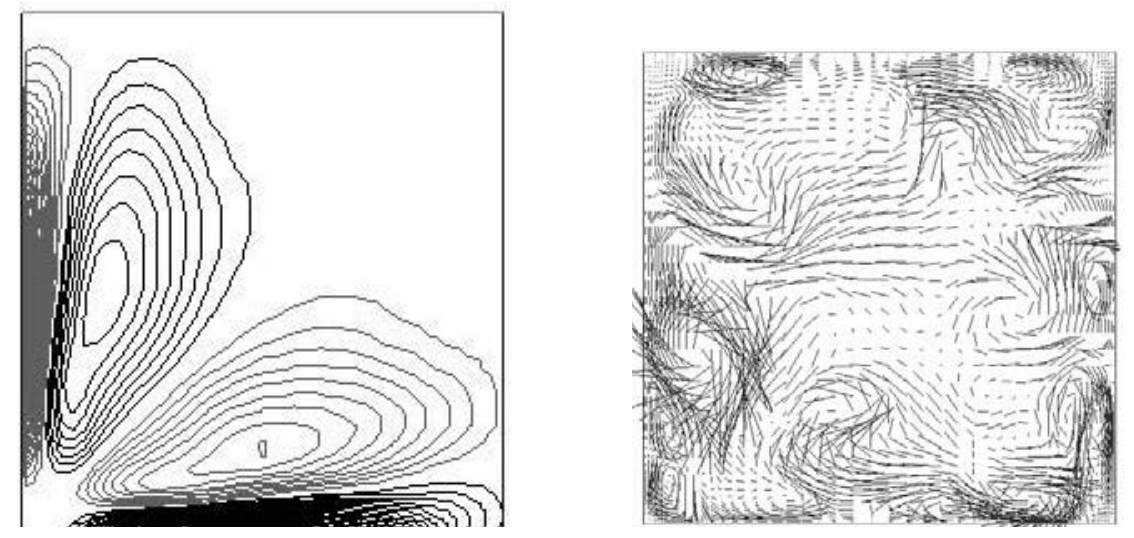

a)

b)

Fig. 27. a) Ensemble averaged streamwise vorticity contours; b) vectors of the instantaneous velocity field (courtesy Salinas-Vasquez).

associated with streamwise vorticity generation, whose maximum is about one third of the transverse vorticity maximum.

In the heated case, Salinas and Métais [100] have investigated the effect of the heating intensity by varying the temperature ratio between the hot wall and the other walls. When the heating is increased, an amplification of the mechanism of ejection of hot fluid from the heated wall is observed. Figure 28 shows temperature structures near the heated wall of the duct. Only one portion of the duct is here represented. As shown in Figure 28, these ejections are concentrated near the middle plane of the heated wall. This yields a strong intensification of the secondary flow. It is also shown that the turbulent intensity is reduced near the heated wall with strong heating due to an increase of the viscous effect in that region.

\subsubsection{Towards complex flow geometries}

Several applications of LES to compressible flows in geometries of inductrial interest are presented in Lesieur and Comte [68] and Métais et al. [81]. We here briefly mentioned two of these applications.

The first application is a LES of the detached boundary layer over a curved compression ramp at Mach 2.5 modelling the wind-side region of the body-flap of HERMES during its projected re-entry. Note that the simulated Mach number is lower than for real situations. Indeed, the external Mach number relevant to the shuttle is about 10 (altitude $50 \mathrm{~km}$, incidence $30^{\circ}$, flap extension angle $\alpha_{0}=20^{\circ}$ ). The whole computational domain is 


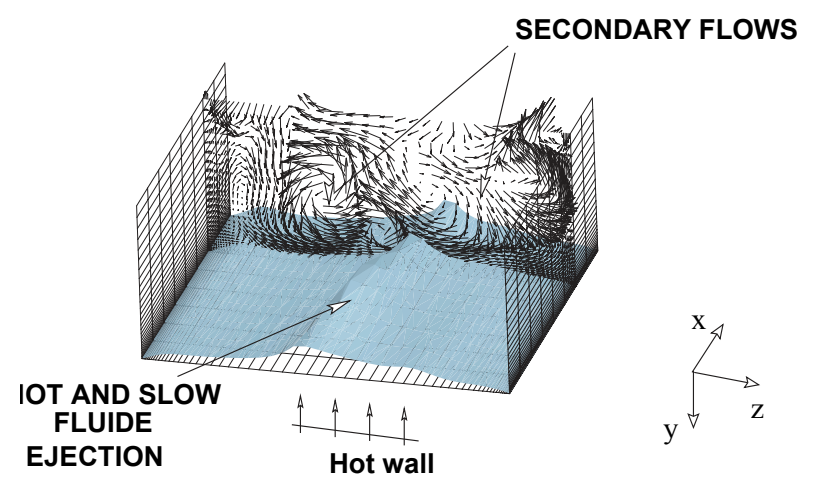

Fig. 28. Large scale motion over the hot wall in a heated duct $\left(T_{\mathrm{h}} / T_{\mathrm{w}}=2.5\right)$. Instantaneous transversal vector field and a isosurface of temperature $\left(T / T_{\mathrm{w}}=\right.$ 2.1) (courtesy Salinas-Vasquez).

contained within the bow shock. The grid used is shown, upside down, in Figure 29. The simulation is therefore performed at the maximal Reynolds number permitted by our resolution, that is $R e_{\delta_{i}}=280$, where $\delta_{i}$ is the upstream displacement thickness of the boundary layer. The first part of the boundary (up to $13.6 \mathrm{~m}$ away from the nose) is curved. It corresponds to the wind side of the body. The ramp corresponds to the body flap, assumed to be flat. For computational reasons, it is prolonged by a fictitious horizontal surface introducing a cut-off with the lee-side of the flap and the after-body. This enables the prescription of well-posed boundary conditions at the exit of the domain. The wall temperature is $T_{\mathrm{w}}=290 \mathrm{~K}$ and the "external" (outside of the boundary layer, but inside the bow shock) temperature is $T_{\infty}=460 \mathrm{~K}$. The adiabatic recovery temperature, defined by

$$
T_{\mathrm{ad}}=T_{\infty}\left(1+\sqrt{\operatorname{Pr}} \frac{\gamma-1}{2} M_{\infty}^{2}\right)
$$

is $T_{\text {ad }}=1047 \mathrm{~K}$, yielding $T_{\mathrm{w}} / T_{\mathrm{ad}}=0.277$. The ramp is therefore very cool with respect to the fluid, which models the radiative balance of the true shuttle during its re-entry.

Experimental evidence of (streamwise counter-rotating) Görtler vortices in a similar case was brought in particular by [101], but the consequence of these vortices on the wall heat flux has remained an open question. Figure 30 shows such Görtler vortices, obtained from a 3D simulation performed with the selective structure-function model in a domain of spanwise extension equal to $4.5 \delta_{i}$. One clearly sees two large structures, cross-cuts of which show that each of them corresponds to a pair of counter-rotating Görtler 


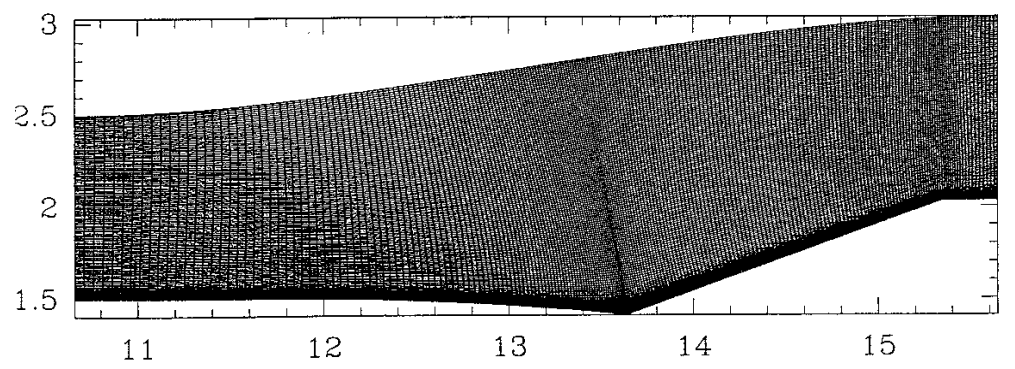

Fig. 29. Transverse section of the $220 \times 140 \times 25$ - point grid used for the simulation of the transition on a the curved ramp (angle $20^{\circ}$ ). The axes are graded in metres, counted from the nose of the full-size shuttle. The spanwise size of the domain is 4.5 the displacement thickness $\delta_{i}$ of the boundary layer prescribed at the upstream boundary.

vortices. We have checked that the extreme values of the temperature fluctuations close to the wall associated with these structures are $\pm 90 \mathrm{~K}$. These $30 \%$ of temperature fluctuations induce huge fluctuations of the Stanton number (normalized heat-flux), between $2 \times 10^{-3}$ and $14 \times 10^{-3}$, with an average of about $6 \times 10^{-3}$. The rms of the Stanton-number fluctuations is thus $133 \%$. The same trend is observed for the skin-friction coefficient $C_{\mathrm{f}}$. Note that LES is the only available tool able to reproduce such a strong variability for high-Reynolds number flows. Time-averaged plots also prove that the Görtler vortices are, in this simulation, fairly stable in time. This is likely to enhance considerably their destructive effects on the material of the body flap.

The second example is taken from the recent work by Dubief and Delcayre [30]. It consists in the LES of a transonic flow past a rectangular cavity. It required the implementation of domain decomposition in the COMPRESS code. The Reynolds number is here $1.25 \times 10^{6}$ based upon the external velocity and the depth of the cavity and the Mach number is 0.95. The Figure 31 displays, through the $Q$ criterion, the vortices which are shed by the cavity. The main interest of this flow is related with aeroacoustical aspects and with the noise generated by the various eddies. It has been checked that the present LES is able to correctly reproduce the characteristics frequencies which are experimentally measured. This makes the LES a precious tool for aeroacoustics studies. 


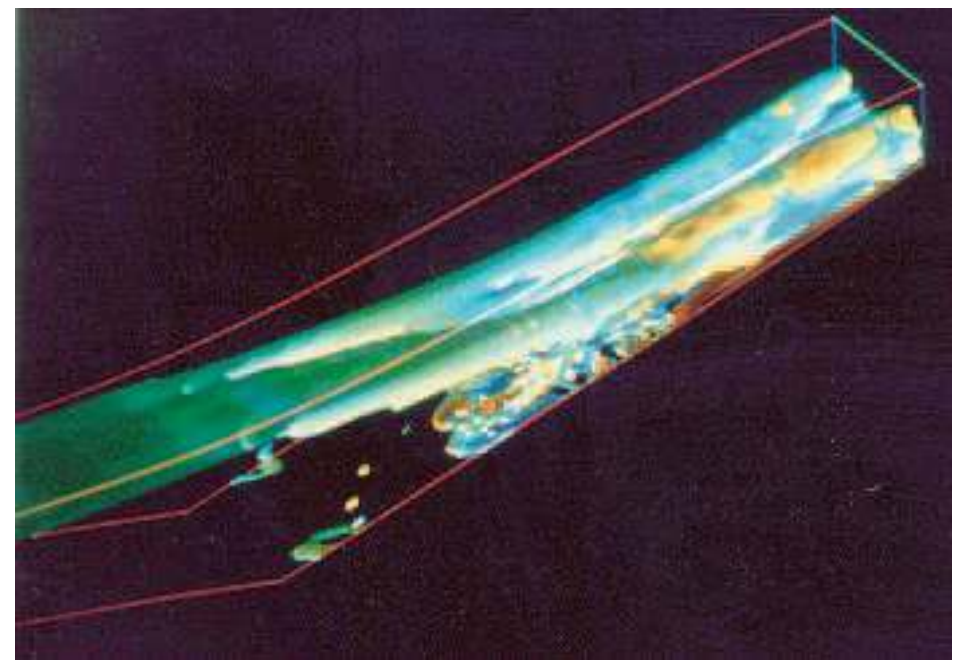

Fig. 30. Ramp flow. Zoom on the hinge and body-flap region showing an isosurface of the vorticity magnitude. This surface is coloured by temperature. This shows clearly that hot fluid in the outer part of the boundary layer is being downwashed to the wall, which brings about wall-heat-flux fluctuations.

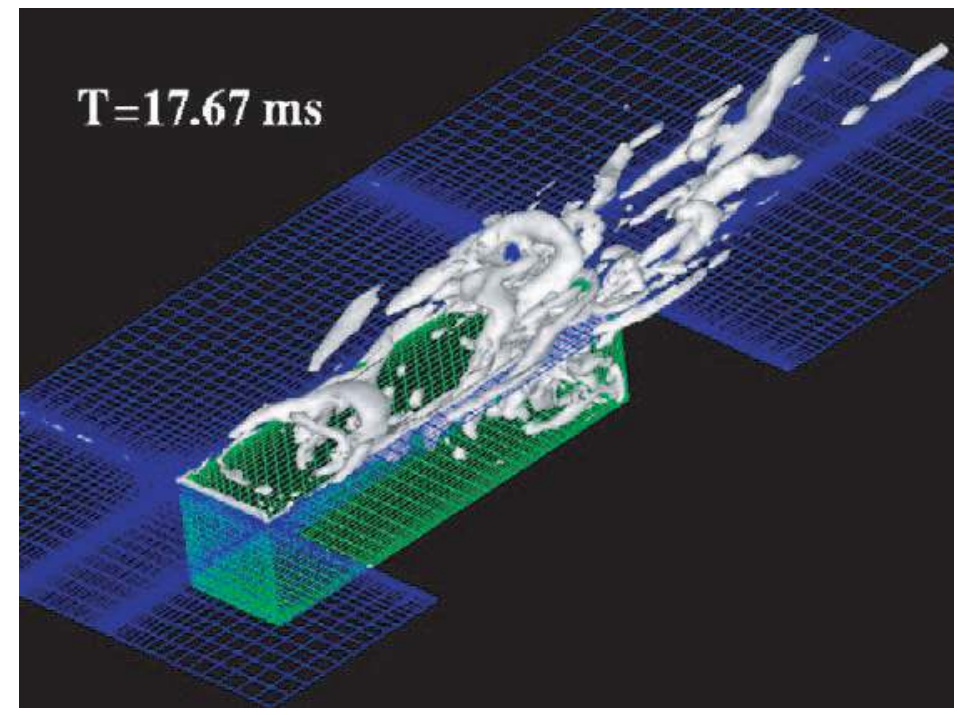

Fig. 31. LES of a transonic flow past a rectangular cavity at $R e=1.25 \times 10^{6}$ and Mach $=0.95$. White: vortices identified through the $Q$-criterion. 


\section{Conclusion}

Turbulence plays a major role in the aerodynamics of cars, trains and planes, combustion in engines, acoustics, cooling of nuclear reactors, dispersion of pollution in the atmosphere and the oceans, or magnetic-field generation in planets and stars. Applications of turbulence, industrial in particular, are thus immense. Since the development of computers in the sixties, socalled industrial numerical models have been created. These models solve Reynolds ensemble-averaged equations of motions (RANS), and they require numerous empirical closure hypotheses which need to be adjusted on given particular experimentally-documented cases. RANS are widely used in the industry. However, it has become clear than RANS models suffer from a lack of universality and require specific adjustments when dealing with a flow submitted to such effects as separation, rotation, curvature, compressibility, or strong heat release.

Classical turbulence modelling, based on one-point closures and a statistical approach allow computation of mean quantities. In many cases, it is however necessary to have access to the fluctuating part of the turbulent fields such as the pollutant concentration or temperature: LES is then compulsory. Large-eddy simulations (LES) of turbulent flows are extremely powerful techniques consisting in the elimination of small scales by a proper low-pass filtering, and the formulation of evolution equations for the large scales. The latter have still an intense spatio-temporal variability. History of large-eddy simulations (LES) started also at the beginning of the sixties with the introduction of the famous Smagorinsky's [105] eddy viscosity. Due to the tremendous progress in scientific computing and in particular of parallel computing, LES, which were first confined to very simple flow configurations, are able to deal with more and more complex flows. We have here shown several examples of applications showing that LES are an invaluable tool to decipher the vortical structure of turbulence. Together with DNS, LES is then able to perform deterministic predictions (of flows containing coherent vortices, for instance) and to provide statistical information. The last is very important for assessing and improving one-point closure models, in particular for turbulent flows submitted to external forces (stratification, rotation, ...) or compressibility effects. The ability to deterministically capture the formation and ulterior evolution of coherent vortices and structures is very important for the fundamental understanding of turbulence and for designing efficient turbulent flow control.

The complexity of problems tackled by LES is continuously increasing, and this has nowadays a decisive impact on industrial modelling and flow control. Among the current challenges for LES in dealing with very complex geometries (like the flow around an entire car) are the development of efficient wall functions, the use of unstructured meshes and the use of 
adaptative meshes. However, the design of efficient industrial turbulence models will necessarily require an efficient coupling of LES and RANS techniques.

The results presented have greatly benefitted from the contributions of E. Briand, P. Comte, E. David, F. Delcayre, Y. Dubief, E. Garnier, E. Lamballais, M. Lesieur, M. Salinas-Vasquez, C. Silva, G. Silvestrini, G. Urbin. We are indebted to P. Begou for the computational support. Some of the computations were carried out at the IDRIS (Institut du Développement et des Ressources en Informatique Scientifique, Paris).

\section{References}

[1] A. Abbà, C. Cercignani, L. Valdettaro and P. Zanini, LES of turbulent thermal convection, in Direct and Large Eddy Simulation II, edited by J.P. Chollet, P.R. Voke and L. Kleiser (Kluwer Academic Publishers, 1997) pp. 147-156.

[2] K. Akselvoll and P. Moin, Large Eddy Simulation of turbulent confined coannular jets and turbulent flow over a backward facing step, TF 63 (Stanford University, 1995).

[3] J.C. André and M. Lesieur, J. Fluid Mech. 81 (1977) 187-207.

[4] R.A. Antonia, M. Teitel, J. Kim and L.W.B. Browne, J. Fluid Mech. 236 (1992) 579-605.

[5] C. Airiau, Stabilité linéaire et faiblement non-linéaire d'une couche limite laminaire incompressible par un système d'équations parabolisé (PSE), Ph.D. Thesis (Toulouse University, 1994).

[6] M. Arnal and R. Friedrich, Large-eddy simulation of a turbulent flow with separation, in Turbulent Shear Flows 8, edited by F. Durst, R. Friedrich, B.E. Launder et al. (Springer, 1993) p. 169.

[7] G. Barenblatt and V.M. Prostokishin, J. Fluid Mech. 248 (1993) 513-529.

[8] J. Bardina, J.H. Ferziger and W.C. Reynolds, Improved subgrid model for largeeddy simulation, AIAA paper Nos. 80-1357 (1980).

[9] P. Bartello, O. Métais and M. Lesieur, J. Fluid Mech. 273 (1994) 1-29.

[10] C. Basdevant and R. Sadourny, J. Mec. Theor. Appl., Numéro Spécial (1983) 243269.

[11] L.P. Bernal and A. Roshko, J. Fluid Mech. 170 (1986) 499-525.

[12] P. Bertolotti and T. Herbert, Theoret. and Comp. Fluids Dynamics 3 (1991) 117124.

[13] M. Brachet, C.R. Acad. Sci. Paris II 311 (1990) 775-780.

[14] E. Briand, Dynamique des structures cohérentes en couche limite transitionnelle et turbulente étudiée par simulation des grandes échelles, Ph.D. Thesis (National Polytechnic Institute, Grenoble, 1999).

[15] F.K. Browand and T.R. Troutt, J. Fluid Mech. 93 (1980) 325-336.

[16] G. Broze and F. Hussain, J. Fluid Mech. 311 (1996) 37-71.

[17] S. Cerutti, C. Meneveau and O.M. Knio, J. Fluid Mech. (2000) (in press).

[18] J.P. Chollet, Turbulence tridimensionnelle isotrope : modélisation statistique des petites échelles et simulations numérique des grandes échelles, Thèse de Doctorat d'État (Grenoble, 1984).

[19] J.P. Chollet and M. Lesieur, J. Atmos. Sci. 38 (1981) 2747-2757. 
[20] J.P. Chollet and M. Lesieur, Modélisation sous maille des flux de quantité de mouvement et de chaleur en turbulence tridimensionnelle isotrope, La Météorologie, 29-30 (1982) pp. 183-191.

[21] R.A. Clark, J.H. Ferziger and W.C. Reynolds, J. Fluid Mech. 91 (1979) 1-16.

[22] P. Comte, Numerical methods for compressible flows, in Computational Fluid Dynamics, Les Houches Session LIX, 1993, edited by M. Lesieur, P. Comte and J. Zinn-Justin (Elsevier Science, 1996).

[23] P. Comte, Y. Fouillet and M. Lesieur, Simulation numérique des zones de mélange compressibles. Revue scientifique et technique de la defense, $3^{\mathrm{e}}$ trimestre (1992) pp. $43-63$.

[24] P. Comte, M. Lesieur and E. Lamballais, Phys. Fluids A 4 (1992) 2761-2778.

[25] P. Comte, J.H. Silvestrini and P. Bégou, Eur. J. Mech. B/Fluids 17 (1998) 615-637.

[26] J. Cousteix, Turbulence et couche limite, CEPADUES (1989).

[27] E. David, Modélisation des Écoulements Compressibles et Hypersoniques : une Approche Instationnaire, Ph.D. Thesis (National Polytechnic Institute, Grenoble, 1993).

[28] F. Delcayre, Étude par simulation des grandes échelles d'un écoulement décollé : la marche descendante, Ph.D. Thesis (National Polytechnic Institute, Grenoble, 1999).

[29] J.A. Domaradzki, R.W. Metcalfe, R.S. Rogallo and J.J. Riley, Phys. Rev. Lett. 58 (1987) 547-550.

[30] Y. Dubief and F. Delcayre, J. Turbulence 1 (2000) 011.

[31] F. Ducros, Simulation numérique directe et des grandes échelles de couches limites compressibles, Ph.D. Thesis (National Polytechnic Institute, Grenoble, 1995).

[32] F. Ducros, P. Comte and M. Lesieur, Direct and large-eddy simulations of a supersonic boundary layer, in Selected Proceedings of Turbulent Shear Flows 9 (Springer, 1995) pp. 283-300.

[33] F. Ducros, P. Comte and M. Lesieur, J. Fluid Mech. 326 (1996) 1-36.

[34] N.M. El-Hady and T.A. Zang, Theoret. Comput. Fluid Dynamics 7 (1995) 217-240.

[35] A. Favre A., J. de Mécanique 4 (1965) 361.

[36] Y. Fouillet, Contribution à l'étude par expérimentation numérique des écoulements cisaillés libres. Effets de compressibilité, Ph.D. Thesis (National Polytechnic Institute, Grenoble, 1992).

[37] E. Garnier, Étude numérique des instabilités de jets beroclines, Ph.D. Thesis (Natl. Polytech. Inst., Grenoble, 1996).

[38] E. Garnier, O. Métais and M. Lesieur, J. Atmos. Sci. 55 (1998) 1316-1335.

[39] E. Garnier, O. Métais and M. Lesieur, C.R. Acad. Sci. Paris Sér. II B 323 (1996) 161-168.

[40] S. Gavrilakis, J. Fluid Mech. 244 (1986) 101.

[41] M. Germano, J. Fluid Mech. 238 (1992) 325-336.

[42] M. Germano, U. Piomelli, P. Moin and W. Cabot, Phys. Fluids A 3 (1991) 17601765.

[43] M.A. Gonze, Simulation Numérique des Sillages en Transition à la Turbulence, Ph.D. Thesis (National Polytechnic Institute, Grenoble, 1993).

[44] D. Gottlieb and E. Turkel, Math. Comp. 30 (1976) 703.

[45] T. Herbert, Ann. Rev. Fluid Mech. 20 (1988) 487-526.

[46] J.C.R. Hunt, A.A. Wray and P. Moin, Eddies, stream, and convergence zones in turbulent flows. Center for Turbulence Research Rep., CTR-S88 (1988) p. 193. 
[47] J. Jimenez and A.A. Wray, J. Fluid Mech. 373 (1998) 255-285.

[48] J.P. Johnston, R.M. Halleen and D.K. Lezius, J. Fluid Mech. 56 (1972) 533-557.

[49] S. Jovic and M. Driver, Backward-facing step measurement at low Reynolds number $R e_{\mathrm{h}}=5000$, Ames Research Center, NASA Technical Memorandum, 108807 (1994).

[50] J. Kim, The effect of rotation on turbulence structure, in Proc. 4th Symp. on Turbulent Shear Flows, Karlsruhe, (1983) pp. 6.14-6.19.

[51] P.S. Klebanoff, K.D. Tidstrom and L.M. Sargent, J. Fluid Mech. 12 (1962) 1-34.

[52] S.J. Kline, W.C. Reynolds, F.A. Schraub and P.W. Runstadler, J. Fluid Mech. 30 (1967) 741-773.

[53] A.N. Kolmogorov, Dokl. Akad. Nauk. SSSR 30 (1941) 301-305.

[54] R.H. Kraichnan, J. Atmos. Sci. 33 (1976) 1521-1536.

[55] R. Kristoffersen and H.I. Andersson, J. Fluid Mech. 256 (1993) 163-197.

[56] A. Kuroda, Direct-numerical simulation of Couette-Poiseuille flows, Ph.D. Thesis (University of Tokyo, 1990).

[57] E. Lamballais, Simulations numériques de la turbulence dans un canal plan tournant, Ph.D. Thesis (National Polytechnic Institute, Grenoble, 1996).

[58] E. Lamballais, M. Lesieur and O. Métais, Int. J. Heat and Fluid Flow 17 (1996) 324-332.

[59] E. Lamballais, M. Lesieur and O. Métais, C. R. Acad. Sci. Sér. II B 323 (1996) 95-101.

[60] E. Lamballais, O. Métais and M. Lesieur, Influence of a spanwise rotation upon the coherent-structure dynamics in a turbulent channel flow, in Direct and Large Eddy Simulation II, edited by J.P. Chollet, P.R. Voke and L. Kleiser (Kluwer Academic Publishers, 1996) pp. 225-236.

[61] E. Lamballais, O. Métais and M. Lesieur, Theoret. Comput. Fluid Dynamics 12 (1998) 149-177.

[62] H. Le, P. Moin and J. Kim, J. Fluid Mech. 330 (1997) 349-374.

[63] M. Lee and W.C. Reynolds, Bifurcating and blooming jets at high Reynolds number, in Fifth Symp. on Turbulent Shear Flows (Ithaca, New York, 1985) pp. 1.71.12 .

[64] S.K. Lele, J. Comput. Phys. 103 (1992) 16-42.

[65] A. Leonard, Adv. Geophys. A 18 (1974) 237-248.

[66] D.C. Leslie and G.L. Quarini, J. Fluid Mech. 91 (1979) 65-91.

[67] M. Lesieur, Turbulence in Fluids, Third Revised and Enlarged Edition (Kluwer Academic Publishers, Dordrecht, 1997).

[68] M. Lesieur and P. Comte, Large-eddy simulations of compressible turbulent flows, AGARD-VKI course Turbulence in compressible flows (Belgique, 2-5 June and USA 20-24 October, AGARD report 819, 1997) ISBN 92-836-1057-1.

[69] M. Lesieur and O. Métais, Annu. Rev. Fluid Mech. 28 (1996) 45-82.

[70] M. Lesieur, O. Métais and E. Garnier, J. Turbulence 1 (2000) 002.

[71] M. Lesieur and R. Rogallo, Phys. Fluids A 1 (1989) 718-722.

[72] M. Lesieur and D. Schertzer, J. Mécanique 17 (1978) 609-646.

[73] M. Lesieur, S. Yanase and O. Métais, Phys. Fluids A 3 (1991) 403-407.

[74] D. Liepmann and M. Gharib, J. Fluid Mech. 245 (1992) 643-668.

[75] D.K. Lilly, in Lecture Notes on Turbulence, edited by J.R. Herring and J.C. McWilliams (World Scientific, 1987) pp. 171-218.

[76] D.K. Lilly, Phys. Fluids A 4 (1992) 633-635. 
[77] S. Liu, C. Meneveau and J. Katz, J. Fluid Mech. 275 (1994) 83-119.

[78] O. Métais, C. Flores, S. Yanase, J.J. Riley and M. Lesieur, J. Fluid Mech. 293 (1995) 41-80.

[79] O. Métais and M. Lesieur, J. Atmos. Sci. 43 (1986) 857-870.

[80] O. Métais and M. Lesieur, J. Fluid Mech. 239 (1992) 157-194.

[81] O. Métais, M. Lesieur and P. Comte, Large-eddy simulations of incompressible and compressible turbulence, in Transition, Turbulence and Combustion Modelling, edited by A. Hanifi et al., ERCOFTAC Series (Kluwer Academic Publishers, 1999) pp. 349-419.

[82] C. Meneveau and J. Katz, Annu. Rev. Fluid Mech. 32 (2000) 1-32.

[83] A. Michalke and G. Hermann, J. Fluid Mech. 114 (1982) 343-359.

[84] Y. Miyake and T. Kajishima, Bull. JSME 29 (1986) 3347-3351.

[85] P. Moin and J. Kim, J. Fluid Mech. 118 (1982) 341-377.

[86] K. Nakabayashi and O. Kitoh, J. Fluid Mech. 315 (1996) 1-29.

[87] X. Normand, Transition à la turbulence dans les écoulements cisaillés libres et pariétaux, Ph.D. Thesis (National Polytechnic Institute, Grenoble, 1990).

[88] X. Normand and M. Lesieur, Theor. and Comp. Fluid Dyn. 3 (1992) 231-252.

[89] S.A. Orszag, J. Fluid Mech. 41 (1970) 363-386.

[90] S.A. Orszag, Statistical theory of turbulence, in Fluid Dynamics 1973, Les Houches Summer School of Theoretical Physics, edited by R. Balian and J.L. Peube (Gordon and Breach, 1977) pp. 237-374.

[91] J. Padilla-Barbosa and O. Métais, J. Turbulence 1 (2000) 009.

[92] R.A. Petersen, J. Fluid Mech. 89 (1978) 469-495.

[93] R.T. Pierrehumbert and S.E. Widnall, J. Fluid Mech. 114 (1982) 59-82.

[94] U. Piomelli, Phys. Fluids A 5 (1993) 1484-1490.

[95] U. Piomelli, W.H. Cabot, P. Moin and S. Lee, Phys. Fluids A 3 (1991) 1766-1771.

[96] U. Piomelli and J. Liu, Phys. Fluids A 7 (1995) 839-848.

[97] M. Rogers and R. Moser, Phys. Fluids A 6 (1994) 903.

[98] P. Sagaut, Introduction à la simulation des grandes échelles pour les écoulements de fluide incompressible (Springer-Verlag, 1998).

[99] M. Salinas-Vazquez, Simulations des grandes échelles des écoulements turbulents dans les canaux de refroidissement des moteurs fusée, Ph.D. Thesis (National Polytechnic Institute, Grenoble, 1999).

[100] M. Salinas-Vazquez and O. Métais, Large-eddy simulation of the turbulent flow in a heated square duct, in Direct and Large Simulation III, edited by P.R. Voke et al. (Kluwer Academic Publishers, 1999) pp. 13-24.

[101] G.S. Settles, T.J. Fitzpatrick and S.M. Bogdonoff, AIAA J. 17 (1979) 579-585.

[102] E.D. Siggia, J. Fluid Mech. 107 (1981) 375-406.

[103] C.B. da Silva, Ph.D. Thesis (National Polytechnic Institute, Grenoble, 2000).

[104] J.H. Silvestrini, Simulation des grandes échelles des zones de mélange : application à la propulsion solide des lanceurs spatiaux, Ph.D. Thesis (National Polytechnic Institute, Grenoble, 1996).

[105] J. Smagorinsky, Mon. Weath. Rev. 91 (1963) 99-164.

[106] P.R. Spalart, J. Fluid Mech. 187 (1988) 61-98.

[107] D.K. Tafti and S.P. Vanka, Phys. Fluids A 3 (1991) 642-656.

[108] G. Urbin, Étude numérique par simulation des grandes échelles de la transition à la turbulence dans les jets, Ph.D. Thesis (National Polytechnic Institute, Grenoble, 1998). 
[109] G. Urbin and O. Métais, Large-eddy simulation of three-dimensional spatiallydeveloping round jets, in Direct and Large-Eddy Simulation II, edited by J.P. Chollet, L. Kleiser and P.R. Voke (Kluwer Academic Publishers, 1997) pp. 35-46.

[110] G. Urbin, C. Brun and O. Métais, Large-eddy simulations of three-dimensional spatially evolving roud jets, in 11th symposium on Turbulent Shear Flows (Grenoble, September 8-11, 1997) pp. 25-23/25-28.

[111] A. Vincent and M. Meneguzzi, J. Fluid Mech. 258 (1994) 245-254.

[112] S. Yanase, C. Flores, O. Métais and J.J. Riley, Phys. Fluids A 5 (1993) 2725-2737. 